\title{
NEUROPLASTICITY: INDUCTION AND MODULATION BY EXTERNAL STIMULATION AND \\ PHARMACOLOGICAL INTERVENTION
}

\author{
Dissertation \\ zur Erlangung des Doktorgrades \\ der Mathematisch-Naturwissenschaftlichen Fakultäten \\ der Georg-August Universität zu Göttingen
}

vorgelegt von

MD., M.Sc. (Medical Neurosciences)

Min-Fang Kuo

aus Tainan

Göttingen 2007 
D 7

Referent: $\quad$ Prof. Dr. med. Walter Paulus

Korreferent: Prof. Dr. Stefan Treue

Prof. Dr. Thomas Rammsayer

Tag der mündlichen Prüfung: 06.07.2007 


\section{Acknowledgements}

I would like to express deep gratitude to the people providing their support during my doctoral studies. This thesis would not have been possible without tremendous support and guidance of Prof. Walter Paulus. As a mentor he offered me numerous opportunities and countless support during these years. It was his consistently innovative vision that led to the construction and execution of this work. I sincerely thank my supervisor Dr. Michael A. Nitsche for the gracious, patient guidance of my research, sharing his expertise and introducing me many exciting ideas. I would also like to address my thanks to Prof. Stefan Treue and Prof. Thomas Rammsayer, both members of my PhD committee, for the support during my study. My special gratitude goes to Prof. John Rothwell, whose constructive advice and supervision during my stay in London was influential on a great part of this work.

I give my thanks to Holger Rothkegel from whom I received a lot of help and who I shared many inspiring discussions with. A particular note of appreciation goes to Marion Kurze and Christine Crozier for their kind help so that I survived all the administrative procedures. I am also grateful to Peter Wenig for providing expert knowledge and support about the experiment settings. Besides, it was the fruitful discussion with Diane Ruge and James Teo during my London stay which contributed greatly to the quality of this thesis.

And here I cannot express sufficient gratitude to my family, so far away from me and yet the support from them is never less.

Finally, very special acknowledgement must be given to Thomas Hartmann for providing his invaluable assistance and endless patience during the course of the years. He has been a constant source of thoughtful feedback which keeps me grounded and encourages me to leap into the unknown. 


\section{Table of Contents}

Chapter 1 Introduction............................................................................1

1.1. Plasticity in central nervous system ................................................... 2

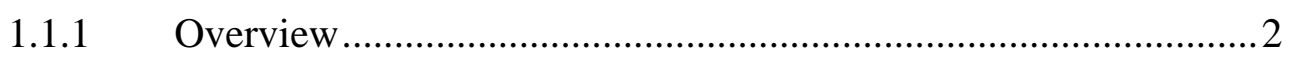

1.1.2 Motor cortex plasticity ............................................................ 3

1.2 Non-invasive brain stimulation in humans .......................................... 4

1.2.1 Transcranial magnetic stimulation ........................................... 4

1.2.2 Transcranial direct current stimulation ..................................... 5

1.2.3 Paired associative stimulation................................................. 6

1.3 Pharmacological modulation of human cortical plasticity........................ 7

1.3.1 Dopaminergic modulation of neuroplasticity .............................. 7

1.3.2 Cholinergic modulation of neuroplasticity ................................. 8

$1.4 \quad$ Neuroplasticity and motor learning …............................................... 9

Chapter 2 Original articles and manuscripts ................................................ 11

2.1 Sex differences in cortical neuroplasticity in humans ............................12

2.2 Boosting focally-induced brain plasticity by dopamine .......................... 18

2.3 Focusing effect of acetylcholine on neuroplasticity in the human motor

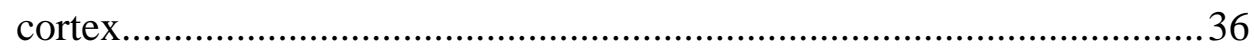

2.4 Timing-dependent modulation of associative plasticity by general network excitability in the human motor cortex .......................................55

2.5 No Major influence of homeostatic plasticity in complex motor learning

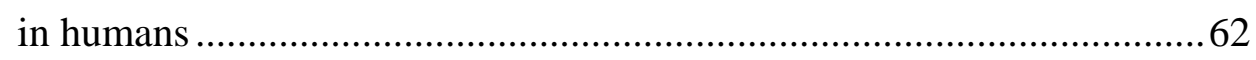

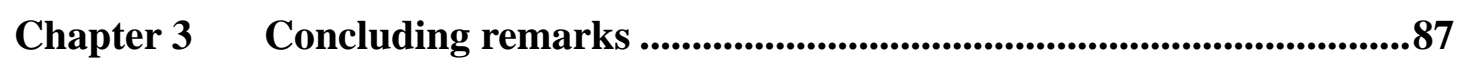

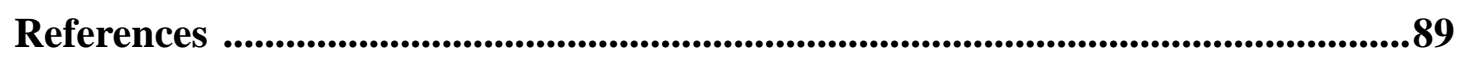

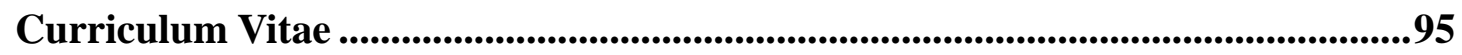




\section{Chapter 1}

\section{Introduction}

It is one of the most important capacities of the human brain to reorganize itself structurally and/or functionally in order to cope with the tremendous inputs from the surrounding world. This capacity, termed as neuroplasticity, has been intensively studied in modern neuroscience research, not only because it is considered the mechanism underlying several cognitive processes, but also because of its potential therapeutic application on neuropsychiatric disorders. The investigation of neuroplasticity was initially only possible on animal preparations. The recent development of advanced technologies now enables the non-invasive exploration of neuroplasticity directly on live brains, including humans. For example, transcranial magnetic stimulation (TMS) and transcranial direct current stimulation (tDCS) both serve as tools to induce cortical plasticity safely in humans, and therefore offer great opportunity for the better understanding of this phenomenon via direct induction or manipulation.

The present work contains three major parts: first, the basic concepts and factors which influence the induction and maintenance of human cortical plasticity are discussed. Here we explored a gender difference of neuroplasticity induced by tDCS in humans. The results demonstrate more facilitatory and less inhibitory neuroplasticity in males as compared to females. This might explain the different incidence of certain neurological diseases between females and males. The second part represents the modulation of cortical plasticity with pharmacological interventions. Dopamine (DA) and acetylcholine (ACh) represent two major neuromodulators of multiple brain functions. Several lines of evidence have suggested their involvement and multiple modes of action in neuroplasticity induction and modulation. Here the influence of DA and ACh on distinct forms of neuroplasticity, namely global and specific synaptic plasticity induced by tDCS and paired associative stimulation (PAS) respectively, are investigated in humans. We 
find that both DA and ACh enhance focal and suppress global excitability-enhancing plasticity. Thus it supports the hypothesis that DA and ACh focus specific synaptic inputs in neural networks in order to raise signal-to-noise ratio and thereby enhance information processing. The third part of the thesis focuses on the interaction between neuroplasticity modulation and learning processes. Homeostatic mechanisms are proposed to prevent neural networks from over-enhanced cortical excitability induced by positive-feedback Hebbian plasticity. Homeostatic plasticity can be achieved by global processes that regulate overall levels of neuronal and network activity within the range which is optimal for learning and memory. To study the regulation of homeostatic plasticity and its impact on cognitive functions, we first demonstrate the homeostatic plasticity generated by the combination of tDCS and PAS, with the former modulating global excitability and the latter inducing focal plasticity which has been suggested to represent the neurophysiological basis of learning. To explore whether the specific homeostatic mechanisms demonstrated in this study apply to learning processes, we further performed an experiment in which we combined tDCS with a motor learning task. The results of this experiment show that the specific impact of tDCS on motor learning is not in accordance with the homeostatic phenomenon observed neurophysiologically, indicating that cognitive functions are regulated in more complicated manners than simple homeostatic mechanisms.

The aim of this thesis is to elucidate the mechanisms for the induction and modulation of neuroplasticity in humans by external brain stimulation and pharmacological intervention as well as the implications in a functional and cognitive respect. This chapter will give a brief introduction to the background. Chapter 2 represents the main component of the thesis, and consists of published or submitted manuscripts of which the specific objectives will be briefly introduced beforehand. Finally, the last chapter gives a summary of the present work and conclusions with a prospective outlook and future directions of research.

\subsection{Plasticity in central nervous system}

\subsubsection{Overview}

Plasticity is an intrinsic property of the nervous system allowing short-term to long-lasting remodeling of neurosynaptic maps to adapt itself to the changing environment, and to optimize the functioning of brain networks. On the microscopic level, it is described as the dynamic modulation of synaptic strength including structural modifications of an increase in the size or number 
of synapses as well as functional alteration of synaptic transmission in response to the coincident activity of pre- and post-synaptic elements. Long-term potentiation and depression (LTP and LTD) represent a durable increase or decrease of synaptic plasticity involving several molecular regulating processes such as the expression of glutamatergic receptors (Malenka and Bear, 2004), and have been studied extensively across various species. In particular, associative forms of LTP and LTD have been the focus of plasticity research (Cooke and Bliss, 2006). The long-term modification of synaptic plasticity characterized by longevity, input specificity and associativity was linked to undermine the mechanisms of learning dynamics and memory storage (Martin et al., 2000) according to the rules of Hebbian plasticity stating that learning and memory are based on modifications of synaptic strength among neurons that are simultaneously active due to task repetition. Moreover, these mechanisms are important for the adaptive reorganization of cortical networks of the brain following physiological or pathological changes (Buonomano and Merzenich, 1998).

\subsubsection{Motor cortex plasticity}

Although synaptic plasticity has first been demonstrated in the hippocampus, the induction of both short-term and long-term plasticity in the form of changes in synaptic strengths is also well documented in the motor cortex (Hess and Donoghue, 1994; Rioult-Pedotti et al., 2000; Sanes and Donoghue, 2000). The horizontal inter-neuron connections within the primary motor cortex (M1) are suggested as the candidate substrate for M1 plasticity since these collateral connections functionally associate M1 neurons to form neuronal assemblies which construct dynamic motor maps and have a capacity for long-lasting synaptic modification (for review see (Sanes and Donoghue, 2000). M1 plasticity has been shown not only in animal experiments, but also in humans as revealed by neuroimaging and electrophysiology techniques (Hallett, 2001; Pascual-Leone et al., 2005). Plasticity in M1 has been shown to be functionally important. It guarantees a dynamic reorganization of the brain, e.g. following brain damage to compensate the function losses of brain networks (Nudo et al., 1996). Motor cortical representations can reorganize rapidly in response to different pathological forms of damage, and this capacity has drawn great attention and interest especially in restorative neurology (Butefisch, 2006; Duffau, 2006). The dynamic remodeling and synaptic modification of M1 circuits also play an essential role during motor learning and memory 
consolidation (Sanes and Donoghue, 2000; Muellbacher et al., 2002; Doyon and Benali, 2005).

It is the purpose of the present work to improve knowledge of these phenomena in order to guide cortical plastic potentials for the functional recovery after brain damage, and to better understand the learning and memory processes associated with the motor cortex.

\subsection{Non-invasive brain stimulation in humans}

Although it is attractive to link neuroplasticity to cognition on the macroscopic level to learn more about the neuronal basis of learning and memory, the direct demonstration of such long-term plastic changes in humans has not been possible until recently when functional neuroimaging and non-invasive brain stimulation techniques were better developed. Functional magnetic resonance imaging (fMRI), positron emission tomography (PET), and Electrico-/magnetoencephalography (EEG/MEG) enable the elucidation of the spatial change and temporal resolution of brain activation patterns due to cortical reorganization or cognitive processing. On the other hand, the interventional procedures can further increase the understanding of cortical plasticity via active modulations. In the past decades technical advances in the field of external brain stimulations, including transcranial magnetic stimulation (TMS) and transcranial direct current stimulation (tDCS), have been developed for this purpose.

\subsubsection{Transcranial magnetic stimulation}

TMS is a valuable tool in modern neurophysiology research because it can be applied to monitor various aspects of cortical excitability with different paradigms, and to induce 'virtual lesions' by disturbing the brain function of certain areas (Pascual-Leone et al., 2000; Terao and Ugawa, 2002; Rossini and Rossi, 2007). Originally introduced to noninvasively investigate nervous propagation along the corticospinal tract, spinal roots, and peripheral nerves in humans, TMS is nowadays extensively used in basic and clinical neurophysiology (Pascual-Leone, 2002). Principally it generates an electric current in neuronal tissue without the need for direct physical contact between the stimulation coil and the brain, thereby activating the representational cortical area. In the motor cortex, motor evoked potentials of the respective 
muscle, of which the cortical representation area is stimulated, can be recorded and analyzed by single-pulse TMS (Rothwell, 1993), thus it allows motor output to be mapped precisely to a given body district following interventions such as plasticity induction procedures. Moreover, special TMS paradigms including paired-pulse TMS, input-output (IO) curve, and I-waves can be used to specify and to localize the cortical sub-systems involved in changes of cortical excitability (Ziemann and Rothwell, 2000; Chen, 2004). It is therefore suitable for providing information about the pathophysiology of the brain in various neuropsychiatric diseases as well as on the mechanisms of brain plasticity. Additionally, neuroplasticity can be elicited either by repetitive TMS applied with certain frequency (Fitzgerald et al., 2006) or by coupling TMS with peripheral sensory input, termed as rTMS and paired associative stimulation (PAS, see section 1.2.3) respectively. Both phasic stimulation protocols are intensively applied in basic and clinical neurophysiology to investigate and modulate cortical plasticity.

\subsubsection{Transcranial direct current stimulation}

The application of weak current to change neuronal excitability was first introduced in the 1960's in animals (Bindman et al., 1964). Direct currents were delivered by intracerebral or epidural electrodes in anesthetized rats, and induced activity and excitability diminutions or enhancements of the sensorimotor cortex which can be stable for hours after the stimulation. It was suggested that the long-lasting excitability changes shared some features with long term synaptic plasticity (Nitsche et al., 2003b). For example, these effects were protein synthesis-dependent (Gartside, 1968) and accompanied by modifications of intracellular cAMP- and calcium-levels (Hattori et al., 1990; Islam et al., 1995). Later the direct current stimulation was applied transcranially to human subjects, mainly psychiatry patients, with effective results such as anodal stimulation diminishing depressive symptoms (Costain et al., 1964), while cathodal stimulation reduced manic symptoms (Carney, 1969). However, the positive effect was not reproducible in some studies and such discrepancy might have been due to the difficulty of the objective evaluation of the effects during these times, and the various parameters applied in different labs. The technique was therefore nearly forgotten until recently when the non-invasive tools including neuroimaging and transcranial stimulations were rapidly developed allowing the monitoring of brain activity and excitability changes. The re-evaluation of tDCS showed promising results of inducing acute 
and long-lasting cortical excitability changes. The effects depend on the direction of the current flow, which is defined generally by the electrode positions and polarity: anodal stimulation enhances excitability, while cathodal stimulation decreases it (Nitsche and Paulus, 2000, 2001; Nitsche et al., 2003a). The plastic changes can be blocked by co-administrating the $N$-methyl-D-aspartic acid (NMDA) receptor antagonist (Liebetanz et al., 2002; Nitsche et al., 2003d), which indicates at the involvement of synaptic mechanism other than membrane polarization in the after-effects (Purpura and McMurtry, 1965) underlining the induction of LTP/LTD-like plasticity by tDCS. Conceivably, tDCS can be used as a method for tonic induction and modulation of neuroplasticity in humans since it elicits reversible, long-lasting cortical excitability changes reliably and non-invasively. In addition to the motor cortex which had been studied more intensively initially, tDCS can also exert effective modification of somatosensory, visual systems (Matsunaga et al., 2004; Antal et al., 2006), and modulation of cognition in the prefrontal cortex with a combination of specific psychophysics protocols (Nitsche et al., 2003c; Antal et al., 2004; Kincses et al., 2004; Fregni et al., 2005).

\subsubsection{Paired associative stimulation}

In PAS, repetitive low-frequency peripheral nerve stimulation is combined with TMS over the contralateral motor cortex. This associative stimulation has been shown to induce plastic changes of excitability in the human motor cortex (Stefan et al., 2000). Its principles of design were shaped related to associative LTP which is induced by correlated input-output stimulation protocols in animal experiments, a cellular mechanism likely to be relevant for learning and memory. The direction of PAS-induced plastic changes is determined by the inter-stimulus interval. Cortical excitability is enhanced when the single-pulse TMS is preceded by the sensory input into the motor cortex, whereas the opposite causes excitability diminution (Wolters et al., 2003). Thus PAS shares some features with spike-timing-dependent synaptic plasticity. This kind of plasticity is well documented in animal studies as well as computational modeling, and is considered to play an important role in information processing and storage within neural networks (Dan and Poo, 2004). The neuroplasticity induced by PAS is diminished by blocking NMDA receptors (Stefan et al., 2002), which is also consistent with the involvement of LTP/LTD-like processes. Compared to the generalized tDCS-induced excitability changes, PAS induces cortical plasticity via more specific synaptic modifications. This is 
of particular importance as the application of various methods to induce distinct forms of neuroplasticity will provide better insight into cognition-related cortical network plasticity.

Taken together, the aforementioned technologies are well-developed to investigate brain plasticity in humans non-invasively and painlessly. This serves as the major methodology of the present work, in which neuroplasticity in the human motor cortex as a model system is investigated with the application of tDCS and PAS by using TMS-induced MEPs as index of resultant changes in motor cortical excitability.

\subsection{Pharmacological modulation of human cortical plasticity}

A large number of experimental studies have shown that exogenous manipulation of central neurotransmitter levels can directly affect plastic changes in the brain and can modulate the effects of experience and training (Gu, 2002). Dopamine and acetylcholine are applied as clinical pharmacotherapy in various neuropsychiatry disorders associated with a maladaptation of brain plasticity, despite the direct evidence in humans supporting its physiological mechanism is still not completely understood. Given the involvement of both transmitters in neuroplasticity, it is of great interest how pharmacological intervention will influence the neurophysiologically induced plasticity.

\subsubsection{Dopaminergic modulation of neuroplasticity}

It is well recognized that DA plays a critical role in normal cognitive process and neuropsychiatric pathologies of the central nervous system. On the cellular level, DA has been demonstrated to exert both facilitation and inhibition of neuronal excitability or synaptic activity (Seamans and Yang, 2004). The distinct features of DA modulation on the cognitive level are also observed in both animal and human experiments (Jay, 2003).

Both the improvement and deterioration of cognitive function such as working memory were reported with application of either agonist or antagonist of DA receptors (Jay, 2003). Clinically, DAergic therapy on neurological or psychiatric diseases occasionally encounters limitations such as unexpected 
adverse effect or inconsistent outcome, which is probably associated with the heterogeneous pattern of DA action described above (Cools, 2006). This indicates DA might exert multiple functions according to external challenge and environmental demand as well as internal neural network status on the macroscopic level, which hampers the step to bridge microscopic findings with the behavioral phenomena of DAergic modulation. It is suggested that the systemic, complex excitability modifications generated by DA result in a focusing effect on neuroplasticity and related cognitive functions (Seamans and Yang, 2004). This could explain that DA has an antagonistic influence on the performance depending on the task demands, i.e. flexibility or focusing maintenance of input representation during information processing. However, the neurophysiological evidence, especially in humans, for such a focusing effect is still lacking so far.

\subsubsection{Cholinergic modulation of neuroplasticity}

The cholinergic system is implicated in functional, behavioural and pathological states including cognitive function, nicotine addiction, Alzheimer's disease, Tourette's syndrome, epilepsies and schizophrenia. ACh would be expected to facilitate the induction of plasticity since general evidence indicates ACh can produce long-lasting increases in neural responsiveness (Rasmusson, 2000). However, similar to DA, the understanding of cholinergic modulation is complex because it is the result of a mixture of positive and negative modulation, implying that there are various modes of cholinergic regulation in cortical functions (Lucas-Meunier et al., 2003). Enhancing cholinergic level in the brain is the main target of current pharmacotherapy for neurocognitive deficits aiming at restoring cognitive function or at least preventing its decline. It is generally accepted that ACh increases signal-to-noise ration probably via enhancing the strength of afferent input relative to feedback and activating intrinsic mechanisms for persistent spiking as well as increasing the modification of synapses (Hasselmo and Giocomo, 2006). These effects might enhance specific synaptic plasticity within neural networks thereby contributing to the learning process and memory encoding. Similar to DA, the direct demonstration in humans of cholinergic focusing function on the neurophysiological level is missing.

It is possible to study the neurophysiological mechanisms of DA and ACh modification in plasticity-related cortical circuitry with the application of 
external brain stimulation such as tDCS and PAS in healthy human subjects (Nitsche et al., 2006). Moreover, this approach will provide more information about how these neurotransmitters modulate discrete forms of neuroplasticity and thus lead to further implication of cognitive processes in human brain.

\subsection{Neuroplasticity and motor learning}

Learning refers to the process by which a relatively lasting change in potential behavior occurs as a result of practice or experience. Neurophysiologically it involves the constant adaptation of the central nervous system to incoming information in order to optimize behavioural outcome. This requires the dynamic, plastic reorganization of neural connectivity in cortical networks. M1 is suggested to play an important role in forming new or adapting existing motor skills, as exemplified by recent works in experimental animals including primates and humans (Gandolfo et al., 2000; Sanes and Donoghue, 2000; Li et al., 2001; Ilic and Ziemann, 2005). The remarkable flexibility and reorganization of motor cortical representations within M1 indicate an important contribution of plastic changes to motor learning. However, uncontrolled learning-associated neuroplasticity will drive the brain to a deleterious state of saturated up- or down-regulation of neural network connections. To prevent such instability caused by positive-feedback and correlation-based plasticity, homeostatic regulatory mechanisms were therefore introduced. Metaplasticity, also referred to as homeostatic plasticity and initially studied in neuronal network simulations and at the synaptic level, encompasses regulatory processes that maintain the stability and functionality of neural networks subject to Hebbian plastic modifications according to their history of use and the average activity level (Abraham and Bear, 1996; Turrigiano and Nelson, 2000). Recently this was also demonstrated in human subjects on the neurophysiological level (Lang et al., 2004; Siebner et al., 2004; Ziemann et al., 2004; Stefan et al., 2006). It was demonstrated that enhancing or diminishing motor cortex excitability with tDCS resulted in inversely directed effects on excitability of rTMS protocols which are ineffective to induce any excitability modifications if administered alone (Lang et al., 2004; Siebner et al., 2004). Moreover, preceding learning processes were shown to reverse LTD-like plasticity induced by inhibitory PAS and prevent LTP-like plasticity by facilitatory PAS (Ziemann et al., 2004; Stefan et al., 2006). Such regulating mechanism may keep the synaptic strengths and cortical excitability within a dynamic range that is optimal for learning process. However, 
experimental evidence for the significance of homeostatic plasticity in learning is still lacking.

As the focal plasticity induced by PAS is postulated to mimic learning processes while tDCS could be used to modify the global background neural excitability, it is thus plausible to elucidate the mechanisms and functional significance of metaplastic regulation of cortical plasticity associated with motor learning in healthy human subjects with both external brain stimulation protocols. Combining tDCS with a motor learning paradigm might in a second step be able to test the transferability of the neurophysiologically achieved results to the behavioural level.

In summary, the main theme of this work is to elucidate the basic mechanisms of neuroplasticity on both neurophysiological and behavioral levels with well-developed external brain stimulation techniques and pharmacological interventions. The knowledge we gain here might further help to optimize the manipulation of cortical plasticity for clinical therapeutic interventions. 


\section{Chapter 2}

\section{Original articles and manuscripts}

This chapter includes published articles and also manuscripts in submission.

- Kuo MF, Paulus W, Nitsche MA. Sex differences in cortical neuroplasticity in humans. Neuroreport. 2006 Nov 6;17(16):1703-7

- Kuo MF, Paulus W, Nitsche MA. Boosting focally-induced brain plasticity by dopamine. Cerebral Cortex (in press)

- Kuo MF, Fregni F, Paulus W, Nitsche MA. Focusing effect of acetylcholine on neuroplasticity in the human motor cortex. (in submission)

- Kuo MF, Unger M, Antal A, Liebetanz D, Lang N, Tergau F, Paulus W, Nitsche MA. No Major influence of homeostatic plasticity in complex motor learning in humans. (in submission)

- Nitsche MA, Roth A, Kuo MF, Fischer AK, Liebetanz D, Lang N, Tergau F, Paulus W. Timing-dependent modulation of associative plasticity by general network excitability in the human motor cortex. Journal of Neuroscience 2007 Apr 4;27(14):3807-12 


\subsection{Sex differences in cortical neuroplasticity in humans}

It is a well-known fact that brain functions differ gradually between males and females, as shown by certain aspects of cognitive performance (Kimura, 1999; Cahill, 2005) and the susceptibility to develop certain neurological diseases. However, the neuronal foundations underlying these differences are still not well understood. Our aim was to explore gender differences of neuroplasticity induced by tDCS in the human motor cortex. The data collected from previously conducted motor cortex tDCS studies was re-analyzed retrospectively. During a short DC stimulation, which elicits no after-effects, the female group showed more inhibition. Similarly, in women the excitability-diminishing after-effects of cathodal tDCS were significantly prolonged, as compared to the male group. In contrast, no relevant difference between male and female subjects was revealed for the results of excitability-enhancing anodal tDCS. This pattern of results suggests a gender effect of neuroplasticity induction, which could be responsible for the different disease prevalence such as dystonia or Parkinson's disease. 


\title{
Sex differences in cortical neuroplasticity in humans
}

\author{
Min-Fang Kuo, Walter Paulus and Michael A. Nitsche \\ Department of Clinical Neurophysiology, Georg-August-University, Goettingen, Germany \\ Correspondence and requests for reprints to Dr Min-Fang Kuo, Department Clinical Neurophysiology, Georg-August-University, Robert-Koch-Str. 40, \\ 37075 Goettingen, Germany \\ Tel: + 551 39957l; fax: + 551 398126; e-mail: i5484133@web.de
}

Received 3 July 2006; revised 26 July 2006; accepted 27 July 2006

\begin{abstract}
In the present study, we explore sex differences of neuroplasticity in humans, as revealed by transcranial direct current stimulation, which induces motor cortical excitability changes both during and after stimulation. We retrospectively re-analyzed data collected from previous transcranial direct current stimulation studies. In women, the excitability-diminishing after-effects of cathodal transcranial direct current stimulation were relevantly prolonged compared with the male group. Similarly, during a short direct current
\end{abstract}

Keywords: brain stimulation, gender, human, neuroplasticity stimulation that elicits no after-effects, the female group showed more inhibition. In contrast, no significant differences between male and female study participants were found for excitabilityenhancing anodal transcranial direct current stimulation. These results suggest sex differences, possibly due to the effects of sex hormones, in the modulation of human cortical plasticity. NeuroReport 17:1703-1707 (c) 2006 Lippincott Williams \& Wilkins.

\section{Introduction}

Neuroplasticity, a continuous process of change in neuronal activities involving brain functions such as cognition, can be modulated by gender effects. Sex differences of synaptic plasticity and cognitive functions in animal studies have been demonstrated [1-4], and sex hormones were suggested as modulating such effects [5-7]. In humans, the reports of gender differences are less numerous and mainly focus on cognitive performance, such as spatial skills or verbal tasks in which men or women tend to excel, respectively (for a review, see [8]). Clinical observations suggest gender differences of brain physiology as one plausible cause for the sex-dependent susceptibility to some neurological diseases such as dystonia or Parkinson's disease. These findings indicate possible differences in human brain function between the sexes with regard to neuroplasticity, both physiological and pathological, in spite of the methodological difficulties of directly investigating human cortical neurophysiology.

Since the discovery that continuous weak direct current stimulation induces lasting excitability changes in the human motor cortex $[9,10]$, transcranial direct current stimulation (tDCS) has been applied in various studies of induction and modulation of cortical neuroplasticity. While direct current (DC) stimulation for $4 \mathrm{~s}$ elicits brief excitability enhancements or reductions that do not outlast the stimulation itself, tDCS applied for some minutes has been demonstrated to induce long-lasting after-effects for more than $1 \mathrm{~h}$ after the end of stimulation $[10,11]$. Pharmacological studies show that antagonizing $N$-methyl-D-aspartate receptors abolishes tDCS-induced neuroplasticity [12,13], indicating that the possible underlying mechanisms share features with long-term potentiation and long-term depression. Therefore, tDCS is suitable as a noninvasive tool to study neuroplastic properties of the human cerebral cortex.

To explore the gender-specific modulation of neuroplasticity in humans, we re-analyzed the data from previous experiments in which tDCS was applied in healthy humans to study motor cortical functions. We reviewed genderdependent variations and differences in tDCS-induced, immediate and long-term effects on cortical plasticity, in order to shed light on the gender differences in human neurophysiological characteristics.

\section{Methods}

\section{Study participants}

We analyzed retrospectively the results from tDCS studies performed in our laboratory, in which 118 participants (66 women and 52 men) were included (see Table 1). The mean (SD) age of the male group was 27.4 (3.9) years, while that of the female group was 26.2 (2.2) years. In the studies of the intra-current excitability changes, data from 33 study participants (16 women and 17 men) were collected and analyzed. Eighty-five participants (47 women and 38 men) received $\mathrm{tDCS}$ to produce long-lasting after-effects. All of the participants were healthy, were taking no medication and had given written informed consent. The investigations were approved by the Ethics Committee of the University of Goettingen, and conformed to the Declaration of Helsinki. 
Table I Details of the different stimulation paradigms and participant characteristics of the experiments

\begin{tabular}{lcccc}
\hline & $\begin{array}{c}\text { DC } \\
\text { polarity }\end{array}$ & $\begin{array}{c}\text { DC } \\
\text { duration }\end{array}$ & $\begin{array}{c}\text { Number of } \\
\text { participants }\end{array}$ & $\begin{array}{c}\text { Age } \\
\text { (years } \pm \text { SD) }\end{array}$ \\
\hline Intra-DC effects & Anodal & $4 \mathrm{~s}$ & 16 female & $23.94 \pm 2.67$ \\
& Cathodal & & $\begin{array}{c}17 \text { male } \\
\text { Long-lasting after-effects }\end{array}$ & $\begin{array}{c}25.41 \pm 2.87 \\
\end{array}$ \\
& Anodal & 13 min & 40 female & $25.37 \pm 2.51$ \\
& Cathodal & 9 min & 45 female & $24.12 \pm 2.72$ \\
& & & 29 male & $25.85 \pm 2.69$ \\
\hline
\end{tabular}

In the intra-tDCS group, the DC stimulation lasted for $4 \mathrm{~s}$, and all the 33 participants had both anodal and cathodal tDCS. For the long-lasting tDCS, 62 participants (38 female, 24 male) took part in both anodal and cathodal stimulation. tDCS, transcranial direct current stimulation; DC, direct current.

\section{Transcranial direct current stimulation}

tDCS was applied with a specially developed, batterydriven constant current stimulator (Schneider Electronic, Gleichen, Germany; maximal output: $2 \mathrm{~mA}$ ) and was transferred with a saline-soaked pair of surface sponge electrodes $\left(35 \mathrm{~cm}^{2}\right)$. One of the electrodes was placed over the representational area of the right abductor digiti minimi muscle (ADM) as determined by transcranial magnetic stimulation (TMS), the other electrode above the right orbit. In the different experiments, the currents ran continuously for $4 \mathrm{~s}$ (intra-DC effects), or $9 \mathrm{~min}$ (cathodal stimulation) and 13 min (anodal stimulation) (long-lasting after-effects) with an intensity of $1 \mathrm{~mA}$.

\section{Measurement of motor cortical excitability \\ TMS-elicited motor evoked potentials (MEPs) were re- corded to measure excitability changes in the representa- tional motor cortical area of the right ADM. Single-pulse TMS was conducted either by a Magstim 200 magnetic stimulator (Magstim Company, Whiteland, Dyfed, UK) or a Magstim rapid-stimulator (Magstim Inc., Dyfed, UK), with a figure-of-eight magnetic coil (diameter of one wind- ing $=70 \mathrm{~mm}$, peak magnetic field $=2.2 \mathrm{~T}$ ). All of the intra- tDCS studies were performed with a Magstim 200, while the after-effects were obtained with either the Magstim 200 or the Magstim rapid-stimulators in the original studies. The coil was held tangentially to the skull, with the handle pointing backwards and laterally at $45^{\circ}$ from the midline. The optimal position was defined as the site where stimulation resulted consistently in the largest MEPs. A surface electromyogram was recorded from the right $\mathrm{ADM}$ with $\mathrm{Ag}-\mathrm{AgCl}$ electrodes in a belly-tendon montage. The signals were amplified and filtered with a time constant of $10 \mathrm{~ms}$ and a low-pass filter of $2.5 \mathrm{kHz}$, then digitized at an analogue-to-digital rate of $5 \mathrm{kHz}$ and further relayed into a laboratory computer using the Neuroscan software collec- tion (Neuroscan, Herndon, Virginia, USA).}

\section{Experimental procedures}

Both experiments (intra-tDCS and long-lasting after-effects) were conducted in a repeated measurement design. The participants were seated in a reclining chair. The left motor cortical representational area of the right ADM was identified by finding the TMS coil position eliciting the largest MEPs in the ADM. The intensity of stimulator output was adjusted for baseline recording so that the average stimulus produced an MEP of $\sim 1 \mathrm{mV}$. For the intra-current excitability changes, we recorded a randomized series $(0.1 \mathrm{~Hz})$ of 15 MEPs elicited by TMS (i) immediately before the end of a 4-s-long current stimulation or (ii) without preceding current stimulation. Both polarities of DC stimulation were carried out in one session in randomized order. For the long-lasting after-effects, we first recorded a baseline of TMS-evoked MEPs (20 stimuli) at $0.25 \mathrm{~Hz}$. The motor cortical DC stimulation electrode was then fixed above the left motor cortex and the other was fixed at the contralateral forehead position above the orbit. DC current was applied as 13-min anodal or 9-min cathodal stimulation, which had been demonstrated in previous studies to modify cortical excitability for approximately $1 \mathrm{~h}$ after the end of stimulation [10]. Immediately after tDCS, 20 MEPs were recorded every $5 \mathrm{~min}$ at $0.25 \mathrm{~Hz}$ for $30 \mathrm{~min}$, then 60 and $90 \mathrm{~min}$ after cessation of DC stimulation.

\section{Statistics}

An analysis of variance (ANOVA) model for repeated measures [independent variables: time course, gender, tDCS polarity and stimulator type (after-effects only)] was applied in both intra-tDCS and long-lasting after-effect conditions. MEP amplitude means were normalized as a quotient of the without-current (intra-DC condition) or pre-current (longlasting after-effect condition) baseline. For the intra-DC effects, gender comparisons were carried out separately for anodal and cathodal data using post-hoc Student's $t$-test (independent samples). Paired $t$-tests were performed to compare the effect of tDCS between baseline and poststimulation values. Student's $t$-tests for independent samples were used to determine the gender effect on MEP amplitude at each time point. The relationship between age and MEP changes was assessed by computing Pearson's correlation coefficient. All comparisons and correlations were two-tailed. Statistical significance was assumed at $P \leq 0.05$

\section{Results}

One hundred and eighteen individuals (66 women and 52 men) who participated in tDCS studies performed in our laboratory were included (see Table 1).

For the intra-effects of tDCS (4s tDCS, which produces no after-effects), the ANOVA revealed a significant effect of tDCS polarity (see Table 2). The inhibition of the MEP amplitude during cathodal stimulation was $0.786(\mathrm{SE}=0.02)$ in the female participants and $0.838(\mathrm{SE}=0.01)$ in the male participants. Student's $t$-tests revealed that the difference between the groups was significant $(P=0.02)$. MEP amplitudes did not differ between genders for the anodal tDCSinduced effect (men: 1.361 \pm 0.09 ; women: $1.347 \pm 0.09$; $P=0.91$ ) (Fig. 1).

For the neuroplastic after-effects of tDCS, the results of the ANOVAs show significant main effects of time course and tDCS polarity, and significant interactions of tDCS polarity $\times$ time course, gender $\times$ time course, stimulator type $\times$ time course, gender $\times$ stimulator type $\times$ time course, gender $\times$ tDCS polarity $\times$ time course and gender $\times$ stimu- 
Table 2 Results of ANOVAs

\begin{tabular}{|c|c|c|c|}
\hline & d.f. & $\mathrm{F}$ & $P$ \\
\hline \multicolumn{4}{|l|}{ Intra-tDCS } \\
\hline tDCS (polarity) & I & 76.451 & $<0.001^{*}$ \\
\hline Gender & I & 0.300 & 0.588 \\
\hline Gender $\times$ polarity & I & 0.094 & 0.761 \\
\hline \multicolumn{4}{|l|}{ Long-term after-effects of tDCS } \\
\hline Time course & 9 & 2.420 & $0.001^{*}$ \\
\hline Gender & I & 1.437 & 0.233 \\
\hline TMS stimulator type (stimulator) & I & 0.472 & 0.493 \\
\hline tDCS polarity & I & 151.938 & $<0.001^{*}$ \\
\hline Time $\times$ gender & 9 & 2.947 & $0.002^{*}$ \\
\hline Time $\times$ stimulator & 9 & 3.469 & $<0.001^{*}$ \\
\hline Time $\times$ polarity & 9 & 28.588 & $<0.001^{*}$ \\
\hline Time $\times$ gender $\times$ stimulator & 9 & 2.063 & $0.030^{*}$ \\
\hline Time $\times$ gender $\times$ polarity & 9 & 2.058 & $0.030^{*}$ \\
\hline Time $\times$ stimulator $\times$ polarity & 9 & 1.242 & 0.265 \\
\hline Time $\times$ gender $\times$ stimulator $\times$ polarity & 9 & 1.931 & $0.044^{*}$ \\
\hline
\end{tabular}

Repeated-measures ANOVAs were calculated for the intra-tDCS and the long-lasting after-effects. Polarity refers to anodal and cathodal stimulation. Asterisks indicate significant results $(P<0.05)$. ANOVAs, analyses of variance; tDCS, transcranial direct current stimulation; $D C$, direct current.

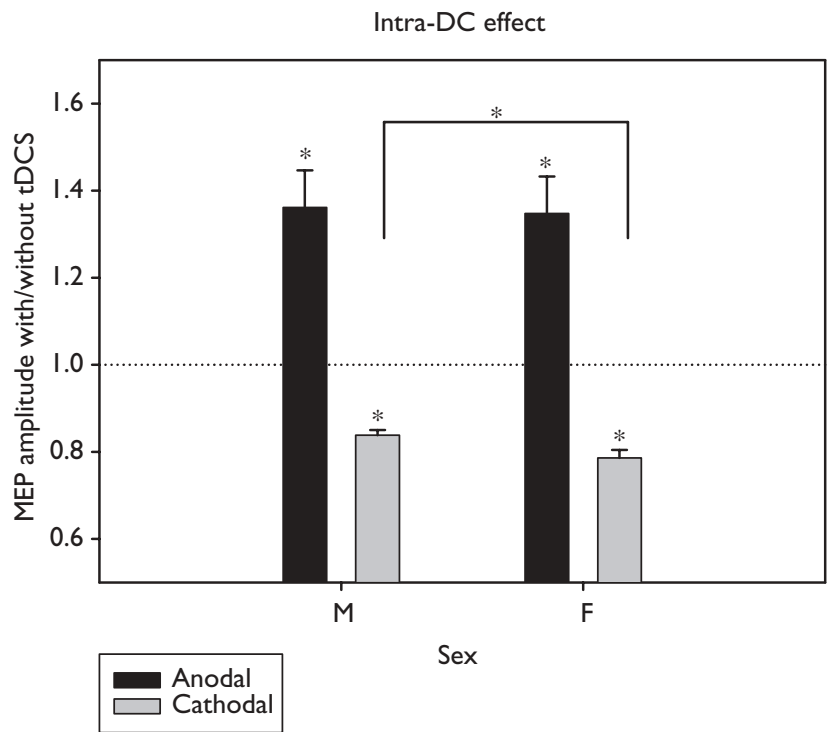

Fig. I Cortical excitability shifts during transcranial direct current stimulation (tDCS) in male and female groups. As the results show, the reduction of cathodal tDCS-generated cortical excitability is greater in female participants. No gender difference is observed in the enhancement of motor evoked potential size with regard to anodal stimulation. Asterisks indicate significant deviations of the current from the noncurrent conditions and differences between male and female groups in the cathodal tDCS condition. Error bars indicate SEM. M, male; F, female.

lator type $\times$ tDCS polarity (see Table 2 ). As demonstrated by the results of the post-hoc $t$-tests, the reduction of MEP amplitude after cathodal tDCS was significant for $30 \mathrm{~min}$ after tDCS in the male group and for $60 \mathrm{~min}$ in the female one. Even $90 \mathrm{~min}$ after $\mathrm{tDCS}$, a non-significant trend for inhibition was observed in the female group. Inhibition thus lasted significantly longer in the female group than in the male group (Fig. 2). The inter-gender difference was significant at the time points of $0(P=0.013), 10(P=0.043)$,

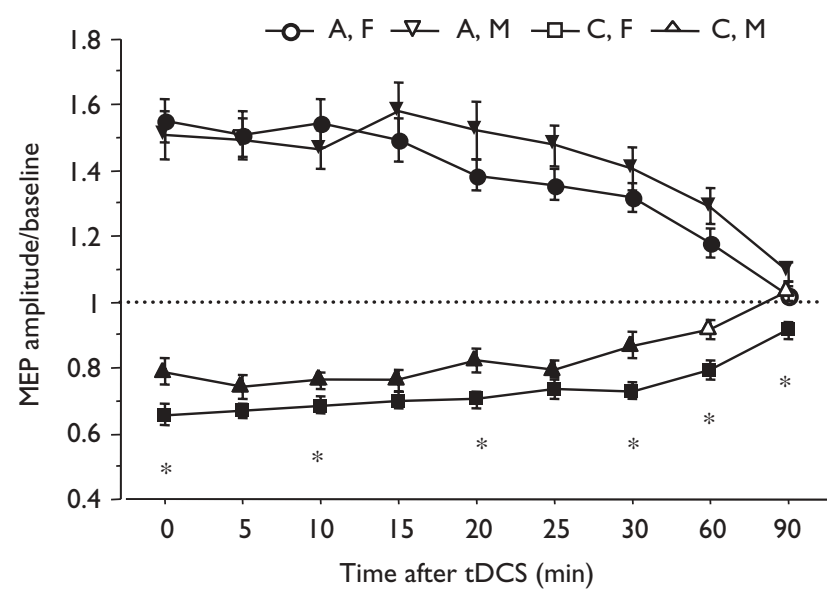

Fig. 2 Gender differences in transcranial direct current stimulation (tDCS)-induced long-lasting after-effects on cortical excitability. Prolonged inhibition caused by cathodal stimulation in the female group is shown at 60 and $90 \mathrm{~min}$ after termination of direct current, while in the male group the motor evoked potential sizes returned to baseline at these time points. Asterisks indicate significant differences between genders regarding identical current conditions and time points; filled symbols represent significant deviations from the respective baseline within each gender/tDCS combination. Error bars indicate SEM. A, anodal stimulation; C, cathodal stimulation; $M$, male; F, female.

$20 \quad(P=0.008), \quad 30 \quad(P=0.003), \quad 60 \quad(P=0.009) \quad$ and 90 $(P=0.004) \mathrm{min}$ after cathodal stimulation (Student's $t$-test, two-tailed). No significant gender difference in excitability enhancement induced by anodal tDCS was found in either time point. The increase of MEP amplitude, however, remained significant for male participants $90 \mathrm{~min}$ after tDCS, while it returned to baseline in the female group at this time point. Concerning the possible effects of age, no correlation with age was found for the aftereffects of both anodal and cathodal stimulation when the correlation between MEP ratio and age was analyzed for each time point (correlation coefficients: -0.224-0.144; $P=0.070-0.984)$. Therefore, the influence of age cannot explain the gender differences in tDCS-induced neuroplasticity.

\section{Discussion}

To the best of our knowledge, the results of this study present direct evidence for the first time of gender differences in neuroplasticity in the human motor cortex. Specifically, excitability-diminishing neuroplasticity was prolonged and enhanced in female participants, as compared with male participants. This effect was prominent for long-lasting plasticity, but also observable during shortlasting tDCS. Interestingly, it was specific for the tDCSinduced excitability diminution, as the effects of excitabilityenhancing anodal tDCS on MEP amplitudes did not differ between sexes.

The greater reduction of the MEP amplitude during cathodal tDCS in the female participants could be related to the effects of sex hormones such as progesterone, which contributes to the reduction of cortical excitability in women with normal menstrual cycles. Using the paired-pulse TMS paradigm, Smith et al. $[14,15]$ demonstrated a tendency to 
produce less intracortical inhibition and more facilitation when the circulating estradiol was higher, while the reverse effect was observed in the luteal phase, with a higher progesterone level, relative to the mid-follicular phase, and the similar effects were also observed in the TMS-induced silent period [16]. Moreover, insufficient levels of progesterone are related to premenstrual seizure exacerbations, and the inhibitory effect on cortical excitability can be restored with treatment of progesterone supplementation [17]. The underlying mechanisms are suggested to be associated with tonic inhibition mediated by $\gamma$-aminobutyric acid type $A$ receptors [18]. Thus, progesterone may serve as one of the candidates to modulate the inhibitory neuronal circuits in the motor cortex. It may be responsible for a stronger inhibition during cathodal tDCS. By increasing the effect of cathodal tDCS on membrane polarization during stimulation - which is a pre-requisite for the subsequent evolvement of after-effects - progesterone may have also favored the induction of neuroplastic excitability diminutions caused by prolonged cathodal tDCS.

In contrast to the lack of a gender-specific effect on anodal tDCS-elicited excitability enhancements, Inghilleri et al. [19] report that high estrogen levels in the late follicular phase increase the MEP size after fast, excitability-enhancing repetitive TMS, whereas during the period with lower estrogen levels such enhancement was diminished when compared with a male group. Some studies also demonstrated that long-term potentiation is easier to induce in male than in female rats $[20,21]$. It is possible that in the present study these respective gender differences were masked by non-controlled hormone levels in female participants. Further, prospective investigations are needed to elucidate the specific role of ovarian hormones in cortical neuroplasticity during the hormone cycle.

With regard to other parameters, which might have influenced the results, ANOVA shows that the type of TMS stimulator used to record MEPs influences the time course of recorded neuroplasticity. Owing to the results of ANOVA, this effect is independent of the gender effect on neuroplasticity (significant interaction between time course, tDCS polarity and gender). As it is known that the current wave form elicited by the two types of TMS stimulator used in this study (monophasic vs. biphasic) might affect, at least in part, different neuronal populations [22], this might be a hint that tDCS induces neuroplasticity in specific neuronal populations. The investigators performing the experiments and the individuals participating in the respective experiments, however, co-varied systematically with the type of TMS stimulator used. Studies should be performed in which these parameters are controlled to explore this phenomenon to a greater extent.

Although age differed slightly between the male and female groups, it fails to explain gender difference in neuroplasticity as the correlation analysis showed no significance.

\section{Conclusion}

Research on gender differences has long been of great interest. A surge of studies that highlight the influence of gender on many areas of cognition and behavior has appeared over the past decades [23]. The present findings indicate that inhibitory neuroplastic effects are more pronounced in the female participants. This may at least partially explain the predominance of the male patients in some movement disorders that are associated with abnormal excitability or reduced inhibition of the motor cortex, such as focal dystonia and epilepsy. While the behavioral relevance of this sexual dimorphism in human neuroplasticity remains to be determined [24], animal studies have suggested that sex hormones can morphologically and physiologically regulate the hippocampus, a brain area involved in learning and memory formation, and thus modulate cognitive processes [25].

Apart from this, the results of this study propose a potential source of variability in cortical plastic changes, which should be addressed in future studies about the manipulation of human cortical neuroplasticity.

\section{References}

1. Juraska JM. Neural plasticity and the development of sex differences. Annu Rev Sex Res 1998; 9:20-38.

2. Shors TJ, Chua C, Falduto J. Sex differences and opposite effects of stress on dendritic spine density in the male versus female hippocampus. J Neurosci 2001; 21:6292-6297.

3. Shors TJ, Falduto J, Leuner B. The opposite effects of stress on dendritic spines in male vs. female rats are NMDA receptor-dependent. Eur J Neurosci 2004; 19:145-150.

4. Jonasson Z. Meta-analysis of sex differences in rodent models of learning and memory: a review of behavioral and biological data. Neurosci Biobehav Rev 2005; 28:811-825.

5. Foy MR. 17Beta-estradiol: effect on CA1 hippocampal synaptic plasticity. Neurobiol Learn Mem 2001; 76:239-252.

6. McEwen B. Estrogen actions throughout the brain. Recent Prog Horm Res 2002; 57:357-384.

7. Woolley CS. Effects of oestradiol on hippocampal circuitry. Novartis Found Symp 2000; 230:173-180; discussion 181-177.

8. Kimura D. Review. Sex hormones influence human cognitive pattern Neuroendocrinol Lett 2002; 23(Suppl 1):67-77.

9. Nitsche MA, Paulus W. Excitability changes induced in the human motor cortex by weak transcranial direct current stimulation. J Physiol 2000; 527 (Pt 3):633-639.

10. Nitsche MA, Paulus W. Sustained excitability elevations induced by transcranial DC motor cortex stimulation in humans. Neurology 2001; 57:1899-1901.

11. Nitsche MA, Nitsche MS, Klein CC, Tergau F, Rothwell JC, Paulus W. Level of action of cathodal DC polarisation induced inhibition of the human motor cortex. Clin Neurophysiol 2003; 114:600-604.

12. Nitsche MA, Fricke $K$, Henschke U, et al. Pharmacological modulation of cortical excitability shifts induced by transcranial direct current stimulation in humans. J Physiol 2003; 553 (Pt 1):293-301.

13. Liebetanz D, Nitsche MA, Tergau F, Paulus W. Pharmacological approach to the mechanisms of transcranial DC-stimulation-induced after-effects of human motor cortex excitability. Brain 2002; 125 (Pt 10):2238-2247.

14. Smith MJ, Keel JC, Greenberg BD, et al. Menstrual cycle effects on cortical excitability. Neurology 1999; 53:2069-2072.

15. Smith MJ, Adams LF, Schmidt PJ, Rubinow DR, Wassermann EM. Effects of ovarian hormones on human cortical excitability. Ann Neurol 2002, 51:599-603.

16. Hausmann M, Tegenthoff M, Sanger J, Janssen F, Gunturkun O, Schwenkreis P. Transcallosal inhibition across the menstrual cycle: a TMS study. Clin Neurophysiol 2006; 117:26-32.

17. Herzog AG, Friedman MN, Freund S, Pascual-Leone A. Transcranial magnetic stimulation evidence of a potential role for progesterone in the modulation of premenstrual corticocortical inhibition in a woman with catamenial seizure exacerbation. Epilepsy Behav 2001; 2:367-369.

18. Maguire JL, Stell BM, Rafizadeh M, Mody I. Ovarian cycle-linked changes in $\mathrm{GABA}(\mathrm{A})$ receptors mediating tonic inhibition alter seizure susceptibility and anxiety. Nat Neurosci 2005; 8:797-804.

19. Inghilleri M, Conte A, Curra A, Frasca V, Lorenzano C, Berardelli A. Ovarian hormones and cortical excitability. An rTMS study in humans. Clin Neurophysiol 2004; 115:1063-1068.

20. Smith MD, Jones LS, Wilson MA. Sex differences in hippocampal slice excitability: role of testosterone. Neuroscience 2002; 109:517-530 
21. Yang DW, Pan B, Han TZ, Xie W. Sexual dimorphism in the induction of LTP: critical role of tetanizing stimulation. Life Sci 2004; 75: 119-127.

22. Sommer M, Alfaro A, Rummel M, et al. Half sine, monophasic and biphasic transcranial magnetic stimulation of the human motor cortex. Clin Neurophysiol 2006; 117:838-844.
23. Cahill L. Why sex matters for neuroscience. Nat Rev Neurosci 2006; 7: 477-484.

24. McCarthy MM, Konkle ATM. When is a sex difference not a sex difference? Frontiers Neuroendocrinol 2005; 26:85-102.

25. Korol DL. Role of estrogen in balancing contributions from multiple memory systems. Neurobiol Learn Mem 2004; 82:309-323. 


\subsection{Boosting focally-induced brain plasticity by dopamine}

Application of levodopa (L-DOPA) has recently been shown to improve motor as well as semantic learning in healthy subjects and patients with chronic stroke or Parkinson's disease (Knecht et al., 2004; Floel et al., 2005b; Floel et al., 2005a). The neurophysiological basis for these functional effects however has not been studied systematically so far. It has been speculated that dopamine focuses NMDA receptor-dependent cortical excitability enhancements and therefore shapes information processing (Jay, 2003; O'Donnell, 2003; Seamans and Yang, 2004). We applied L-DOPA or placebo medication to healthy subjects in combination with tDCS and PAS. tDCS induces long-lasting, stimulation-polarity-specific neuroplastic excitability enhancements or reductions, which globally involve the cortical area under the electrode, while PAS induces synapse-specific focal neuroplastic excitability changes. Under L-DOPA, the excitability enhancement elicited by anodal tDCS was reversed into inhibition, whereas the cathodal tDCS-induced excitability diminution was prolonged and consolidated. Conversely, L-DOPA facilitated the synapse-specific excitability increase induced by PAS. This pattern of results supports the notion that dopamine has a focusing effect on synapse-specific excitability-enhancing neuroplasticity in human cortical networks and it may explain the task-specific beneficial influence of dopamine on cognitive tasks. 


\title{
Boosting focally-induced brain plasticity by dopamine
}

Min-Fang Kuo M.D., Walter Paulus M.D., Michael A Nitsche M.D.

Dept. Clinical Neurophysiology, Georg-August-University Göttingen, Robert-Koch-Str. 40, 37075 Göttingen, Germany,

Phone: +551 396650; Fax: +551 398126

Key words: dopamine, human, motor cortex, neuroplasticity, paired associative stimulation, transcranial direct current stimulation

\begin{abstract}
Dopamine simultaneously produces both excitation and inhibition in the human cortex. In order to shed light on the functional significance of these seemingly opposing effects, we administered the dopamine precursor levodopa (L-dopa) to healthy subjects in conjunction with two neuroplasticity-inducing motor cortex stimulation protocols. Transcranial direct current stimulation (tDCS) induces cortical excitability enhancement by anodal and depression by cathodal brain polarization, which is not restricted to specific subgroups of synapses. In contrast, paired associative stimulation (PAS) induces focal excitability enhancements of somatosensory and motor cortical neuronal synaptic connections. Here we show that administering L-dopa turns the unspecific excitability enhancement caused by anodal tDCS into inhibition and prolongs the cathodal tDCS-induced excitability diminution. Conversely, it stabilises the PAS-induced synapse-specific excitability increase. Most importantly, it prolongs all of these after-effects by a factor of about 20. Hereby dopamine focuses synapsespecific excitability-enhancing neuroplasticity in human cortical networks.
\end{abstract}




\section{Introduction}

The neuromodulator dopamine (DA) influences cognitive, emotional, motivational and motor processes. These are consequently affected in diseases with disturbed dopamine function such as Parkinson's syndrome or schizophrenia (Grace AA et al., 1998). DAergic modulation on cognitive functions has long been investigated intensively, and the results at first glance revealed that DA enhancing agents facilitated working and long-term memory while DA receptor blockers impaired it (Bartholomeusz CF et al., 2003; Floel A et al., 2005; Kimberg DY et al., 1997; Knecht S et al., 2004; Luciana M and PF Collins, 1997; Luciana M et al., 1992; Mehta MA et al., 1999; Mehta MA et al., 2001; Muller U et al., 1998). However, the DAergic influence on cognition might be not quite as simple as originally thought: It might depend on dosage, dopaminergic sub-receptor specifity, and task characteristics (Floresco SB and AG Phillips, 2001; Kulisevsky J, 2000). It is proposed that DA improves cognitive functions by focusing information processing (Foote SL and JH Morrison, 1987), i.e. enhancing the signal to noise ratio. Specifically, DA might suppress moderate neuronal background activity, but enhance task-related high-level activity (Seamans JK and CR Yang, 2004). Such a focusing mechanism is particularly necessary for consolidation of cognitive functions related to learning and memory, which require long-term stabilization of one or a set of limited representations and suppression of other non-significant inputs in neural networks. Several studies have suggested the importance of DAergic focusing on cognition in humans (Bartholomeusz CF et al., 2003; Floel A et al., 2005; Kimberg DY et al., 1997; Knecht S et al., 2004; Mehta MA et al., 1999; Muller U et al., 1998), but direct neurophysiological evidence for such a focusing action of DA in humans is still missing. 
Here we compare the impact of DA on focal cortical neuroplasticity versus relatively non-focal, global cortical neuroplasticity. Focal plasticity was induced by paired associative stimulation (PAS). Here repetitive peripheral nerve stimulation is paired with transcranial magnetic stimulation (TMS) of the human motor cortex (Stefan K et al., 2000). It is postulated that PAS-induced excitability changes specifically facilitate somatosensory-motor cortical connections. Recently, it has moreover been demonstrated that the effects of PAS in the human motor cortex are restricted to the motor cortical representations affected by the stimulation protocol, but do not spread to neighboured ones (Weise D et al., 2006). Furthermore, the efficacy of PAS to induce motor cortical excitability alterations specifically depends on the interstimulus interval (Wolters A et al., 2003). Thus, PAS shares some critical features of associative synaptic LTP and LTD (Stefan K et al., 2002; Stefan K et al., 2000).

For induction of a broader, less spatially restricted neuroplasticity, we applied transcranial direct current stimulation (tDCS). tDCS leads to a modulation of cortical network plasticity by application of weak direct currents through the surface of the scalp. Depending on stimulation duration anodal tDCS enhances and cathodal tDCS diminishes cortical excitability for about an hour after the end of stimulation (Nitsche MA et al., 2003; Nitsche MA and W Paulus, 2001). The primary mechanism is a modulation of resting membrane potential, and the resulting polarity-specific excitability and changes in cortical activity subsequently induce changes in synaptic strength - which are however not restricted to specific synaptic subgroups, since excitability and activity of a broad range of cortical neurons is modulated by tDCS, as shown in animal experiments (Purpura DP and JG McMurtry, 1965).

Both plasticity-inducing stimulation protocols induce long-lasting, NMDA receptordependent neuroplastic excitability changes (Nitsche MA et al., 2003; Stefan K et al., 2002). The main difference lies in the specific focal effects of PAS on a restricted 
subgroup of synapses, as opposed to the plasticity induced by tDCS, which is synaptically driven but not restricted to specific subgroups of synapses. PAS-induced plasticity is also associative and timing-dependent, compared to the tonic neuronal polarization by tDCS.

We hypothesized that the impact of L-dopa on both kinds of neuroplasticity might differ. According to a proposed focusing effect of dopamine, L-dopa might enhance focal, excitability-enhancing, PAS-induced plasticity, while it might diminish increases in global cortical network excitability as generated by anodal tDCS. Conversely, global network excitability diminutions, as induced by cathodal tDCS, might be strengthened by L-dopa, and thus further increase the signal to noise ratio (Seamans JK and CR Yang, 2004). This proposed pattern of results would offer a neurophysiological explanation for the beneficial effect of DA on cognition and furthermore help to understand the pathophysiology of neuropsychiatric diseases accompanied by DA malfunction. Alternatively, it might be argued that differences in the effect of L-dopa on tDCS- and PAS-generated neuroplasticity are due to the fact that PAS mimics physiologically-occurring events more closely than the gross transcranial DC stimulation.

\section{Materials and Methods}

\section{Subjects}

Seven (three men, four women; aged $26 \pm 4$ years, PAS) and 11 (five men, six women, aged $24 \pm 4$ years, tDCS) neurologically healthy subjects participated in both experiments. The study was approved by the ethics committee of the University of Goettingen, and we conform to the Declaration of Helsinki. All subjects gave their written informed consent. 


\section{Transcranial direct current stimulation (tDCS)}

tDCS was carried out with a pair of saline-soaked surface sponge electrodes $\left(35 \mathrm{~cm}^{2}\right)$ with one of the electrodes placed over the representational area of the right abductor digiti minimi muscle (ADM) as determined by TMS and the other electrode above the right orbit as reference. The currents ran continuously for 13 (anodal tDCS) or 9 (cathodal tDCS) minutes with an intensity of $1 \mathrm{~mA}$. In previous studies, these stimulation durations have been shown to induce after-effects of tDCS lasting for about one hour (Nitsche MA et al., 2003; Nitsche MA and W Paulus, 2001).

\section{Paired associative stimulation (PAS)}

Peripheral nerve stimulation was applied on the right ulnar nerve at the level of the wrist. Single-pulse TMS was delivered over the representing area of the right ADM and preceded by an ulnar nerve stimulus with an interval of $25 \mathrm{~ms}$ with stimulation intensity of $300 \%$ of the perceptual threshold. Ninety pairs were applied at $0.05 \mathrm{~Hz}$ over 30 min, which has been shown to induce a long-lasting excitability enhancement in the motor cortex (Stefan K et al., 2000).

\section{Pharmacological Interventions}

$100 \mathrm{mg}$ L-dopa (combined with $20 \mathrm{mg}$ domperidon) or equivalent placebo (PLC) drugs were taken by the subjects one hour before the start of the experimental session. By this means, the verum drug induces a stable plasma level and produces prominent effects in the central nervous system (Floel A et al., 2005). Each experimental session was carried out in a randomized order and was separated by at least one week to avoid cumulative drug effects.

\section{Experimental procedures}

The experiments were conducted in a repeated measurement design. Subjects were seated comfortably in a reclining chair. First the optimal position of the magnetic coil 
for eliciting MEPs in the resting ADM was assessed over the left motor cortex and 20 MEPs were recorded for the first baseline. Sixty minutes after intake of the medication, a second baseline was determined to control for a possible influence of the drug on cortical excitability and adjusted if necessary.

In both tDCS and PAS experiments, 20 MEPs were recorded every five minutes for half an hour, and then every 30 minutes until two hours after the end of each intervention. For the L-dopa conditions, TMS recordings were performed at four additional time points: same day evening (se), next morning (nm), next noon (nn), and next evening (ne).

\section{Data analysis and statistics}

MEP amplitude means were calculated for each time bin, including both baseline values. The post-intervention MEPs were normalized and are given as ratios of the baseline determined immediately before intervention.

Repeated measurement ANOVAs for the time bins up to 120 min after tDCS (experiment 1) or PAS (experiment 2) were calculated with the independent variables time course, current stimulation (anodal and cathodal tDCS, for experiment 1), drug condition, and the dependent variable MEP amplitude. Student's t-tests (paired samples, two-tailed, $P<0.05)$ were performed to determine whether the MEP amplitudes before and after the interventional brain stimulations differed in each intervention condition, and if those differences depended on the drug conditions. Additional post-hoc-tests were performed to explore if L-dopa modified baseline MEPs.

\section{Results}


Baseline MEP amplitudes before intervention did not differ significantly before or after drug intake in all conditions ( $P</=0.38$, Student's $t$-tests, paired, two-tailed).

Effects of L-dopa on tDCS-induced excitability shifts of motor cortex (experiment 1)

The ANOVA revealed significant main effects of the drug, tDCS and time course, and significant interactions of tDCS $\times$ drug, drug $\times$ time course, tDCS $\times$ time course, and tDCS $\times$ drug $\times$ time course (Table 1 ). In the PLC conditions, the anodal tDCSinduced excitability increase remained significant until 30 min after stimulation, and the cathodal tDCS-induced inhibition lasted until 90 min after stimulation. As revealed by post hoc $t$-tests (paired, two-tailed, $P<0.05$ ), anodal tDCS under L-dopa resulted in a significant excitability reduction compared to baseline MEPs and the PLC condition, and the effect continued to be significant until the evening one day after tDCS (Fig. 1). Cathodal tDCS decreased motor cortex excitability under both PLC and L-dopa medication. However while this excitability decrease lasted until the morning after tDCS under L-dopa, it had already returned to baseline values $120 \mathrm{~min}$ after tDCS under PLC medication.

Effects of L-dopa on PAS-induced excitability shifts of motor cortex (experiment 2)

As shown by the ANOVA, the effects of PAS and time course are significant. The excitatory shift of MEP amplitudes returned to baseline 20 min after PAS in the PLC condition, as revealed by Student's $t$-tests (paired, two-tailed, $P<0.05$ ), while L-dopa enhanced and prolonged the excitatory effects of PAS until the evening. The differences in MEP amplitude changes between L-dopa and PLC medication conditions were significant at time points of 5, 20, 25, 90, and 120 min after PAS (Fig. 2).

\section{Discussion}


The results of the current experiments, which test the impact L-dopa on different kinds of neuroplasticity induced in the human motor cortex, are important in three aspects: 1) DA facilitates the efficacy of focal excitatory inputs to cortical networks, as provided by PAS, 2) It concomitantly inverses global cortical network excitatory plasticity modulation and stabilizes global excitability depression. As such, any tDCSinduced excitation was abolished under L-dopa; in particular anodal tDCS no longer increased but instead reduced motor cortex excitability. 3) Both PAS and tDCSinduced neuroplastic after-effects were dramatically prolonged by L-dopa by a factor of about 20.

The techniques used here thus allow for the first time to separate two different dopaminergic mechanisms in the human cortex and to determine its time course. The results are in accordance with the DA focusing hypothesis. However, it cannot be ruled out that the PAS protocol mimics physiologically-occurring events more closely than the gross tDSC stimulation and that this difference between the stimulation protocols has contributed to the results.

Similar to foregoing studies, PAS, which is supposed to induce neuroplasticity specifically in specific motor cortical synapses, resulted in a motor cortex excitability enhancement lasting for about $15 \mathrm{~min}$ after the end of stimulation in the PLC medication condition. L-dopa increased and consolidated this focal motor cortex excitability enhancement until the evening of the stimulation day. This enhancing and stabilizing effect of DA on focal excitability-enhancing neuroplasticity is consistent with the findings obtained in animal studies, in which DA facilitates associative LTP in vivo (Gurden $\mathrm{H}$ et al., 2000; Jay TM et al., 1996). These results also extend the findings of other groups, who recently showed that PAS-induced plasticity was absent in patients with Parkinson's disease when off medication, but that it was restored by L-dopa (Ueki Y et al., 2006). Here it is shown that DA is not only necessary to induce 
this kind of neuroplasticity, but that it also strengthens and consolidates it. D1 receptors might be candidates for these effects, because they are critically involved in the induction and stabilisation of LTP in animal experiments and important for learning and memory formation in humans (Floel A et al., 2005; Muller U et al., 1998).

Global cortical plasticity alterations are distinctly suppressed by DA by reversing excitation into inhibition and by prolongation of inhibitory after-effects. The prolonged inhibition elicited by cathodal tDCS under L-dopa is in line with the result from a previous tDCS study in which the predominant D2 agonist pergolide was administered (Nitsche MA et al., 2006) and this might be due to a D2 receptordependent stabilizing effect on inhibitory neuroplasticity (Otani S et al., 1998; Seamans JK and CR Yang, 2004). The reversal of the anodal tDCS-generated neuroplastic excitability enhancement into inhibition is, although in accordance with the focusing hypothesis, more difficult to explain. It could be D2 receptor-dependent due to the activity-reducing effect of this receptor. On the other hand, a D1-mediated inhibition could not be ruled out (Williams GV and SA Castner, 2006). Further studies are required to offer evidence for the speculated mechanism mentioned above. The third important aspect of this study is that these effects of DA are not restricted to the neuroplasticity induction phase, but work over a much longer time course. This longer time course might be important to consolidate focal neuroplastic excitability enhancements and global excitability diminutions. The dual consolidating effect could improve learning as well as memory formation and stabilization, because it might guarantee a prolonged activity of learning-related neuronal connections, while potentially distractive activity is minimized. Through this specific effect, DA is a candidate to work as a cognitive enhancer. 
Taken together, the most compelling result of this study is that, depending on the focality, and maybe similarity to physiologically induced plasticity of neuroplastic excitability enhancements, DA exerts a prolonged inhibitory or facilitatory effect on neuroplasticity in the human motor cortex. This effect of DA might be one important neurophysiological foundation for its beneficial behavioural effects. It has been demonstrated that learning involves specific enhancements of synaptic strength induced by activity-dependent coincident firing of pre- and postsynaptic neurons involved in the learning process (Buonomano DV and MM Merzenich, 1998; RioultPedotti MS et al., 2000). On the contrary, uncontrolled network excitability enhancements not restricted to learning-related synapses would facilitate not only task-specific synaptic connections, but also alternative ones, thus compromising the selective stabilisation of learning-related neuronal connections and consequently impairing memory formation. A focal excitability increase of neuronal networks, enhancing the excitability and synaptic strength of learning-related neuronal connections, but inhibiting the excitability of others, would therefore be a promising way to improve learning and memory formation. Exactly this seems to be what DA is doing, as shown so far primarily in animal experiments (Sawaguchi T et al., 1990) and computational models (Dreher JC et al., 2002; Durstewitz D et al., 1999) - and now also in humans. Thus this view of the neuroplasticity-modifying effect of DA might explain how it improves learning and memory formation.

\section{Acknowledgement}

We thank C. Crozier for help with the manuscript. M-F Kuo is supported by DFG (German Research Society) -funded European Graduiertenkolleg 632, “Neuroplasticity: from molecules to systems”. 


\section{References}

Bartholomeusz CF, Box G, Van Rooy C, Nathan PJ. 2003. The modulatory effects of dopamine D-1 and D-2 receptor function on object working memory in humans. J Psychopharmacol 17: 9-15.

Buonomano DV, Merzenich MM. 1998. Cortical plasticity: From synapses to maps. Annu Rev Neurosci 21: 149-186.

Dreher JC, Guigon E, Burnod Y. 2002. A model of prefrontal cortex dopaminergic modulation during the delayed alternation task. J Cogn Neurosci 14: 853-865.

Durstewitz D, Kelc M, Gunturkun O. 1999. A neurocomputational theory of the dopaminergic modulation of working memory functions. J Neurosci 19: 28072822.

Floel A, Breitenstein C, Hummel F, Celnik P, Gingert C, Sawaki L, Knecht S, Cohen LG. 2005. Dopaminergic influences on formation of a motor memory. Ann Neurol 58: 121-130.

Floresco SB, Phillips AG. 2001. Delay-dependent modulation of memory retrieval by infusion of a dopamine D1 agonist into the rat medial prefrontal cortex. Behav Neurosci 115: 934-939.

Foote SL, Morrison JH. 1987. Extrathalamic modulation of cortical function. Annu Rev Neurosci 10: 67-95.

Grace AA, Gerfen CR, Aston-Jones G. 1998. Catecholamines in the central nervous system. Overview. Adv Pharmacol 42: 655-670.

Gurden H, Takita M, Jay TM. 2000. Essential role of D1 but not D2 receptors in the NMDA receptor-dependent long-term potentiation at hippocampal-prefrontal cortex synapses in vivo. J Neurosci 20: art. no.-RC106. 
Jay TM, Burette F, Laroche S. 1996. Plasticity of the hippocampal-prefrontal cortex synapses. J Physiol Paris 90: 361-366.

Kimberg DY, Desposito M, Farah MJ. 1997. Effects of bromocriptine on human subjects depend on working memory capacity. Neuroreport 8: 3581-3585.

Knecht S, Breitenstein C, Bushuven S, Wailke S, Kamping S, Floel A, Zwitserlood P, Ringelstein EB. 2004. Levodopa: faster and better word learning in normal humans. Ann Neurol 56: 20-26.

Kulisevsky J. 2000. Role of dopamine in learning and memory: implications for the treatment of cognitive dysfunction in patients with Parkinson's disease. Drugs Aging 16: 365-379.

Luciana M, Collins PF. 1997. Dopaminergic modulation of working memory for spatial but not object cues in normal humans. J Cogn Neurosci 9: 330-347.

Luciana M, Depue RA, Arbisi P, Leon A. 1992. Facilitation Of Working Memory In Humans By A D2-Dopamine Receptor Agonist. J Cogn Neurosci 4: 58-68.

Mehta MA, Sahakian BJ, McKenna PJ, Robbins TW. 1999. Systemic sulpiride in young adult volunteers simulates the profile of cognitive deficits in Parkinson's disease. Psychopharmacology 146: 162-174.

Mehta MA, Swainson R, Ogilvie AD, Sahakian BJ, Robbins TW. 2001. Improved short-term spatial memory but impaired following the dopamine D-2 agonist bromocriptine reversal learning in human volunteers. Psychopharmacology 159: $10-20$.

Muller U, von Cramon DY, Pollmann S. 1998. D1-versus D2-receptor modulation of visuospatial working memory in humans. J Neurosci 18: 2720-2728.

Nitsche MA, Fricke K, Henschke U, Schlitterlau A, Liebetanz D, Lang N, Henning S, Tergau F, Paulus W. 2003. Pharmacological modulation of cortical excitability 
shifts induced by transcranial direct current stimulation in humans. J Physiol 553: 293-301.

Nitsche MA, Lampe C, Antal A, Liebetanz D, Lang N, Tergau F, Paulus W. 2006. Dopaminergic modulation of long-lasting direct current-induced cortical excitability changes in the human motor cortex. Eur J Neurosci 23: 1651-1657.

Nitsche MA, Nitsche MS, Klein CC, Tergau F, Rothwell JC, Paulus W. 2003. Level of action of cathodal DC polarisation induced inhibition of the human motor cortex. Clin Neurophysiol 114: 600-604.

Nitsche MA, Paulus W. 2001. Sustained excitability elevations induced by transcranial DC motor cortex stimulation in humans. Neurology 57: 18991901.

Otani S, Blond O, Desce JM, Crepel F. 1998. Dopamine facilitates long-term depression of glutamatergic transmission in rat prefrontal cortex. Neuroscience 85: 669-676.

Purpura DP, McMurtry JG. 1965. Intracellular activities and evoked potential changes during polarization of motor cortex. J Neurophysiol 28: 166-185.

Rioult-Pedotti MS, Friedman D, Donoghue JP. 2000. Learning-induced LTP in neocortex. Science 290: 533-536.

Sawaguchi T, Matsumura M, Kubota K. 1990. Catecholaminergic effects on neuronal activity related to a delayed response task in monkey prefrontal cortex. J Neurophysiol 63: 1385-1400.

Seamans JK, Yang CR. 2004. The principal features and mechanisms of dopamine modulation in the prefrontal cortex. Prog Neurobiol 74: 1-58.

Stefan K, Kunesch E, Benecke R, Cohen LG, Classen J. 2002. Mechanisms of enhancement of human motor cortex excitability induced by interventional paired associative stimulation. J Physiol 543: 699-708. 
Stefan K, Kunesch E, Cohen LG, Benecke R, Classen J. 2000. Induction of plasticity in the human motor cortex by paired associative stimulation. Brain $123 \mathrm{Pt} 3$ : $572-584$.

Ueki Y, Mima T, Ali Kotb M, Sawada H, Saiki H, Ikeda A, Begum T, Reza F, Nagamine T, Fukuyama H. 2006. Altered plasticity of the human motor cortex in Parkinson's disease. Ann Neurol 59: 60-71.

Weise D, Schramm A, Stefan K, Wolters A, Reiners K, Naumann M, Classen J. 2006. The two sides of associative plasticity in writer's cramp. Brain 129: 2709-2721. Williams GV, Castner SA. 2006. Under the curve: critical issues for elucidating D1 receptor function in working memory. Neuroscience 139: 263-276.

Wolters A, Sandbrink F, Schlottmann A, Kunesch E, Stefan K, Cohen LG, Benecke R, Classen J. 2003. A temporally asymmetric Hebbian rule governing plasticity in the human motor cortex. J Neurophysiol 89: 2339-2345. 


\section{Table 1. Results of the ANOVAs}

In both experiments, the ANOVAs encompass the time course up to $120 \mathrm{~min}$ after tDCS or PAS, because the remaining time-points were only measured for the L-dopa conditions.

\begin{tabular}{|l|l|l|l|l|}
\hline & Parameters & d.f. & F-value & $P$-value \\
\hline Experiment 1 & tDCS & 1 & 40.953 & $<0.001$ \\
& Drug & 1 & 18.059 & 0.002 \\
& Time course & 10 & 2.682 & 0.006 \\
& tDCS x Drug & 1 & 36.017 & $<0.001$ \\
& tDCS x Time course & 10 & 5.914 & $<0.001$ \\
& Drug x Time Course & 10 & 2.129 & 0.029 \\
& tDCS x Drug x Time course & 10 & 3.910 & $<0.001$ \\
\hline Experiment 2 & Drug & 1 & 11.537 & 0.015 \\
& Time course & 10 & 6.022 & $<0.001$ \\
& Drug x Time Course & 10 & 1.503 & 0.161 \\
\hline
\end{tabular}




\section{Figures}

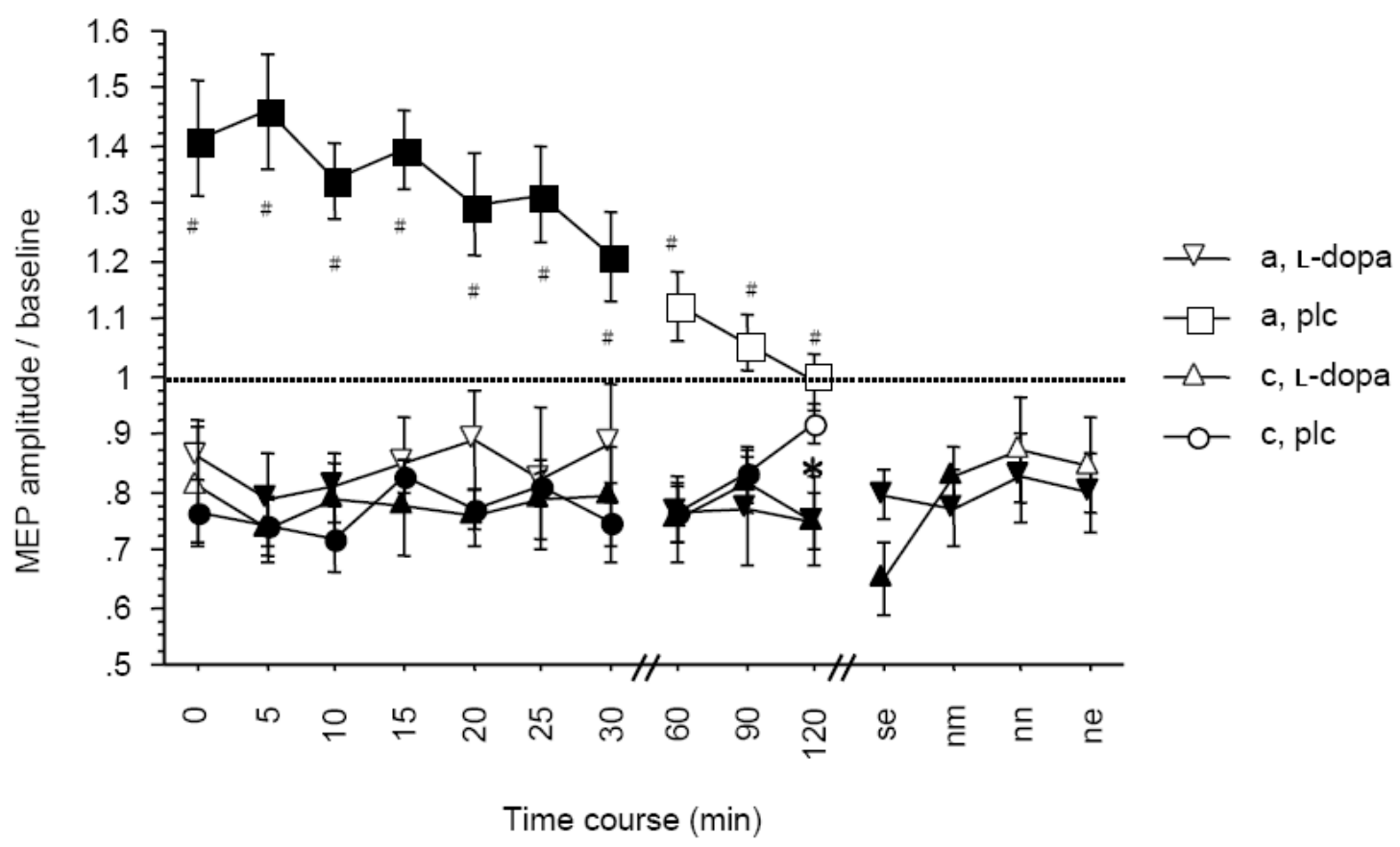

Figure 1. DAergic modulation of tDCS-induced global neuroplasticity

L-dopa reverses the anodal tDCS-elicited neuroplastic excitability enhancement, as recorded by TMS-elicited MEP amplitudes into inhibition. This inhibitory effect lasted until the evening after stimulation. A prolonged inhibition was also observed in the cathodal tDCS condition under L-dopa. Filled symbols indicate significant deviations from baseline with regard to each drug condition. Hash symbols indicate significant differences in anodal tDCS-induced excitability changes between PLC and L-dopa conditions, asterisks represent significant differences in inhibition caused by cathodal stimulation between the PLC and L-dopa medication conditions. (Student's $t$-test, two-tailed, repeated measures, $P<0.05)$. a: anodal; c: cathodal. Error bars indicate S.E.M. 


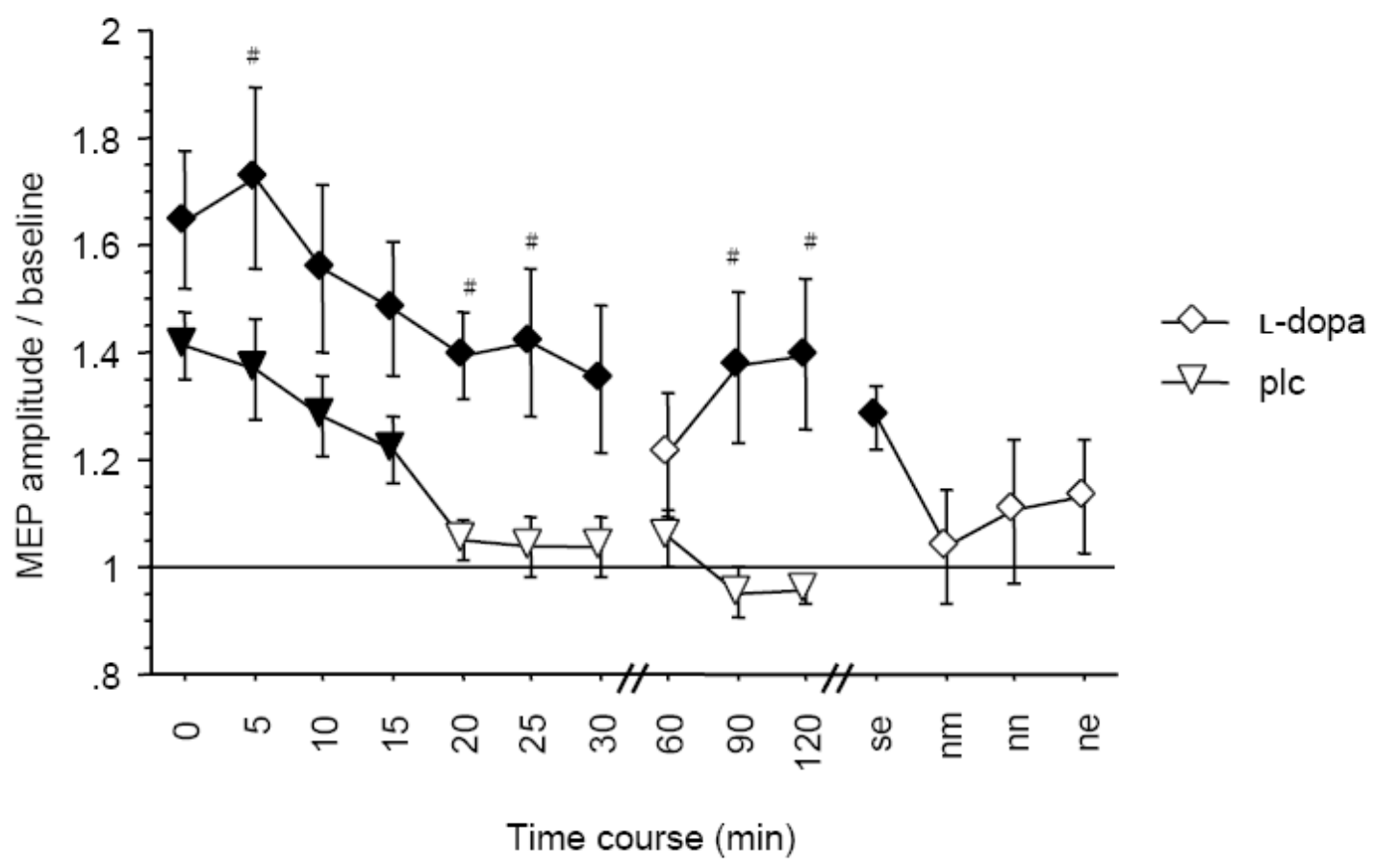

Figure 2. DA enhances and consolidates focal PAS-induced neuroplasticity

The PAS-induced excitability enhancement was prolonged under DA until the evening of PAS, while MEP amplitudes returned to baseline 15 minutes after PAS in the PLC medication condition. Hash symbols represent significant differences between PLC and L-dopa medication conditions; filled symbols indicate significant deviations from baseline with regard to each drug condition. (Student's $t$-test, twotailed, repeated measures, $P<0.05$ ). a: anodal; c: cathodal. Error bars indicate S.E.M. 


\subsection{Focusing effect of acetylcholine on neuroplasticity in the human motor cortex}

The physiological effects of ACh serve to enhance the influence of feed-forward afferent input to the cortex while decreasing background activity by suppressing excitatory feedback connections within cortical circuits (Hasselmo and Bower, 1992; Hasselmo and Barkai, 1995; Sarter et al., 2005). However, the cholinergic facilitation of LTP could not be universally replicated on the behavioral level, which implies a dual effect of ACh on the regulation of cognitive functions (Hasselmo, 2006). We explored the effects of ACh on global and synapse-specific cortical plasticity induced by tDCS and PAS respectively in order to elucidate the neurophysiological mechanism for the cholinergic facilitation of cognitive functions. Co-administration of the acetylcholinesterase-inhibitor rivastigmine with these brain stimulation techniques was demonstrated to act in the human motor cortex by reducing background noise and enhancing input-specific cortical plasticity. The findings suggest a cholinergic focusing effect that optimizes information processing and memory encoding in human cognition. 


\section{Focusing effect of acetylcholine on neuroplasticity in the human motor cortex}

Min-Fang Kuo ${ }^{1}$ M.D., Felipe Fregni ${ }^{2}$ M.D. PhD, Walter Paulus ${ }^{1}$ M.D., Michael A Nitsche ${ }^{1}$ M.D.

${ }^{1}$ Dept. Clinical Neurophysiology, Georg-August-University Göttingen, Robert-Koch-Str. 40, 37075 Göttingen, Germany,

${ }^{2}$ Harvard Center for Noninvasive Brain Stimulation, 330 Brookline Ave, Kirstein Building 452, Boston, MA 02215

Key words: neuroplasticity, acetylcholine, transcranial direct current stimulation, paired associative stimulation, motor cortex, human

\section{Abstract}

Cholinergic neuromodulation is pivotal for arousal, attention, and cognitive processes. Loss or dysregulation of cholinergic inputs leads to cognitive impairments like those manifested in Alzheimer's disease. Such dysfunction can be at least partially restored by an increase of acetylcholine (ACh). In animal studies, ACh selectively facilitates long-term excitability changes induced by feed-forward afferent input. Consequently, it has long been thought possible that ACh enhances the signal-to-noise ratio of input processing. However, the neurophysiological foundation for its ability to enhance cognition in humans is not well documented. In this study we explore the effects of rivastigmine, a cholinesterase inhibitor, on global and synapse-specific forms of cortical plasticity induced by transcranial direct current stimulation (tDCS) and paired associative stimulation (PAS) on seven to 12 healthy subjects, respectively. Rivastigmine essentially blocked the induction of the global excitability enhancement elicited by anodal tDCS, but stabilized cathodal tDCS-induced inhibitory after-effects. 
On the other hand, the synapse-specific excitability enhancement produced by PAS was facilitated by rivastigmine. These findings suggest that there is a cholinergic focusing effect that optimizes the detection of relevant signals during information processing in humans.

\section{Introduction}

Extensive evidence concerning cholinergic modulation of several cognitive functions supports an important role of acetylcholine (ACh) in arousal, attention, learning and memory formation (Gold, 2003; Sarter et al., 2003). In Alzheimer's disease, enhancing cerebral ACh level has been shown to improve impaired learning and memory functions caused by cholinergic dysfunction. With regard to its specific functional properties, neurophysiological data from animal studies reveal dual neuromodulatory effects of ACh on cortical excitability and synaptic plasticity (Rasmusson, 2000; Gu, 2002). Cholinergic blockade has been shown to reduce longterm potentiation (LTP), while cholinergic agonists enhance LTP in the hippocampus, piriform cortex, and neocortex (Blitzer et al., 1990; Hasselmo and Barkai, 1995). In humans, use-dependent plasticity of the human motor cortex is facilitated by an acetylcholinesterase inhibitor (AChEI) and blocked by a cholinergic antagonist (Sawaki et al., 2002; Meintzschel and Ziemann, 2005). However, it is also reported that ACh suppresses excitatory glutamatergic synaptic transmission via presynaptic inhibition at intrinsic, recurrent synapses but not afferent fibre synapses (Hasselmo and Bower, 1992; Hasselmo et al., 1995; Vogt and Regehr, 2001). This suggests a differential, activity-dependent cholinergic modification of neural networks in which ACh facilitates the detection of incoming afferent inputs while it decreases intrinsic feedback excitability, thereby focusing the encoding of relevant, associated 
information processing. Moreover, local application of scopolamine, a muscarinic receptor antagonist, selectively impairs cognitive performance during encoding (Blokland et al., 1992; Winters and Bussey, 2005) but not retrieval (Rogers and Kesner, 2003), indicating a phase-specific action of ACh.

To test the focusing action of ACh on neuroplasticity in humans, two protocols of brain stimulation were introduced in the present study. In the paired associative stimulation (PAS) protocol, repetitive peripheral nerve stimulation is paired with transcranial magnetic stimulation (TMS) of the human motor cortex (Stefan et al., 2000). It is postulated that PAS-induced excitability changes share the features of associative synaptic LTP and LTD, depending on the sequence of the nearsynchronous pair of stimuli from different stimulation modalities in the motor cortex (Stefan et al., 2000; Wolters et al., 2003), which parallels the spike-timing-dependent rule for Hebbian LTP and LTD induction in animal studies (Dan and Poo, 2004). PAS should thus specifically induce neuroplasticity in somatosensory-motor cortical synapses. Transcranial direct current stimulation (tDCS) encompasses the global modulation of cortical network plasticity by application of weak direct currents through the surface of the scalp. Anodal tDCS enhances cortical excitability, while cathodal tDCS diminishes it for up to an hour after the end of stimulation (Nitsche and Paulus, 2000, 2001; Nitsche et al., 2003a). The primary mechanism is a modulation of resting membrane potential, and the resulting polarity-specific excitability changes subsequently induce changes of synaptic strength, which are however not restricted to specific synaptic connections (Purpura and McMurtry, 1965). Both plasticity-inducing protocols accomplish long-lasting, NMDA receptordependent excitability changes (Liebetanz et al., 2002; Stefan et al., 2002; Nitsche et al., 2003b). The main difference lies in the synapse-specific focal effects of PAS: while the plasticity induced by tDCS is relatively non-focal and not synapse-specific 
since it is thought to change cortical excitability under the whole area covered by the relatively large stimulation electrode, the plasticity induced by PAS is restricted to the inter-coritcal connections between somatosensory and motor cortex. According to the focusing hypothesis of ACh, it should selectively enhance specific synaptic modifications induced by PAS, while depressing global ones accomplished by tDCS, in order to enhance the signal-to-noise ratio in human cortical networks.

\section{Procedures and Methods}

\section{Subjects}

Seven to 12 healthy subjects (tDCS experiment: six men, six women, aged (mean \pm SD) $24 \pm 3$ years; PAS experiment: three women, four men; aged $27 \pm 4$ years) without medication participated in each experiment. Both studies were approved by the ethics committee of the University of Goettingen, and we conform to the Declaration of Helsinki. All subjects had given written informed consent.

\section{Transcranial direct current stimulation}

tDCS was carried out with a pair of saline-soaked surface sponge electrodes $\left(35 \mathrm{~cm}^{2}\right)$ with one of the electrodes placed over the representational area of the right abductor digiti minimi muscle (ADM) as determined by TMS and the other electrode above the right orbit as reference. The currents ran continuously for 13 (anodal tDCS) or 9 (cathodal tDCS) minutes with an intensity of $1 \mathrm{~mA}$. In previous studies, these stimulation durations have been shown to induce after-effects of tDCS lasting for about one hour (Nitsche and Paulus, 2001; Nitsche et al., 2003a).

\section{Paired associative stimulation}

Peripheral nerve stimulation was applied on the right ulnar nerve at the level of the wrist with a Digitimer D185 stimulator (Digitimer Ltd., Welwyn Garden City, UK). 
Single-pulse TMS was delivered over the representing area of the right ADM. Each TMS pulse, at an intensity eliciting a muscle evoked potential of about $1 \mathrm{mV}$ peak-topeak amplitude, was preceded by an ulnar nerve stimulus with an interval of $25 \mathrm{~ms}$ using a standard stimulation block (cathodal proximal) at a stimulation width of 200 $\mu$ s and stimulation intensity of $300 \%$ of the perceptual threshold, defined as the lowest intensity of the stimuli which are perceivable by the subject. Ninety pairs were applied at $0.05 \mathrm{~Hz}$ over $30 \mathrm{~min}$, which has been shown to induce a long-lasting excitability enhancement in the motor cortex (Stefan et al., 2000).

\section{Pharmacological Interventions}

Rivastigmine (3mg) or equivalent placebo (PLC) drugs were taken by the subjects two hours before the start of the intervention (Kennedy et al., 1999). This dose was chosen to minimize drug-induced side-effects, but to enhance the cholinergic level of the central nervous system effectively. The experimental sessions were carried out in a randomized order and were separated by at least one week to avoid cumulative drug or stimulation effects.

\section{Measurement of motor cortical excitability}

TMS-elicited muscle-evoked potentials (MEPs) were recorded to measure excitability changes of the representational motor cortical area of the right ADM. Single-pulse TMS was conducted by a Magstim 200 magnetic stimulator (Magstim Company, Whiteland, Dyfed, UK) with a figure-of-eight magnetic coil (diameter of one winding $=70 \mathrm{~mm}$, peak magnetic field $=2.2$ Tesla). The coil was held tangentially to the skull, with the handle pointing backwards and laterally at an angle of $45^{\circ}$ from midline. The optimal position was defined as the site where stimulation resulted consistently in the largest MEPs. Surface EMG was recorded from the right ADM with Ag-AgCl electrodes in a belly-tendon montage. The signals were amplified and filtered with a 
time constant of $10 \mathrm{~ms}$ and a low-pass filter of $2.5 \mathrm{kHz}$, then digitized at an analogueto-digital rate of $5 \mathrm{kHz}$ and further relayed into a laboratory computer using the Signal software and CED 1401 hardware (Cambridge Electronic Design, Cambridge, UK). The intensity was adjusted to elicit baseline MEPs of, on average, $1 \mathrm{mV}$ peak-to-peak amplitude and was kept constant for the post-stimulation assessment unless adjusted (see below).

\section{Experimental procedures}

The experiments were conducted in a repeated measurement design. Subjects were seated comfortably in a reclining chair. First the optimal position of the magnetic coil for eliciting MEPs in the resting ADM was assessed over the left motor cortex and 20 MEPs were recorded for the first baseline. Two hours after intake of the medication, a second baseline was determined to control for a possible influence of the drug on cortical excitability and adjusted if necessary.

In Experiment 1 with tDCS, one of the DC electrodes, to which in the following the terms cathodal or anodal tDCS refer, was fixed at the cortical representational area of $\mathrm{ADM}$ as defined during the first baseline recording, and the other one was fixed at the contralateral forehead area above the right orbit. Direct currents were applied on 12 subjects for 9 (cathodal) or 13 min (anodal). After cessation of tDCS, 20 MEPs were recorded at $0.25 \mathrm{~Hz}$ every five minutes for half an hour, and then every 30 minutes until two hours after the end of DC stimulation, since tDCS-induced after-effects without medication will not last longer than this period of time. For the rivastigmine conditions, TMS recordings were performed at four additional time points: same day evening (se), next morning (nm), next noon (nn), and next evening (ne). 
In Experiment 2 with PAS, the interventional PAS protocol as described above was employed (3) on seven subjects. TMS recording procedures were the same as described above.

\section{Data analysis and statistics}

MEP amplitude means were calculated first individually, then inter-individually for each time bin including both baseline values. The post-intervention MEPs were normalized and are given as ratios of the baseline determined immediately before intervention (tDCS/PAS).

Repeated measurement ANOVAs for the time bins up to 120 min after tDCS (experiment 1) or PAS (experiment 2) were calculated with the independent variables time course, current stimulation (anodal and cathodal tDCS, experiment 1 only), drug condition (rivastigmine vs. placebo), and the dependent variable MEP amplitude. If appropriate, post-hoc student's $t$-tests (paired samples, two-tailed, $P<0.05$ ) were performed to determine whether the MEP amplitudes before and after the interventional brain stimulations differed in each intervention condition, and if those differences depended on the drug conditions. Additional post-hoc tests (Student's ttests, $P<0.05)$ were performed to explore whether rivastigmine modified baseline MEPs.

\section{Results}

Baseline MEP amplitudes did not differ significantly before and after drug intake in all conditions. Absolute baseline MEP amplitudes were not different in all medication and stimulation subgroups (Student's $t$-tests, paired, two-tailed, $P>0.05$ ).

Effects of rivastigmine on tDCS-induced motor cortex excitability shifts (experiment 1) 
The ANOVA revealed a significant main effect of tDCS and significant interactions of tDCS $\times$ time course, and tDCS $\times$ drug $\times$ time course (Table 1$)$. In the PLC conditions, the anodal tDCS-induced excitability increase stayed significant until 30 min after tDCS, and the cathodal tDCS-induced inhibition lasted until 60 min after DC stimulation. As revealed by the post hoc t-tests (paired, two-tailed, $P<0.05$ ), rivastigmine initially abolished the induction of both the anodal tDCS-elicited excitability enhancement and the cathodal tDCS-elicited excitability diminution. However, a delayed, consolidated inhibition induced by cathodal tDCS was observed in the rivastigmine condition, as compared to PLC conditions (Fig. 1). The decrease of excitability generated by cathodal tDCS under rivastigmine remained significant for two hours after tDCS, while it returned to baseline level after $60 \mathrm{~min}$ in the PLC condition (Fig. 1).

Effects of rivastigmine on PAS-induced motor cortex excitability shifts (experiment 2) As shown by the ANOVA, the main effects of drug and time course as well as the interaction of drug and time course are significant. The excitatory shift of MEP amplitudes returned to baseline 20 min after PAS in the PLC medication condition, as revealed by Student's $t$-tests (paired, two-tailed, $P<0.05$ ), while rivastigmine enhanced and prolonged the excitatory effects of PAS. The difference of MEP amplitude changes between rivastigmine and PLC was significant for later time points (Fig. 2).

\section{Discussion}

The present study demonstrates that ACh enhances the synapse-specific cortical excitability increase induced by PAS, while it prevents global excitatory after-effects produced by anodal tDCS. ACh also delayed the induction of the cathodal tDCS- 
elicited excitability decrease and prolonged its overall duration. Since MEP amplitudes were not modified by rivastigmine in the dosage applied alone, we have no evidence for a direct cholinergic influence of the drug on cortico-spinal excitability. The results support the hypothesis of a focusing effect of ACh on neuroplasticity of cortical networks. ACh not only facilitates synapse-specific neuroplasticity but also suppresses synergistically global excitability changes. By both mechanisms ACh is well suited to improve the signal-to-noise ratio and to refine information processing in neural networks.

\section{ACh diminishes tDCS-driven neuroplasticity}

At first glance the inhibitory effect of rivastigmine on facilitatory neuroplasticity induced by anodal tDCS seems contradictory to the results obtained from animal studies, in which LTP was facilitated by cholinergic stimulation (Brocher et al., 1992; Abe et al., 1994; Hasselmo and Barkai, 1995; Patil et al., 1998). The major conceptual difference between these studies and our tDCS experiment is the kind of plasticity specific for synaptic sub-populations, induced either with paired or high-frequency suprathreshold electrical stimulation in slice preparation - as compared to the tDCSelicited neuroplasticity resulting from membrane polarization as well as global synaptic modification and thus probably not being restricted to certain subgroups of synapses. The diminution of the anodal tDCS-induced excitability enhancement might be due to an ACh-induced decrease of general excitation within global neuronal networks, probably modulated via the cholinergic presynaptic inhibition of excitatory feedback potentials or excitatory transmission at recurrent connections. Moreover, evidence reveals that the suppression of synaptic transmission is selective for recently modified synapses (Linster et al., 2003) but does not apply to silent synapses (Fernandez de Sevilla et al., 2002). Thus it is probable that synapses which are 
globally modified by tDCS in the present study are more susceptible to cholinergic suppression of synaptic transmission during plasticity induction. A similar effect is also demonstrated by a recent study using a dynamic clamp system to mimic in vivolike background activities in motor cortical slices, in which cholinergic facilitation of LTP was attenuated in the presence of random background noise (Desai and Walcott, 2006).

On the other hand, ACh consolidated inhibitory after-effects generated by cathodal tDCS, although it abolished its initial induction phase. Such a biphasic effect could be explained by a negative cholinergic regulation within different temporary profiles; as the reduction of inhibitory neuronal groups initially blocked the cathodal tDCS effect, while the later consolidation of excitability decrease could be contributed by the cholinergic inhibition of excitatory networks.

\section{ACh facilitates PAS-induced cortical excitability enhancement}

The PAS experiment demonstrates a positive cholinergic modulation of PAS-elicited synaptic-specific plasticity. It was suggested that PAS represents associative LTP in the human motor cortex (Stefan et al., 2000; Stefan et al., 2002). Thus the results of our study are in accordance with the respective animal experiments (Blitzer et al., 1990; Hasselmo and Barkai, 1995). Moreover, they are concordant with the results of recent behavioural studies in the human motor cortex exploring the effect of ACh modulation on use-dependent plasticity, which was blocked by the ACh antagonist scopolamine (Sawaki et al., 2002) and enhanced by an acetylcholinersterase inhibitor (Meintzschel and Ziemann, 2005). Therefore, our results offer a neurophysiological mechnism how ACh might improve behavoiral plasticity.

Since ACh has been shown to facilitate cortical sensory plasticity by enhancing sensory input processing (Rasmusson and Dykes, 1988; Tremblay et al., 1990; Patil et 
al., 1998) and suppressing irrelevant input (Hasselmo and Barkai, 1995), one might furthermore speculate that rivastigmine specifically improved the efficacy of PAS by enhancing the signal-to-noise ratio, thus facilitating information processing within neural networks.

\section{Summary of cholinergic modulation in human cortical plasticity}

The results of the present study suggest that ACh has fairly specific effects on cortical plasticity. It modulates external associative input and global plasticity differently, thereby leading to an increased signal-to-noise ratio. This focusing effect of ACh might also explain the positive cholinergic modulation of learning.

Its functional significance might be the more efficient information processing and memory encoding by differential cholinergic modulation. The dual effects of ACh, namely enhancing feedforward afferent input to the cortex while suppressing excitatory feedback from recently modified, recurrent connections, improve the encoding of new memory by preventing interference from synapses modified by prior learning (Atri et al., 2004). However, this hypothesis should be tested in future experiments.

Taken together, the present study is in accordance with cortical cholinergic functions enhancing the contrast of relevant stimuli against background noise or distracters and hereby improving signal processing during information encoding. Therefore it further strengthens the rational basis for the application of cholinesterase inhibitors to improve cognitive functions in patients with Alzheimer's disease or vascular dementia.

\section{Acknowledgement}

We thank Novartis for supporting this project. MF Kuo is supported by European Graduiertenkolleg 632 ,Neuroplasticity: From Molecules to Systems’ which is funded 
by German Research Society (DFG). This project was further supported by the DFGprogram “Nicotine (SPP 1226)”. 


\section{References}

Abe K, Nakata A, Mizutani A, Saito H (1994) Facilitatory but nonessential role of the muscarinic cholinergic system in the generation of long-term potentiation of population spikes in the dentate gyrus in vivo. Neuropharmacology 33:847852.

Atri A, Sherman S, Norman KA, Kirchhoff BA, Nicolas MM, Greicius MD, Cramer SC, Breiter HC, Hasselmo ME, Stern CE (2004) Blockade of central cholinergic receptors impairs new learning and increases proactive interference in a word paired-associate memory task. Behav Neurosci 118:223-236.

Blitzer RD, Gil O, Landau EM (1990) Cholinergic stimulation enhances long-term potentiation in the CA1 region of rat hippocampus. Neurosci Lett 119:207-210.

Blokland A, Honig W, Raaijmakers WG (1992) Effects of intra-hippocampal scopolamine injections in a repeated spatial acquisition task in the rat. Psychopharmacology (Berl) 109:373-376.

Brocher S, Artola A, Singer W (1992) Agonists of cholinergic and noradrenergic receptors facilitate synergistically the induction of long-term potentiation in slices of rat visual cortex. Brain Res 573:27-36.

Dan Y, Poo MM (2004) Spike timing-dependent plasticity of neural circuits. Neuron 44:23-30.

Desai NS, Walcott EC (2006) Synaptic bombardment modulates muscarinic effects in forelimb motor cortex. J Neurosci 26:2215-2226.

Fernandez de Sevilla D, Cabezas C, de Prada AN, Sanchez-Jimenez A, Buno W (2002) Selective muscarinic regulation of functional glutamatergic Schaffer collateral synapses in rat CA1 pyramidal neurons. J Physiol 545:51-63.

Gold PE (2003) Acetylcholine modulation of neural systems involved in learning and memory. Neurobiol Learn Mem 80:194-210.

Gu Q (2002) Neuromodulatory transmitter systems in the cortex and their role in cortical plasticity. Neuroscience 111:815-835.

Hasselmo ME, Bower JM (1992) Cholinergic suppression specific to intrinsic not afferent fiber synapses in rat piriform (olfactory) cortex. J Neurophysiol 67:1222-1229.

Hasselmo ME, Barkai E (1995) Cholinergic modulation of activity-dependent synaptic plasticity in the piriform cortex and associative memory function in a network biophysical simulation. J Neurosci 15:6592-6604. 
Hasselmo ME, Schnell E, Barkai E (1995) Dynamics of learning and recall at excitatory recurrent synapses and cholinergic modulation in rat hippocampal region CA3. J Neurosci 15:5249-5262.

Kennedy JS, Polinsky RJ, Johnson B, Loosen P, Enz A, Laplanche R, Schmidt D, Mancione LC, Parris WC, Ebert MH (1999) Preferential cerebrospinal fluid acetylcholinesterase inhibition by rivastigmine in humans. $\mathrm{J}$ Clin Psychopharmacol 19:513-521.

Liebetanz D, Nitsche MA, Tergau F, Paulus W (2002) Pharmacological approach to the mechanisms of transcranial DC-stimulation-induced after-effects of human motor cortex excitability. Brain 125:2238-2247.

Linster C, Maloney M, Patil M, Hasselmo ME (2003) Enhanced cholinergic suppression of previously strengthened synapses enables the formation of selforganized representations in olfactory cortex. Neurobiol Learn Mem 80:302314.

Meintzschel F, Ziemann U (2005) Modification of Practice-dependent Plasticity in Human Motor Cortex by Neuromodulators. Cereb Cortex.

Nitsche MA, Paulus W (2000) Excitability changes induced in the human motor cortex by weak transcranial direct current stimulation. J Physiol 527 Pt 3:633639.

Nitsche MA, Paulus W (2001) Sustained excitability elevations induced by transcranial DC motor cortex stimulation in humans. Neurology 57:1899-1901.

Nitsche MA, Nitsche MS, Klein CC, Tergau F, Rothwell JC, Paulus W (2003a) Level of action of cathodal DC polarisation induced inhibition of the human motor cortex. Clin Neurophysiol 114:600-604.

Nitsche MA, Fricke K, Henschke U, Schlitterlau A, Liebetanz D, Lang N, Henning S, Tergau F, Paulus W (2003b) Pharmacological modulation of cortical excitability shifts induced by transcranial direct current stimulation in humans. J Physiol 553:293-301.

Patil MM, Linster C, Lubenov E, Hasselmo ME (1998) Cholinergic agonist carbachol enables associative long-term potentiation in piriform cortex slices. J Neurophysiol 80:2467-2474.

Purpura DP, McMurtry JG (1965) Intracellular activities and evoked potential changes during polarization of motor cortex. J Neurophysiol 28:166-185.

Rasmusson DD (2000) The role of acetylcholine in cortical synaptic plasticity. Behav Brain Res 115:205-218.

Rasmusson DD, Dykes RW (1988) Long-term enhancement of evoked potentials in cat somatosensory cortex produced by co-activation of the basal forebrain and cutaneous receptors. Exp Brain Res 70:276-286. 
Rogers JL, Kesner RP (2003) Cholinergic modulation of the hippocampus during encoding and retrieval. Neurobiol Learn Mem 80:332-342.

Sarter M, Bruno JP, Givens B (2003) Attentional functions of cortical cholinergic inputs: what does it mean for learning and memory? Neurobiol Learn Mem 80:245-256.

Sawaki L, Boroojerdi B, Kaelin-Lang A, Burstein AH, Butefisch CM, Kopylev L, Davis B, Cohen LG (2002) Cholinergic influences on use-dependent plasticity. J Neurophysiol 87:166-171.

Stefan K, Kunesch E, Cohen LG, Benecke R, Classen J (2000) Induction of plasticity in the human motor cortex by paired associative stimulation. Brain $123 \mathrm{Pt}$ 3:572-584.

Stefan K, Kunesch E, Benecke R, Cohen LG, Classen J (2002) Mechanisms of enhancement of human motor cortex excitability induced by interventional paired associative stimulation. J Physiol 543:699-708.

Tremblay N, Warren RA, Dykes RW (1990) Electrophysiological studies of acetylcholine and the role of the basal forebrain in the somatosensory cortex of the cat. II. Cortical neurons excited by somatic stimuli. J Neurophysiol 64:1212-1222.

Vogt KE, Regehr WG (2001) Cholinergic modulation of excitatory synaptic transmission in the CA3 area of the hippocampus. J Neurosci 21:75-83.

Winters BD, Bussey TJ (2005) Removal of cholinergic input to perirhinal cortex disrupts object recognition but not spatial working memory in the rat. Eur $\mathrm{J}$ Neurosci 21:2263-2270.

Wolters A, Sandbrink F, Schlottmann A, Kunesch E, Stefan K, Cohen LG, Benecke R, Classen J (2003) A temporally asymmetric Hebbian rule governing plasticity in the human motor cortex. J Neurophysiol 89:2339-2345. 


\section{Table 1. Results of the ANOVAs}

In both experiments, the ANOVAs encompass the time course up to $120 \mathrm{~min}$ after tDCS or PAS, because the remaining time-points were only measured for the rivastigmine conditions. 'Drug' represents rivastigmine and placebo, while 'tDCS' indicates anodal and cathodal polarity.

\begin{tabular}{|l|l|l|l|l|}
\hline & Parameters & d.f. & F-value & $P$-value \\
\hline Experiment 1 & tDCS & 1 & 40.585 & $<0.001$ \\
& Drug & 1 & 3.650 & 0.082 \\
& Time cours & 10 & 1.700 & 0.099 \\
& tDCS x Drug & 1 & 3.417 & 0.092 \\
& tDCS x Time course & 10 & 1.868 & 0.066 \\
& Drug x Time Course & 10 & 0.290 & 0.976 \\
& tDCS x Drug x Time course & 10 & 4.206 & $<0.001$ \\
\hline Experiment 2 & Drug & 1 & 22.383 & 0.005 \\
& Time course & 10 & 7.096 & $<0.001$ \\
& Drug x Time Course & 10 & 6.062 & $<0.001$ \\
\hline
\end{tabular}




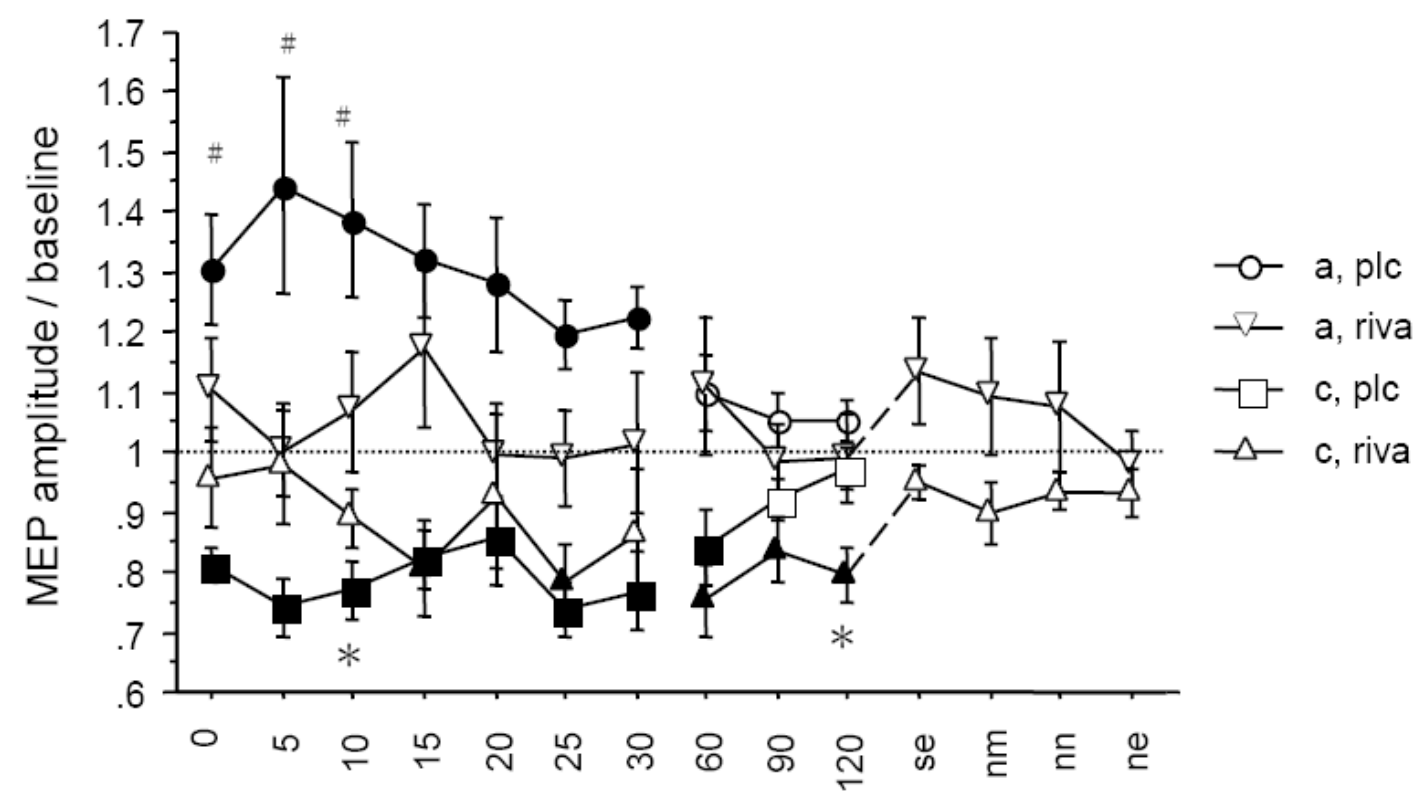

Time after tDCS $(\mathrm{min})$

\section{Figure 1. Cholinergic modulation of global cortical plasticity induced by tDCS}

Rivastigmine abolished the induction of anodal tDCS-elicited excitability increases, as recorded by TMS-evoked MEP amplitudes. Additionally, rivastigmine initially diminished the excitability reduction induced by cathodal tDCS under rivastigmine. However, the respective excitability diminution was later consolidated. Filled symbols indicate significant deviations from baseline with regard to each drug condition. Hash symbols indicate significant differences in anodal tDCS-induced excitability changes between PLC and rivastigmine conditions, asterisks represent significant differences in inhibition caused by cathodal stimulation between the PLC and rivastigmine medication conditions (Student's $t$-test, two-tailed, repeated measures, $P<0.05)$. a: anodal; c: cathodal; riva: rivastigmine. Error bars indicate S.E.M. 


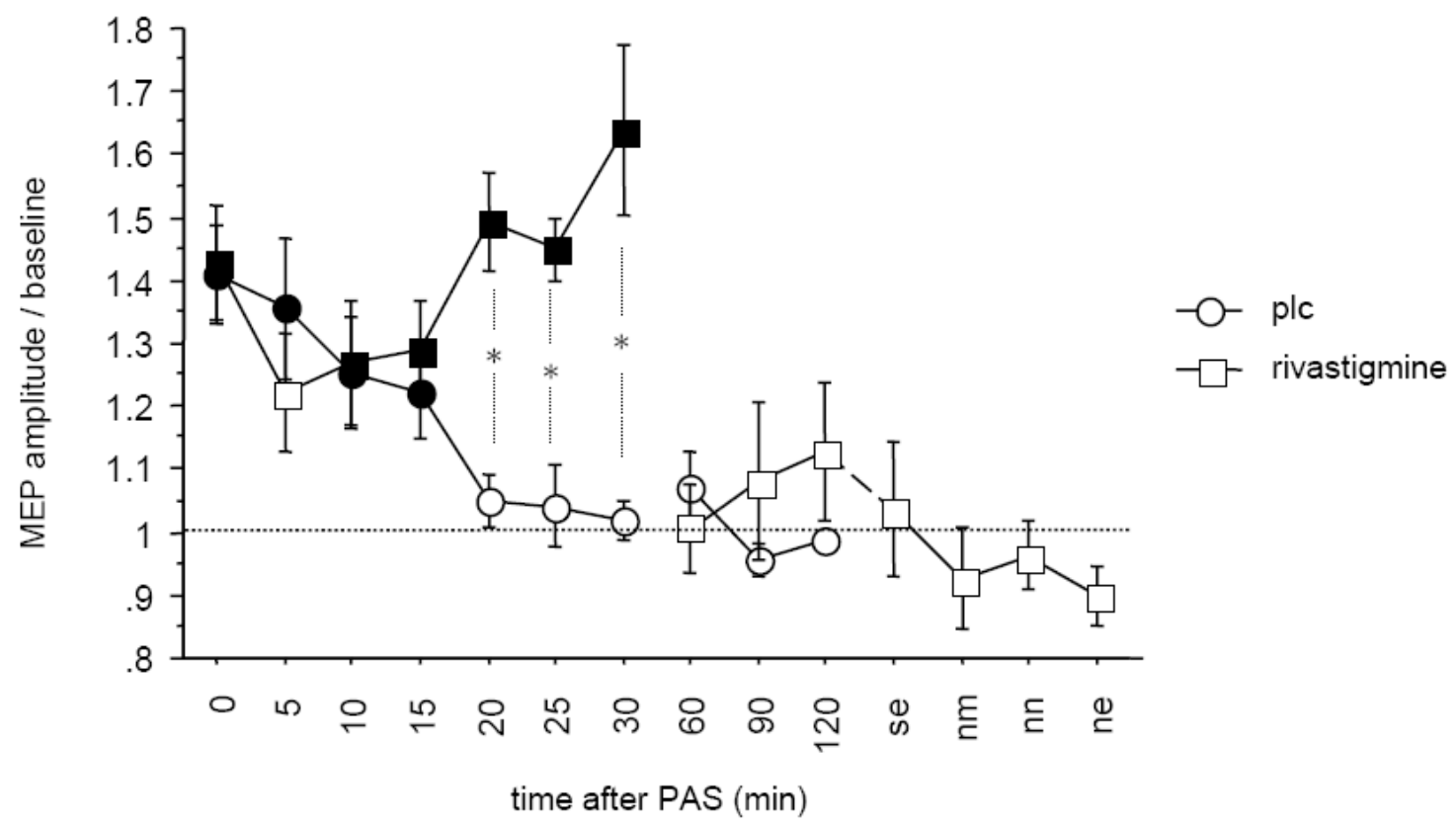

Figure 2. PAS-induced synapse-specific excitability enhancement is facilitated by ACh

The PAS-induced excitability enhancement was increased and prolonged under rivastigmine until 30 minutes post PAS, while MEP amplitudes returned to baseline 15 minutes after PAS in the PLC medication condition. Hash symbols represent significant differences between PLC and rivastigmine conditions; filled symbols indicate significant deviations from baseline with regard to each drug condition. (Student's $t$-test, two-tailed, repeated measures, $P<0.05$ ). riva: rivastigmine. Error bars indicate s.E.M. 


\subsection{Timing-dependent modulation of associative plasticity by general network excitability in the human motor cortex}

Homeostatic mechanisms have been suggested to maintain the stability of cortical neural networks, which is crucial for the modulation of neuroplasticity (Abraham and Tate, 1997; Abbott and Nelson, 2000). Specifically it is suggested that the amount and direction of associative plasticity is determined by the amount of the history of background activity. Here PAS which induces focal plastic changes serves as an analogue of learning-related modulations of cortical plasticity. By contrast, a generalized modification of the global neural excitability can be achieved by tDCS. When applied before PAS, anodal tDCS further increased the excitability-enhancing efficacy of the PAS protocol, while cathodal tDCS diminished the PAS-induced excitability enhancement and reversed it to inhibition. On the other hand, homeostatic neuroplasticity was achieved by the simultaneous application of both protocols. PAS elicited inhibitory plasticity when background excitability was increased by anodal tDCS, while the generally reduced background excitability caused by cathodal tDCS strengthened the facilitatory PAS-induced plasticity. We conclude that background network excitability influence associative plasticity. However, the relationship might be more complex than previously thought: Whereas enhanced or diminished background activity established before the induction of associative plasticity has an anti-homeostatic effect, the results accomplished by simultaneous modulation of both parameters are in accordance with homeostatic rules. These results might provide further insight into the possible neurophysiological mechanisms involved in learning and memory formation. 


\title{
Timing-Dependent Modulation of Associative Plasticity by General Network Excitability in the Human Motor Cortex
}

\author{
Michael A. Nitsche, Amelie Roth, Min-Fang Kuo, Anja K. Fischer, David Liebetanz, Nicolas Lang, Frithjof Tergau, and \\ Walter Paulus \\ Georg-August-University, Department for Clinical Neurophysiology, 37075 Goettingen, Germany
}

\begin{abstract}
Associative neuroplasticity, which encompasses the modification of synaptic strength by coactivation of two synaptic inputs, has been linked to learning processes. Because unlimited plasticity destabilizes neuronal networks, homeostatic rules were proposed and experimentally proven that control for the amount and direction of plasticity dependent on background network activity. Accordingly, low background activity would enhance facilitatory plasticity, whereas high background activity would inhibit it.

However, the impact of background excitability on associative plasticity has not been studied so far in humans. Facilitatory associative plasticity was induced by paired associative stimulation (PAS) in the human motor cortex, whereas background activity was enhanced or diminished by transcranial direct current stimulation (tDCS). When applied before PAS, excitability-enhancing tDCS also boosted the efficacy of PAS, whereas excitability-diminishing tDCS turned it into inhibition. Thus, previous background activity does not influence associative plasticity homeostatically. When tDCS and PAS were applied simultaneously, now in accordance with homeostatic rules of neuroplasticity, reduced background activity resulted in a prolonged excitability enhancement by PAS, whereas enhanced background activity turned it into inhibition. We conclude that background network activity can influence associative plasticity homeostatically. However, only simultaneous modulation of both parameters is in accordance with homeostatic concepts. These findings might be of importance for the development of plasticity-inducing stimulation protocols supporting information processing in humans.
\end{abstract}

Key words: homeostatic plasticity; paired associative stimulation; tDCS; TMS; motor cortex; human

\section{Introduction}

Understanding the neurophysiological basis of learning is an important endeavor of cognitive neuroscience research. Mechanisms, like long-term potentiation, may be involved (RioultPedotti et al., 1998, 2000; Ziemann et al., 2004; Stefan et al., 2006). Because unlimited neuroplasticity would destabilize neuronal networks, homeostatic mechanisms have been proposed to control for balanced network modifications. Homeostatic plasticity rules share the common feature that the history or actual state of global network activity influences the direction of neuroplastic changes induced by specific stimuli (Bienenstock et al., 1982; Abraham and Tate, 1997). Specifically, the effect of an actual stimulus is inversely correlated with the amount of background activity.

Homeostatic mechanisms are relevant in animal slice preparations and cultured neuronal networks (Li et al., 1998; Turrigiano et al., 1998) and at the system level in humans. In the human motor cortex, it has been shown that excitabilityenhancing fast repetitive transcranial magnetic stimulation (rTMS) increased the efficacy of a later excitability-diminishing slow rTMS protocol (Iyer et al., 2003). Moreover, enhancing or diminishing motor cortex excitability with transcranial direct

Received Dec. 12, 2006; revised March 4, 2007; accepted March 5, 2007.

This work was supported by the Bernstein Center for Computational Neuroscience (Goettingen, Germany). Correspondence should be addressed to Michael A. Nitsche at the above address. E-mail: mnitsch1@gwdg.de. D0I:10.1523/JNEUROSCI.5348-06.2007

Copyright $\odot 2007$ Society for Neuroscience $\quad$ 0270-6474/07/273807-06\$15.00/0 current stimulation (tDCS) resulted in inversely directed effects on excitability of identical rTMS protocols (Lang et al., 2004; Siebner et al., 2004). Because rTMS and tDCS induce widespread and not synapse-specific motor cortex excitability alterations, the results of these studies reflect a homeostatic regulation of background or general motor cortex excitability. However, the rules of homeostatic plasticity were originally dedicated to focal plastic changes of the strength of a limited amount of synapses relative to background activity, and these focal changes may resemble learning mechanisms more closely than global changes of excitability.

Paired associative stimulation (PAS) is an interesting tool to induce synapse-specific and thus learning-like neuroplastic modifications. It induces plasticity by simultaneous activation of intracortical neurons activated by motor cortex TMS and another population of neurons activated by somatosensory peripheral stimulation. With this selectivity, it may reflect the neurophysiological foundations of learning processes. A close relationship between motor learning and the amount of PAS-induced excitability-enhancing plasticity was demonstrated recently (Ziemann et al., 2004; Stefan et al., 2006).

We aimed to explore the impact of motor cortex background activity on associative plasticity. First (experiment 1), we identified PAS protocols resulting in short- or long-lasting excitability enhancements. We then evaluated the impact of a background activity enhancement by anodal tDCS or diminution by cathodal tDCS on subsequently (experiment 2) or simultaneously (experiment 3) applied PAS. Furthermore, we evaluated the effects of 
simultaneous tDCS and PAS for PAS protocol-eliciting longerlasting excitability enhancements (experiment 4), to study the dependency of homeostatic plasticity on the salience of plasticityinducing protocols. Because of the rules of homeostatic plasticity, excitability-diminishing cathodal tDCS should enhance, whereas excitability-enhancing anodal tDCS should reduce, the efficacy of PAS. By comparing different temporal combinations of the protocols, we aimed to explore the temporal characteristics of homeostatic plasticity. Applying salient and nonsalient PAS protocols for inducing long-lasting excitability changes, we tested the hypothesis that the quality of associative plasticity might determine whether homeostatic mechanisms apply.

\section{Materials and Methods}

Subjects. Six to 12 healthy subjects participated in each experiment. For experiments $1-3,12$ subjects were recruited (eight females; age, $25 \pm 2.5$ $\mathrm{SD}$ ). Six subjects participated in experiments 4 and 5 (five females; experiment 4: age, $25 \pm 3.9 \mathrm{SD}$; experiment 5: age, $28 \pm 1.9 \mathrm{SD}$ ). All subjects gave written informed consent. This study was approved by the ethics committee of the University of Goettingen, and we conform to the Declaration of Helsinki.

Measurement of motor cortical excitability. To monitor tDCS- and PASdriven changes of motor cortex excitability, muscle-evoked potentials (MEPs) of the right abductor digiti minimi muscle (ADM) were recorded after stimulation of their motor cortical representational fields by single-pulse TMS. These were induced using a Magstim 200 magnetic stimulator (Magstim Company, Dyfed, UK) and a figure-of-eight magnetic coil (diameter of one winding, $70 \mathrm{~mm}$; peak magnetic field, 2.2 tesla). The coil was held tangentially to the skull, with the handle pointing backwards and laterally at $45^{\circ}$ from midline. The optimum coil position was defined as the site where stimulation resulted consistently in the largest MEP. The surface EMG was recorded from the right ADM by use of $\mathrm{Ag}-\mathrm{AgCl}$ electrodes in a belly-tendon montage. The signals were amplified and filtered with a time constant of $10 \mathrm{~ms}$ and a low-pass filter of $2.5 \mathrm{kHz}$. Signals were then digitized at an analog-to-digital rate of $5 \mathrm{kHz}$ and relayed into a laboratory computer using the Signal 1.62 software (Cambridge Electronics Design, Cambridge, UK) and conventional averaging software. The intensity of the stimulator output was adjusted for baseline recording so that the average stimulus led to an MEP of $\sim 1 \mathrm{mV}$.

Direct current stimulation of the motor cortex. Direct currents were transferred by a pair of saline-soaked surface sponge electrodes and delivered by a specially developed, battery-driven, constant-current stimulator (Schneider Electronic, Gleichen, Germany) with a maximum output of $10 \mathrm{~mA}$. In each experiment, the motor-cortical electrode was fixed over the cortical representational field of the right ADM as identified by TMS and the other electrode contralaterally above the right orbit. The currents flowed continuously for $7 \mathrm{~min}$ before or during PAS in experiments 2, 3, and 5 to induce excitability shifts lasting for some minutes. In experiment 4, anodal or cathodal tDCS was administered for $15 \mathrm{~min}$. In former experiments, this stimulation duration generates after-effects lasting for $\sim 5-10 \mathrm{~min}$ ( $7 \mathrm{~min} \mathrm{tDCS}$ ) or $1 \mathrm{~h} \mathrm{(15} \mathrm{min} \mathrm{tDCS)} \mathrm{after} \mathrm{the} \mathrm{end}$ of stimulation (Nitsche and Paulus, 2001; Nitsche et al., 2003a). Motor cortex electrode size was $35 \mathrm{~cm}^{2}$ and current strength was $1 \mathrm{~mA}$ (current density, $\sim 0.03 \mathrm{~mA} / \mathrm{cm}^{2}$ ).

PAS. PAS was conducted according to the paradigm first described by Stefan et al. (2000). Only excitability-enhancing PAS protocols were used in this experiment. Peripheral nerve stimulation was performed on the right ulnar nerve at the wrist with an intensity $300 \%$ above sensory threshold with a Digitimer (Welwyn Garden City, UK) D185 stimulator. A single TMS pulse was delivered over the left motor cortical representation of the right ADM $25 \mathrm{~ms}$ after peripheral stimulation. TMS intensity was determined to elicit an MEP amplitude size of $\sim 1 \mathrm{mV}$. PAS was performed every $20 \mathrm{~s}$ for 7, 15, or $30 \mathrm{~min}$ in the different experiments.

Experimental procedures general experimental course. Each experiment was conducted in a repeated-measurement design. The order of the single sessions within an experimental protocol was randomized between subjects. A break of $\geq 1$ week was obligatory between single experimental sessions to avoid interference effects.
Subjects were seated in a reclining chair. After fixing the surface EMG electrodes above the right ADM, its left motor cortical representational field was identified by use of TMS (coil position that leads to the largest MEPs of ADM). Then, the TMS intensity resulting in MEP amplitudes of $\sim 1 \mathrm{mV}$ was established. For peripheral ulnar nerve stimulation, the optimum electrode position was identified and the electrode was fixed at this position with adhesive tape. Stimulation intensity was increased stepwise from a clear subthreshold level until the subjects felt a physical sensation. The lowest stimulation intensity that elicited a reliable sensation was defined as the threshold. Next, a baseline TMS measure was conducted to document pre-PAS motor cortex excitability. Twenty TMSs were recorded (frequency, $0.25 \mathrm{~Hz}$ ). Afterward, PAS/tDCS was performed. After PAS, TMS measures of excitability (baseline-intensity, 15 stimuli per time bin at $0.25 \mathrm{~Hz}$ ) were performed every 5 min for 30 min and then every $30 \mathrm{~min}$ for up to $90 \mathrm{~min}$ after stimulation. TMS intensity was held constant throughout the experiment.

Adjustment of PAS duration (experiment 1). This experiment was conducted to identify suitable PAS durations that would induce reliable short- or long-lasting excitability enhancements of the motor cortex. Because the original stimulation protocol (Stefan et al., 2000) lasts for 30 min and induces excitability changes stable for $>1 \mathrm{~h}$, it is not well suited to be combined with $\mathrm{tDCS}$. We therefore reduced PAS duration systematically from $30 \mathrm{~min}$, as described in the original protocol, to 15 and 7 min. After-effects were monitored up to 90 min after the end of PAS.

Modification of short-lasting PAS-induced excitability changes by previous administration of tDCS (experiment 2). This experiment was conducted for the 7 min PAS condition, which was shown to induce relatively short-lived, but still significant, excitability enhancements in experiment 1 . Anodal and cathodal tDCS were also administered for 7 min to induce short-lasting after-effects. Four experimental sessions were conducted for each subject: anodal tDCS followed by PAS or without PAS and cathodal tDCS followed by PAS or without PAS. DCS electrodes were fixed on the subject's head after identification of the left motor cortical ADM representation and baseline TMS measures, as described above. The PAS was administered immediately after the end of tDCS and removal of the respective electrodes. The after-effects of stimulation were measured as described above for $90 \mathrm{~min}$ after the end of intervention.

Modification of short-lasting PAS-induced excitability changes by simultaneous administration of tDCS (experiment 3 ). The design of this experiment was identical to experiment 2 , with the exception that anodal or cathodal tDCS was performed not before but during PAS in this condition. Consequently, TMS measures had to be performed through the tDCS electrodes, thus these were fixed after determination of the cortical ADM representation onto the head of the subjects and kept there for the whole course of the experiment. After-effects were measured for $90 \mathrm{~min}$ after intervention.

Modification of long-lasting PAS-induced excitability changes by simultaneous administration of $t D C S$ (experiment 4). The protocol was identical to that of experiment 3, except for the fact that here 15 min PAS (which induces excitability enhancements stable for a longer duration than 7 min PAS) and 15 min anodal tDCS were combined. PAS was conducted with and without tDCS in different sessions separated by $\geq 1$ week. After-effects were monitored for $90 \mathrm{~min}$ after stimulation as described above and additionally on the evening of the stimulation day, as well as three times the day after stimulation (morning, noon, evening) for the combined PAS/tDCS protocol. TMS coil position, tDCS electrode position, and ADM recording electrode position were marked with a waterproof pen to guarantee constant electrode positions throughout the experiment.

Modification of tDCS-induced excitability changes by simultaneous TMS (experiment 5). In this control experiment, we tested whether simultaneous administration of tDCS and single-pulse TMS (20 s interstimulus interval) results in excitability changes comparable to those of tDCS with simultaneous PAS. The experimental protocol resembles experiment 3, anodal tDCS combined with PAS (and the respective tDCS-only measure), with the exception that the peripheral stimulus was omitted. Aftereffects were measured for up to 90 min after stimulation.

Calculations and statistics. MEP amplitude means were calculated for 
Table 1. Results of the ANOVAs conducted for the different experiments

\begin{tabular}{|c|c|c|c|}
\hline Experiments & df & Fvalue & $p$ value \\
\hline \multicolumn{4}{|l|}{ PAS only (experiment 1) } \\
\hline PAS & 2 & 27.487 & $<0.001^{*}$ \\
\hline Time course & 9 & 59.721 & $<0.001^{*}$ \\
\hline PAS $\times$ time course & 18 & 7.743 & $<0.001^{*}$ \\
\hline \multicolumn{4}{|l|}{ tDCS only } \\
\hline $\mathrm{tDCS}$ & 1 & 294.420 & $<0.001^{*}$ \\
\hline Time course & 9 & 0.885 & 0.568 \\
\hline $\mathrm{tDCS} \times$ time course & 9 & 43.447 & $<0.001^{*}$ \\
\hline \multicolumn{4}{|c|}{ Short-lasting PAS and tDCS (experiments 2,3) } \\
\hline Stimulation & 6 & 37.868 & $<0.001^{*}$ \\
\hline Time course & 9 & 24.187 & $<0.001^{*}$ \\
\hline Stimulation $\times$ time course & 54 & 14.043 & $<0.001^{*}$ \\
\hline \multicolumn{4}{|c|}{ Long-lasting PAS and tDCS (experiment 4) } \\
\hline Stimulation & 1 & 38.826 & $0.002 *$ \\
\hline Time course & 9 & 8.726 & $<0.001^{*}$ \\
\hline Stimulation $\times$ time course & 9 & 8.814 & $<0.001^{*}$ \\
\hline \multicolumn{4}{|c|}{ Single pulse TMS and tDCS (experiment 5) } \\
\hline Stimulation & 1 & 0.912 & 0.383 \\
\hline Time course & 9 & 19.994 & $<0.001^{*}$ \\
\hline Stimulation $\times$ time course & 9 & 0.990 & 0.462 \\
\hline
\end{tabular}

The asterisks mark significant main effects and interactions.

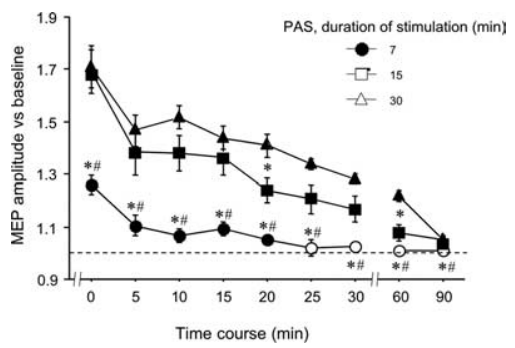

Figure 1. Dose-dependent effects of facilitatory PAS on motor cortex excitability. PAS duration was 7, 15, or $30 \mathrm{~min}$. The time course of baseline-standardized MEP amplitudes elicited by single-pulse TMS after PAS is depicted. Each protocol resulted in significant excitability enhancement compared with baseline (significant deviations from baseline, filled symbols) (paired two-tailed ttests; level of significance, 0.05 ). However, the 7 min PAS resulted in smaller and shorter-lasting excitability enhancements than 15 and 30 min PASs (significant differences between 7 and 15 min PASs, asterisks; between 7 and 30 min PASs, pound symbols) (paired two-tailed $t$ tests; level of significance, 0.05 ). Moreover, the 30 min PAS resulted in slightly larger excitability enhancements than the $15 \mathrm{~min}$ PAS (significant differences between 15 and 30 min PAS, asterisks) (paired two-tailed $t$ tests; level of significance, 0.05 ). The error bars indicate SEM. The dashed line indicates the baseline MEP amplitude.

each time bin covering the preintervention baseline (20 stimuli) and postintervention time points (15 stimuli). Postintervention MEP amplitude means were normalized to preintervention baselines. For experiment 1 , a repeated-measures ANOVA was calculated; the independent variables were the PAS duration and time course, and the dependent variable was the MEP amplitude. Student's $t$ tests (paired samples; twotailed; level of significance $<0.05$ ) were performed to determine whether the MEP amplitudes before and after PAS differed for each time bin and whether those differences depended on PAS duration.

Similarly, with regard to experiments 2, 3, 4, and 5, repeated-measure ANOVAs (independent variables, time course and stimulation condition; dependent variable, MEP amplitude) were calculated, then Student's $t$ tests (paired samples; two-tailed; level of significance $<0.05$ ) were performed to determine whether the MEP amplitudes before and after intervention differed for each time bin and whether those differences depended on the type of intervention. Additionally, baseline MEPamplitudes were controlled within each experiment for differences between interventions by Student's $t$ tests (paired samples; two-tailed; level of significance $<0.05)$.

\section{Results \\ Adjustment of PAS duration (experiment 1)}

The results of the ANOVA display significant main effects of time and PAS protocol because of the fact that PAS induced significant enhancements of MEP amplitudes in all stimulation conditions and that these enhancements diminished over time (Table 1, Fig. 1). The significant interaction of PAS protocol and time course reflects the fact that prolonged PAS induced stronger and longer-lasting excitability enhancements than shorter protocols. Whereas 7 min PAS induced an initial increase in the MEP amplitude of $130 \%$ of baseline measures, which was diminished to a $10 \%$ increase after a few minutes and went back to baseline $25 \mathrm{~min}$ after the end of stimulation, 15 and $30 \mathrm{~min}$ PAS resulted in an MEP amplitude enhancement of $\sim 150 \%$ relative to baseline, which was significant up to $90 \mathrm{~min}$ after stimulation (Fig. 1). Baseline MEPs were identical in all conditions. Thus, the efficacy of PAS in inducing motor cortex excitability enhancements follows a dose-response relationship. Longer-lasting PAS induced increased effects compared with shorter protocols. For the PAS protocol, which includes $30 \mathrm{~min}$ stimulation, the results are comparable with those of former studies (Stefan et al., 2000).

\section{Modification of short-lasting PAS-induced excitability changes by previous or simultaneous administration of tDCS (experiments 2 and 3 )}

For the tDCS-only condition, the ANOVA reveals a significant main effect of tDCS and a significant interaction of tDCS $\times$ time course (Table 1). This was caused by polarity-specific MEP increases or reductions observable for $\sim 60 \mathrm{~min}$ after the end of tDCS. Anodal tDCS increased, whereas cathodal tDCS diminished motor cortex excitability (Fig. 2). As shown by the results of the ANOVA conducted for all stimulation conditions, the main effects of stimulation and time course and the respective interaction between these factors were significant (Table 1). When excitability-enhancing anodal tDCS was applied before PAS, the resulting excitability enhancement was stronger and longer lasting compared with the application of PAS or tDCS alone. Cathodal tDCS administered before PAS, however, resulted in a slight diminution of the MEP amplitude after PAS and thus antagonized the effect of PAS alone (Fig. 3a). However, simultaneous application of PAS and tDCS resulted in an excitability diminution in the case of anodal tDCS, whereas simultaneous cathodal tDCS and PAS induced a prolonged excitability enhancement (Fig. 3b). Baseline MEPs were identical in all conditions. Seven minute anodal or cathodal tDCS alone resulted in motor cortex excitability enhancements and reductions similar in magnitude to former studies of our group (Nitsche and Paulus, 2001; Nitsche et al., 2003a). However, the respective effects were longer-lasting, most probably resulting from group specifics, because substantial interindividual variability of the proneness of subjects to react to tDCS was documented before (Nitsche and Paulus, 2001; Nitsche et al., 2003a, 2004a,b). 


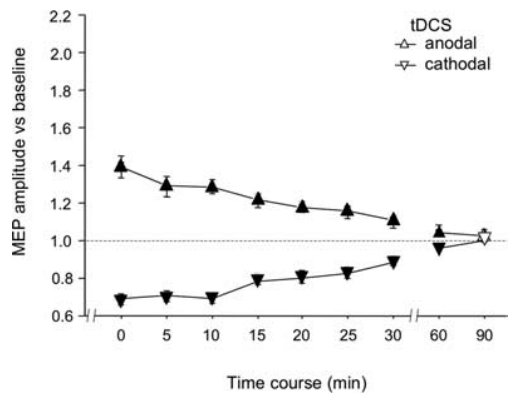

Figure 2. Effect of tDCS alone on motor cortex excitability. Anodal or cathodal tDCS was administered for $7 \mathrm{~min}$. The time course of baseline-standardized MEP amplitudes elicited by single-pulse TMS after TDCS is depicted. Anodal tDCS enhanced, whereas cathodal tDCS diminished, MEP amplitudes for $60 \mathrm{~min}$ relative to baseline. Filled symbols indicate significant differences between baselines and post-tDCS MEPs (paired two-tailed $t$ tests; level of significance, 0.05). Error bars indicate SEM. The dashed line indicates the baseline MEP amplitude.

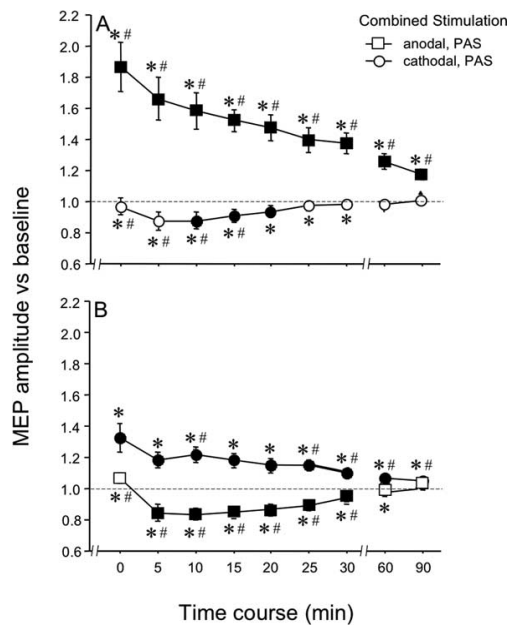

Figure 3. Effect of combined tDCS and PAS on motor cortex excitability. The time course of baseline-standardized MEP amplitudes elicited by single-pulse TMS after PAS combined with tDCS (20 PASs; 7 min tDCS) is depicted. $A$, When tDCS was applied before PAS, combined anodal tDCS and PAS resulted in an excitability enhancement exceeding in magnitude and duration than the excitability change elicited by anodal tDCS or PAS alone, whereas cathodal tDCS combined with PAS resulted in a minor excitability reduction that, however, was smaller than the excitability diminution achieved by cathodal tDCS alone. $\boldsymbol{B}$, When PAS and tDCS were administered simultaneously, anodal tDCS and PAS resulted in a prolonged excitability diminution, whereas cathodal tDCS applied together with PAS enhanced excitability. Filled symbols indicate significant deviations of the postintervention MEP amplitudes relative to baseline MEPs, and asterisks indicate significant differences to tDCS of the same polarity given alone and pound symbols to PAS alone (paired two-tailed $t$ tests; level of significance, 0.05 ). Error bars indicate SEM. The dashed line indicates the baseline MEP amplitude.

\section{Modification of long-lasting PAS-induced excitability changes by simultaneous administration of $\mathrm{tDCS}$ (experiment 4)}

The results of the respective ANOVA display significant main effects of time course and stimulation as well as a significant interaction between these factors (Table 1). This is because of a significant excitability enhancement induced by 15 min PAS, which, however, is turned into inhibition if combined with simultaneous anodal tDCS (Fig. 4). Interestingly, this diminution of MEP amplitudes remains until the noon after the stimulation day and thus for $\sim 24 \mathrm{~h}$ after stimulation. Because prolonged tDCS and PAS resulted in identically directed homeostatic plasticity changes, compared with the shorter-lasting protocols, the stability of the neuroplastic excitability changes seems not to be

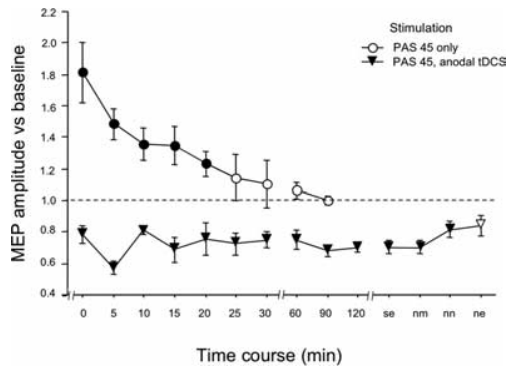

Figure 4. Prolonged tDCS/PAS enhances the duration of homeostatic plasticity. The time course of baseline-standardized MEP amplitudes elicited by single-pulse TMS after PAS combined with tDCS (45 PASs; 15 min anodal tDCS) is depicted. Simultaneously administered anodal tDCS converts the excitability-enhancing effect of PAS into inhibition. This effect is directed identically to the shorter stimulation protocol, but the excitability diminution is significantly prolonged up to midday after the day of stimulation. Filled symbols indicate significant differences relative to baseline MEPs. Error bars indicate SEM. The dashed line indicates the baseline MEP amplitude. se, Same evening; nm, next morning; nn, next noon; ne, next evening.

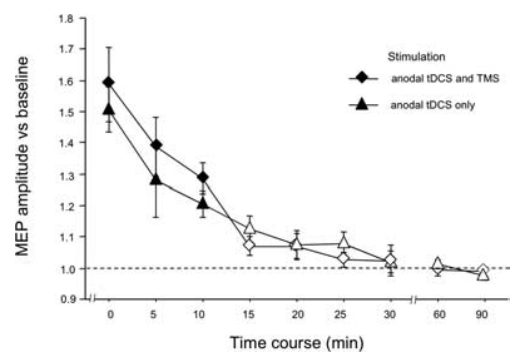

Figure 5. TMS alone does not induce homeostatic alterations of anodal tDCS-elicited plasticity. The time course of baseline-standardized MEP amplitudes elicited by single-pulse TMS after anodal tDCS only for $7 \mathrm{~min}$ and anodal tDCS combined with TMSs (every $20 \mathrm{~s}$, thus resembling the TMS frequency of the respective PAS protocol) is depicted. Both protocols resulted in identical excitability enhancements. Filled symbols indicate significant differences relative to baseline MEPs. Error bars indicate SEM. The dashed line indicates the baseline MEP amplitude.

critical. Baseline MEP amplitudes did not differ between both stimulation conditions.

\section{Modification of tDCS-induced excitability changes by simultaneous TMS (experiment 5)}

Here, the ANOVA reveals a significant main effect of time course, but the main effect of stimulation as well as the interaction between both variables turned out to be not significant (Table 1). This is because of an identical MEP amplitude enhancement accomplished by anodal tDCS ( $7 \mathrm{~min}$ ) alone and the combination of tDCS with TMS (single pulse every $20 \mathrm{~s}$ ), which is significant for up to $10 \mathrm{~min}$ after the end of stimulation (Fig. 5). Baseline MEP amplitudes are identical for both stimulation conditions.

Thus, TMS alone does not suffice to induce homeostatic plasticity when administered simultaneously with tDCS. We conclude that the homeostatic effect observed after simultaneous tDCS/PAS is indeed caused by an influence of tDCS on associative synapse-specific plasticity, as induced by PAS.

\section{Discussion}

Homeostatic plasticity (i.e., the dependency of the amount and direction of the obtainable plasticity from the history of activity of a neuronal network) is increasingly recognized as regulatory mechanism for keeping neuronal modifications within a reasonable physiological range. Here, we demonstrate an acute homeostatic effect of global network level of activity on synapse-specific 
plasticity, which only occurs if both plasticity-inducing protocols are applied simultaneously.

PAS, which induces a motor cortex excitability enhancement when applied alone, resulted in an excitability diminution when combined simultaneously with excitability-enhancing tDCS. If excitability-reducing tDCS was coadministered, it increased and prolonged the PAS-induced excitability enhancement. Control experiments revealed that (1) this effect indeed depends on the interaction of tDCS and PAS, because administering solely the TMS stimulus of the PAS protocol did not influence tDCSinduced plasticity, and (2) this homeostatic effect is not restricted to plasticity protocols inducing short-lasting plasticity, because prolonged tDCS and PAS protocols resulted in identically directed effects. Conversely, tDCS applied before PAS did modify the PAS-induced excitability changes synergistically. Thus, the effect of background activity/excitability on associative plasticity critically depends on their temporal relationship: whereas previous background activity modulates associative plasticity synergistically, simultaneous modification of background and associative, synapse-specific plasticity modifies the latter antagonistically and thus in a homeostatic manner.

Traditionally, homeostatic plasticity is thought to act within an extended time scale. In animal experiments, enhanced or reduced basal neuronal network activity held constant for days or weeks influences the effects of acute plasticity-inducing protocols homeostatically (Burrone and Murthy, 2003; Turrigiano and Nelson, 2004). However, homeostatic mechanisms can work on a much shorter time scale in animals (van Welie et al., 2004) and humans (Iyer et al., 2003; Lang et al. 2004; Siebner et al., 2004). In humans, preconditioning of the motor cortex with excitabilitymodifying external stimulation (rTMS or tDCS) determines the direction of neuroplastic excitability changes induced by a succeeding stimulation protocol homeostatically. In contrast to these studies, in which stimulation protocols were applied that involve the whole cortical area stimulated, the current experiments probed the effect of a global network plasticity change on synaptically restricted associative plasticity. Our results differ clearly from those of the former studies: preconditioning of associative plasticity with global excitability-enhancing tDCS boosted the efficacy of the subsequent PAS protocol to produce excitatory neuroplasticity, whereas excitability-diminishing tDCS reduced it. However, simultaneous global network modification and associative plasticity induction reversed these effects (i.e., induced homeostatic plasticity).

The differential effects of preconditioning versus simultaneous network modification on associative plasticity give rise to two main questions: why does this kind of homeostatic plasticity follow a different time rule than homeostatic mechanisms induced by other stimulation protocols, and what is the underlying physiological mechanism? In recent studies in humans, consecutively administered neuroplasticity-inducing stimulation protocols induced homeostatic effects (Iyer et al., 2003; Lang et al., 2004; Siebner et al., 2004). In two of these studies, the preconditioning stimulation protocol was identical to the current one (i.e., tDCS). The main difference lies in the conditioning protocol, which was rTMS in the former studies but PAS in the current one. PAS induces timing-dependent, synapse-specific neuroplasticity. rTMS, in contrast, induces neuroplasticity, which may not be restricted to specific synaptic connections. In doing so, rTMS might induce patterns of neuroplasticity similar to that of tDCS. Thus, subsequent neuroplasticity-inducing protocols that have similar effects on global network plasticity may work antagonis- tically to keep global network function within a physiological range.

However, synapse-specific and thus spatially restricted neuroplasticity, as induced by PAS, might not suffice to induce counteracting homeostatic mechanisms. It thus might profit from previous global excitability-enhancing neuroplasticity and be diminished by antecedent global excitability-diminishing neuroplasticity. However, simultaneous administration of tDCS and PAS did induce homeostatic plasticity. Such a homeostatic mechanism makes sense for optimizing information processing, because it would strengthen synapse-specific plasticity in a silent network while suppressing it during high-level general network activity. So what is the physiological difference between succeeding and simultaneous $\mathrm{tDCS} / \mathrm{PAS}$ protocols causing these dissociating effects? Although knowledge about the mechanisms responsible for the induction of neuroplasticity by tDCS and PAS is limited, it is known that ion channels are involved in the induction of plasticity and NMDA receptors in the stabilization of the after-effects of both plasticity-inducing stimulation protocols (Liebetanz et al., 2002; Stefan et al., 2002; Nitsche et al., 2003b). Because it has been shown that intraneuronal calcium concentration regulates homeostatic plasticity (Misonou et al., 2004), this could be a candidate mechanism. However, alternative processes cannot be ruled out by the results of our experiments. Thus, the specific mechanisms of action have to be explored in future studies.

Associative plasticity is thought to be a neurophysiological correlate of learning and memory formation in humans and animals (Rioult-Pedotti et al., 1998, 2000; Ziemann et al., 2004; Stefan et al., 2006). Recently, motor cortex tDCS has been demonstrated to improve motor and visuomotor coordination learning in humans (Nitsche et al., 2003c; Antal et al., 2004). Given the results of our study, an optimum effect on boosting learningrelated associative plasticity should be achieved by excitabilityenhancing anodal tDCS administered before the actual learning process or excitability-reducing cathodal tDCS during learning. However, if applied during learning only anodal but not cathodal tDCS improved learning substantially in both of the abovementioned studies. Furthermore, if applied before the behavioral task, anodal and cathodal tDCS did not modify performance in one of the paradigms and had only minor effects in the other (unpublished results of our group). These results are in clear opposition to the effects of tDCS on PAS. One explanation might be that PAS indeed resembles one important synaptic derivate of learning processes, but the frequency of application of associative stimuli is much too low to realistically represent neuronal activity during complex learning processes, as tested in the abovementioned studies. In this way, PAS might represent an activity insufficient on its own to serve as a salient signal against increased actual background activity and thus might be toned down. This argument does not rule out that PAS resembles neuronal derivates of learning and memory formation in principle. The studies of Ziemann et al. (2004) and Stefan et al. (2006) have demonstrated elegantly its involvement, but the results of our study put into question whether the specific low-frequency PAS protocol applied here is sufficient to mimic learning processes. Alternatively, it might be argued that homeostatic effects of tDCS on motor learning and PAS-induced plasticity dissociate on a time scale: in another study of our group (Lang et al., 2003), it was shown that anodal tDCS administered during motor learning selectively impaired recall of the learned sequence. This result might argue for a delayed homeostatic effect of background excitability enhancement on memory consolidation. Additional 
studies are needed to clarify the specific relationship of PAS and learning to a greater extent.

In a recent study, Baumgartner et al. (2007) have demonstrated a relevant impact of emotional state and arousal on the size of MEP amplitudes. We think that we can exclude a relevant influence of these parameters or the results of our study for the following reasons. First, in a recent study, tDCS of the dorsolateral prefrontal cortex, which modifies mood in depressed subjects, did not influence emotional state and arousal in healthy subjects (our unpublished observations). Second, modulation of general arousal or emotional state during the course of the experiment would result in similar MEP enhancements independent of the stimulation protocol used. On the contrary, our results strongly depend on the specific stimulation protocol. Moreover, because we used a randomized repeated-measurement design, any change of arousal or emotional state caused by, for example, the first participation of one subject in this kind of experiment would be randomly assigned to the specific experimental conditions, and thus its systematic contribution to the results is improbable. However, the results of the study of Baumgartner et al. (2007) point to the general fact that it is important to control for emotional state and arousal during the conduction of experiments using MEPs as dependent variables.

Together, the results of our study present a new homeostatic plasticity mechanism, which only applies when associative plasticity is induced during enhanced or reduced background activity. This kind of homeostatic plasticity thus clearly differs from other metaplastic mechanisms described so far and sheds light on the fact that multiple homeostatic mechanisms might exist that differ because of the kinds of plasticity induced as well as because of the time course of plasticity induction. Future studies should focus on the underlying mechanisms of these different kinds of metaplasticity and explore their functional relevance.

\section{References}

Abraham WC, Tate WP (1997) Metaplasticity: a new vista across the field of synaptic plasticity. Prog Neurobiol 52:303-323.

Antal A, Nitsche MA, Kinsces TZ, Kruse W, Hoffmann K-P, Paulus W (2004) Facilitation of visuo-motor learning by transcranial direct current stimulation of the motor and extrastriate visual areas in humans. Eur J Neurosci 19:2888-2892.

Baumgartner T, Willi M, Jancke L (2007) Modulation of corticospinal activity by strong emotions evoked by pictures and classical music: a transcranial magnetic stimulation study. NeuroReport 18:261-265.

Bienenstock EL, Cooper LN, Munro PW (1982) Theory for the development of neuron selectivity: orientation specificity and binocular interaction in visual cortex. J Neurosci 2:32-48.

Burrone J, Murthy VN (2003) Synaptic gain control and homeostasis. Curr Opin Neurobiol 13:560-567.

Iyer MB, Schleper N, Wassermann EM (2003) Priming stimulation enhances the depressant effect of low-frequency repetitive transcranial magnetic stimulation. J Neurosci 23:10867-10872.

Lang N, Nitsche MA, Sommer M, Tergau F, Paulus W (2003) Modulation of motor consolidation by external DC stimulation. Suppl Clin Neurophysiol 56:277-281.

Lang N, Siebner HR, Ernst D, Nitsche MA, Paulus W, Lemon RN, Rothwell JC (2004) Preconditioning with transcranial direct current stimulation sensitizes the motor cortex to rapid-rate transcranial magnetic stimulation and controls the direction of after-effects. Biol Psychiatry 56:634-639.
Li H, Weiss SR, Chuang DM, Post RM, Rogawski MA (1998) Bidirectional synaptic plasticity in the rat basolateral amygdala: characterization of an activity-dependent switch sensitive to the presynaptic metabotropic glutamate receptor antagonist $2 \mathrm{~S} \alpha$-ethylglutamic acid. J Neurosci 18:1662-1670.

Liebetanz D, Nitsche MA, Tergau F, Paulus W (2002) Pharmacological approach to synaptic and membrane mechanisms of DC-induced neuroplasticity in man. Brain 125:2238-2247.

Misonou H, Mohapatra DP, Park EW, Leung V, Zhen D, Misonou K, Anderson AE, Trimmer JS (2004) Regulation of ion channel localization and phosphorylation by neuronal activity. Nat Neurosci 7:711-718.

Nitsche MA, Paulus W (2001) Sustained excitability elevations induced by transcranial DC motor cortex stimulation in humans. Neurology 57:1899-1901.

Nitsche MA, Nitsche MS, Klein CC, Tergau F, Rothwell JC, Paulus W (2003a) Level of action of cathodal DC polarisation induced inhibition of the human motor cortex. Clin Neurophysiol 114:600-604.

Nitsche MA, Fricke K, Henschke U, Schlitterlau A, Liebetanz D, Lang N, Henning S, Tergau F, Paulus W (2003b) Pharmacological modulation of cortical excitability shifts induced by transcranial DC stimulation. J Physiol (Lond) 553:293-301.

Nitsche MA, Schauenburg A, Lang N, Liebetanz D, Exner C, Paulus W, Tergau F (2003c) Facilitation of implicit motor learning by weak transcranial direct current stimulation of the primary motor cortex in the human. J Cog Neurosci 15:619-626.

Nitsche MA, Grundey J, Liebetanz D, Lang N, Tergau F, Paulus W (2004a) Catecholaminergic consolidation of motor cortex plasticity in humans. Cereb Cortex 14:1240-1245.

Nitsche MA, Jaussi W, Liebetanz D, Lang N, Tergau F, Paulus W (2004b) Consolidation of externally induced human motor cortical neuroplasticity by d-cycloserine. Neuropsychopharmacology 29:1573-1578.

Rioult-Pedotti MS, Friedman D, Hess G, Donoghue JP (1998) Strengthening of horizontal cortical connections following skill learning. Nat Neurosci 1:230-234.

Rioult-Pedotti MS, Friedman D, Hess G, Donoghue JP (2000) Learninginduced LTP in neocortex. Science 290:533-536.

Siebner HR, Lang N, Rizzo V, Nitsche MA, Paulus W, Lemon RN, Rothwell JC (2004) Preconditioning of low-frequency repetitive transcranial magnetic stimulation with transcranial direct current stimulation: evidence for homeostatic plasticity in the human motor cortex. J Neurosci 24:3379-3385.

Stefan K, Kunesch E, Cohen LG, Benecke R, Classen J (2000) Induction of plasticity in the human motor cortex by paired associative stimulation. Brain 123:572-584.

Stefan K, Kunesch E, Benecke R, Cohen LG, Classen J (2002) Mechanisms of enhancement of human motor cortex excitability induced by interventional paired associative stimulation. J Physiol (Lond) 543:699-708.

Stefan K, Wycislo M, Gentner R, Schramm A, Naumann M, Reiners K, Classen J (2006) Temporary occlusion of associative motor cortical plasticity by prior dynamic motor training. Cereb Cortex 16:376-385.

Turrigiano GG, Nelson SB (2004) Homeostatic plasticity in the developing nervous system. Nat Rev Neurosci 5:97-107.

Turrigiano GG, Leslie KR, Desai NS, Rutherford LC, Nelson SB (1998) Activity-dependent scaling of quantal amplitude in neocortical neurons. Nature 391:892-896.

van Welie I, van Hooft JA, Wadman WJ (2004) Homeostatic scaling of neuronal excitability by synaptic modulation of somatic hyperpolarizationactivated Ich channels. Proc Natl Acad Sci USA 101:5123-5128.

Ziemann U, Ilic TV, Pauli C, Meintzschel F, Ruge D (2004) Learning modifies subsequent induction of long-term potentiation-like and long-term depression-like plasticity in human motor cortex. J Neurosci 24:16661672. 


\subsection{No Major influence of homeostatic plasticity in complex motor learning in humans}

The present study was conducted to investigate if and how homeostatic plasticity regulates real learning process. For serial reaction time task (SRTT), an implicit motor learning paradigm, it has been demonstrated that motor learning can be enhanced by simultaneous application of excitability-enhancing anodal tDCS during learning process (Nitsche et al., 2003c). Here we aimed to test the functional significance of homeostatic plasticity on cognitive functions since learning processes should be improved by keeping the cortical plastic changes within an optimal range in accordance with the homeoplastic regulations (Abraham, 1999). Due to the homeostatic paradigm that the history of activation should homeostatically affect actual plasticity, tDCS was applied before the task started. We also combined the partial NMDA receptor agonist D-Cycloserine as an additional modulating factor to test whether it further enhances the influence of homeostatic plasticity on the learning process, since it was argued that pharmacological enhancement of task-related active NMDA-receptors in otherwise silent networks could further increase a positive effect of homeostatic plasticity on learning. As the results showed, the homeostatic mechanisms apply only when anodal tDCS was combined with D-Cycloserine, namely, it impaired motor learning. This suggests a minor effect of homeoplastic mecahnisms on cortical excitability on actual learning process in humans, which might be regulated by a far more complicated mechanism compared to the simple neurophysiological modulation of cortical plasticity. 


\title{
No Major influence of homeostatic plasticity in complex motor learning in humans
}

Min-Fang Kuo, MD, Mandy Unger, Andrea Antal, PhD, David Liebetanz, MD, Nicolas Lang, MD, Frithjof Tergau, MD, Walter Paulus, MD, Michael A. Nitsche, MD

Department of Clinical Neurophysiology, University of Göttingen, Robert Koch Str. 40, 37099 Göttingen, Germany

\begin{abstract}
Neuroplasticity is the adaptive modification of network connectivity due to environmental demands and has been identified as a major physiological correlate of learning in animals and humans. Since unrestricted neuroplastic modifications of network connectivity will result in a destabilization of the system, metaplastic modification rules have been proposed and identified for keeping plastic connectivity changes within a useful dynamic range. Hereby, the modification threshold to achieve neuroplastic synaptic strengthening or weakening is suggested to depend on the history of activity of the respective neurons. However, the relevance of metaplasticity for actual learning processes has not been tested so far directly. Here, we reduced or enhanced motor cortex activity and excitability before performance of the serial reaction time task (SRTT), a sequential motor learning paradigm, and a simple reaction time task (RTT) by transcranial direct current stimulation (tDCS). If homeostatic rules apply, excitability-diminishing cathodal tDCS should improve the subsequent task performance, especially if combined with the partial NMDA receptor-agonist D-Cycloserine, which selectively enhances efficacy of active receptors, while excitability-enhancing anodal tDCS should reduce it. However, only the results for anodal tDCS, when combined with DCycloserine, were in accordance with the rules of homeostatic plasticity. We conclude that homeostatic plasticity, as tested here, has only a minor influence on motor learning and performance.
\end{abstract}




\section{Introduction}

Motor skill acquisition involves the strengthening of synaptic connections, probably reflecting NMDA receptor-dependent long-term potentiation (LTP), which is an important correlate of motor learning in animals in vivo (Rioult-Pedotti et al., 2000). Recently it was demonstrated that LTP-like processes are also involved in motor learning in humans (Stefan et al. 2006, Ziemann et al., 2004). These neuroplastic modifications take place at least in part in the primary motor cortex (Müllbacher et al. 2002, Nitsche et al. 2003a).

Within the last years it became increasingly clear that neuroplasticity has to be controlled for, because unrestricted plastic changes will result in massive modifications of neuronal networks, which will destabilize the system and prevent further dynamic adaptive modifications. Therefore, metaplastic rules were introduced first in simulated neuronal networks, but also experimentally revealed in animal and human studies (for an overview see Abbott and Nelson 2000, Abraham and Tate 1997, Turrigiano and Nelson 2000). One prominent regulatory mechanism, the Bienenstock-Cooper-Munro rule (1982), postulates the dependency of subsequent neuroplastic excitability enhancements or reductions on the history of activation: A previous high level of activity will shift ongoing neuroplastic events in the direction of inhibition, while a history of low activation will favor facilitation, both due to a sliding synaptic modification threshold. While the exact mechanism of this dynamic control of neuroplasticity is still subject of debate, its principle applicability has been demonstrated in human experiments: Priming with excitability-enhancing high-frequency repetitive transcranial magnetic stimulation (rTMS) of the motor cortex increased the excitabilityreducing effects of a subsequent low-frequency rTMS protocol (Iyer et al. 2003). Similarly, motor cortex excitability and activity diminution by cathodal transcranial direct current stimulation (tDCS) caused high- and low-frequency rTMS protocols, which were without an effect on excitability, when given alone, to increase excitability, while excitability-enhancing anodal tDCS had reverse effects (Lang et al. 2004, Siebner et al. 2004).

However, the functional relevance of metaplasticity for learning processes has not been tested experimentally so far. Thus the aim of this study was to explore the effect of a modulation of the history of activation and excitability of the primary motor cortex on subsequent motor learning. Anodal or cathodal tDCS was administered before performance of a sequential motor learning task, the serial reaction time task (SRTT, Nissen and Bullemer 1987). tDCS induces prolonged subthreshold modifications of the resting membrane potential, altering 
neuronal activity and excitability. Anodal tDCS enhances, and cathodal stimulation reduces activity (Nitsche and Paulus 2000, 2001, Nitsche et al. 2003b). The after-effects are NMDA receptor-dependent (Liebetanz et al. 2002, Nitsche et al. 2003c, 2004). Anodal tDCS of the primary motor cortex had already been shown to improve sequence acquisition, if applied during SRTT performance, in a foregoing study (Nitsche et al. 2003a), most probably by bringing learning-related synapses nearer to their modification threshold.

Given that metaplasticity influences motor learning, we hypothesized that anodal tDCS applied prior learning would decrease performance due to diminished strengthening of learning-related synapses, while cathodal tDCS should improve it due to a superior strengthening of the relevant synaptic connections. To unravel also small effects of homeostatic plasticity on performance, if present, we added a further experimental arm, in which D-Cycloserine (CYC) was added. CYC is a partial NMDA receptor-agonist, which, if administered in a low dosage, agonists selectively already active NMDA receptors (Thomas et al. 1988). Thus when combined with cathodal tDCS, it should selectively enhance the activity of learning-related synapses, if homeostatic rules apply. Conversely, when combined with anodal tDCS, it should further reduce the strengthening of learning-related synapses. To prove for the specificity of the effects for motor learning, we added a control experiment, where only random sequences were performed in the cognitive task (Simple reaction time task (RTT).

\section{Materials and Methods}

\section{Subjects}

80 healthy subjects were studied with written informed consent and ethics committee approval (24 subjects each for anodal (12 females, age 23.8 years +/- 2.3 SD) or cathodal tDCS (15 females, age 24.5 years +/- 1.9) in the SRTT experiment, 16 subjects for each tDCS condition in the RTT(anodal tDCS group: 12 females, age 24.8 years +/- 5.3; cathodal tDCS group: 10 females, age 23.2 +/- 2.6 years). Studies were performed by neurologists familiar with emergency situations in a room with life-support equipment. Each subject participated in one stimulation polarity condition only. Thus, a repeated measures design was performed separately for each stimulation polarity. All subjects received anodal or cathodal, and placebo tDCS stimulation with or without medication in different sessions separated by at least one week to prevent carry-over effects of task learning, stimulation and CYC medication. The order of the different stimulation conditions was randomized between subjects. Right- and left-handed subjects were randomized between the groups. 


\section{Transcranial direct current stimulation}

Current (one $\mathrm{mA}$ ) was induced through saline-soaked sponge electrodes (surface $35 \mathrm{~cm}^{2}$ ). tDCS was delivered by a specially developed, battery-driven constant-current stimulator (Schneider Electronic, Gleichen, Germany). Constant current flow was controlled by an amperemeter. tDCS was delivered immediately before the motor learning experiment for 10 min. The stimulating electrode (to which the terms anodal and cathodal tDCS refer to) was placed contralaterally to the performing right hand, the reference electrode ipsilaterally. For stimulation of the primary motor cortex, the stimulating electrode was placed above C3 (international 10-20 system) and the reference electrode above the contralateral orbita. tDCS was started in a ramp-like fashion by increasing current strength gradually for the first and last 10 seconds of tDCS. For placebo tDCS, current was switched off after 30 seconds of stimulation. This protocol has been demonstrated to reliably blind subjects with regard to the stimulation condition (Gandiga et al. 2006).

\section{Pharmacological Interventions}

$100 \mathrm{mg}$ CYC or equivalent placebo (PLC) drugs were administered to the subjects orally two hours before the start of each experimental session, Two hours after oral intake, CYC induces a stable plasma level (van Berckel et al. 1997) and alters the efficacy of anodal tDCS to enhance motor cortex excitability (Nitsche et al. 2004). To avoid cumulative drug effects, each experimental session was separated by at least one week. Subjects were blinded to the respective pharmacological condition.

\section{Serial reaction time task}

Subjects were seated in front of a computer screen at eye level behind a response pad with four buttons numbered 1-4 and were instructed to push each button with a different finger of the right hand (index finger for button 1, middle finger for button 2, ring finger for button 3 , and little finger for button 4). An asterisk appeared in one of 4 positions that were horizontally spaced on a computer screen and permanently marked by dots. The subjects were instructed to press the key corresponding to the position of the asterisks as fast as possible. After a button was pushed, the go signal disappeared. The next go signal was displayed $500 \mathrm{~ms}$ later. The learning test consisted of 8 blocks of 120 trials. In block 1 and 6 the sequence of asterisks followed a pseudorandom order in that asterisks were presented equally frequently in each position and never in the same position in two subsequent trials. In block 2 to 5 and 7 and 8 , 
the same 12-trial sequence of asterisk positions repeated itself 10 times (abadbcdacbdc). Subjects were not told about the repeating sequence.

\section{Simple reaction time test}

The random sequence control experiment was identical to the SRTT, apart from the fact that no repetitive sequences, but rather a random order of stimuli, was presented in each block.

\section{Experimental course}

2h after CYC or placebo medication intake, subjects were placed on a comfortable chair in front of a computer monitor and received anodal, cathodal or placebo tDCS for 10 minutes. Immediately after the end of stimulation, the SRTT or the RTT was conducted as described above. Motor task performance required between 10 and 15 min in each subject, dependent on the length of the break between the blocks chosen by the subjects. For the SRTT, a recall test was performed 2, 4 and $24 \mathrm{~h}$ after the learning session, in which first a random sequence and afterwards 2 blocks of the learned sequence were presented.

Subjects were blinded to drug and tDCS condition and were not informed about a reverberating sequence in the learning experiment. To avoid drug, stimulation or sequence interference effects, a one-week break between each session was obligatory. After the last session, subjects were asked if they had noticed any recurring motor sequence throughout the experiments.

\section{Data analysis}

\section{SRTT}

In each trial, response time (RT) was measured from the appearance of the go signal until the first button was pushed by the subject. For each block of trials of a given experimental condition, mean RT was calculated for each subject separately, incorrect responses and reaction times of less than $200 \mathrm{~ms}$ or more than $3000 \mathrm{~ms}$ or those that were above 3 standard deviations of the individual subject's mean response time were discarded. Mean reaction times were standardized to block 1 for each subject in each stimulation condition separately. Furthermore, the standard deviation of reaction times for each subject in every block was calculated as an index of variability of reaction times. An error rate (ER) was calculated to assess the number of incorrect responses for each block and each subject in each stimulation condition. Statistical analyses were performed via ANOVA (level of significance 0.05, independent variables tDCS (anodal, cathodal, sham stimulation), drug (CYC or placebo medication), and block for RT, ER, and variability for absolute and standardized (RT only) 
values. Additionally, RT, ER, and variability value differences between the respective tDCS/drug-conditions were compared by repeated measures two-tailed Students `t-tests (level of significance 0.05 ) within each block of the task for a given stimulation polarity and drug condition. Since the RT and ER differences between block 5, and 6 are thought to represent an exclusive measure of implicit learning, interactive Student's t-tests (Cohen, 1977) were performed to compare the respective differences for the anodal/cathodal/CYC stimulation conditions on the one hand, and the non-current/placebo medication condition on the other (SRTT only).

To rule out stimulation-independent baseline reaction-time differences between the tDCSgroups of a given tDCS/drug-condition, the respective absolute reaction times of block one were compared by Student's t-tests $(\mathrm{p}<0.05)$.

$R T T$

For the random sequence control experiment, repeated measures ANOVAs (independent variables: tDCS (anodal, cathodal, sham stimulation), drug (CYC or placebo medication, and block) and post hoc t-tests were calculated for absolute RT, ER and variability. Critical pvalues were set to 0.05 for all tests.

\section{Results}

SRTT

With regard to absolute reaction time, the ANOVA revealed a significant main effect of block and significant interactions between block x medication, block x tDCS polarity and block x tDCS polarity $x$ tDCS vs. sham stimulation (table 1 ). As depicted in figure 1 , this is due to prolonged RT in the anodal tDCS/CYC condition as compared to the sham stimulation/placebo medication condition, primarily in the later blocks. Baseline RT did not differ significantly between the experimental conditions. However, to rule out that slight baseline differences influenced these results, we performed additional calculations for baseline-standardized values. Here the main effects of block and tDCS polarity and the interaction between block $\mathrm{x}$ tDCS polarity $\mathrm{x}$ tDCS vs. sham stimulation were significant (table 1). This is due to slower RT in case of anodal tDCS under CYC as compared to the sham stimulation/placebo medication condition, especially in the later blocks, as well as to slower RT in the cathodal tDCS condition under CYC as compared to the sham stimulation placebo medication condition. For cathodal tDCS under CYC, this slowing of RT seems to be 
specific for sequence learning, since here the interactive $t$-test is significant with regard to block 5 (sequence) and block 6 (random stimuli) (figure 2).

For ER and variability, the ANOVA shows significant main effects of block (ER: $F=11.767$, $\mathrm{p}<0.001$; variability $\mathrm{F}=15.794, \mathrm{p}<0.001$ ), due to a reduced number of errors and less variability during performance in the later blocks, but no significant effect of tDCS or medication or interaction of these variables, i.e. RT is increased for block 5, but not block 6 , after cathodal tDCS under CYC as compared to sham tDCS/placebo medication.

\section{$R T T$}

With regard to the random stimuli reaction time task, for RT the ANOVA revealed a main effect of block, but no main effect of the other variables tested or interactions (table 1). For the group which received cathodal tDCS, a trend for reduced RT in the cathodal group can be identified; however, this could not be reproduced for the anodal tDCS groups (figure 3). For ER, the main effects of block and medication (as well as the interaction between block and polarity and the 5-way interaction between all variables tested were significant (table 1). This is due to a reduction of errors - as compared to the respective tDCS and medication placeboconditions - under CYC medication and combination of cathodal tDCS with CYC (figure 4). However, this effect was significant only for some blocks. The ANOVA performed for variability revealed a significant main effect for block $(F=2.860, p<0.007)$, but no other significances.

\section{Discussion}

Homeostatic plasticity is suggested to control for the amount and direction of neuroplastic cortical network modifications to avoid de-stabilization. The relevance of homeostatic influences on cortical plasticity in humans was so far only confirmed neurophysiologically. Here, we explored the importance of homeostatic plasticity for behavior in healthy humans. The results of our study show only limited homeostatic effects of a preconditioning motor cortex excitability enhancement or reduction on subsequent performance in a serial reaction time task or simple reaction time task. These results put the general behavioral relevance of homeostatic plasticity for motor cortex function, especially learning and memory consolidation, into question. 
Impact of preconditioning excitability modulation on sequential motor learning

The results of our study show that prior excitability-enhancing anodal tDCS slow down reaction time in the SRTT, when combined with the partial NMDA receptor-agonist CYC. As the baseline-standardized data show, this effect is especially prominent in the recall conditions. Similarly, cathodal tDCS prior to motor learning impairs learning and early recall, if combined with CYC, as shown by the standardized data. Hereby this effect seems to be specific for the learned sequence.

On first sight, one might assume that this deleterious effect of the combined pharmacologicaltDCS intervention might be attributed to the impact of CYC on motor learning; however, this is improbable because CYC alone did not influence SRTT performance.

For excitability-enhancing anodal tDCS, it has been shown that adjunctive CYC medication increased the efficacy of stimulation to enhance motor cortical excitability (Nitsche et al. 2004). Thus it seems reasonable to argue that combined anodal tDCS and CYC medication enhances cortical excitability to a larger extent than anodal tDCS or CYC alone and thus reduce motor learning and consolidation due to homeostatic mechanisms, which might not apply with lower-grade excitability enhancements, as delivered by CYC or anodal tDCS alone for this task. Alternatively, the task-independent enhancement of network excitability might have had a de-focusing effect on task-related synaptic strengthening. However, this should have increased ER in this specific stimulation/medication condition, which was clearly not the case.

For the pattern of results achieved by excitability-diminishing cathodal tDCS, homeostatic mechanisms seem not to contribute: If homeostatic mechanisms would apply, the excitability diminution achieved by cathodal tDCS should have improved performance, especially if combined with CYC, which should have selectively enhanced the synaptic strengthening of learning-related neuronal networks further. However, the results do not fit with this hypothesis. One possible explanation might be that cathodal tDCS did not suppress of the activation of "wrong reactions" during learning sufficiently, thus that CYC in this case given that cathodal tDCS decreases the strengthening of learning-related synapses - would strengthen not only correct, but also false reactions. Alternatively, one could argue that our healthy subjects were already performing at an optimum level without medication or stimulation, and thus any intervention would not be able to improve performance. This seems unlikely, since in another study of our group, performance could be improved when tDCS was administered during performance (Nitsche et al. 2003a). 
Impact of preconditioning excitability modulation on simple reaction time task performance

The results of the RTT show that tDCS of either polarity as well as CYC and any combination of these interventions does not modify RT significantly. There is a trend for CYC medication to reduce RT, especially in the cathodal tDCS group, which however is not replicated for the other subject group, and thus might be due to chance. Anyway, ER was significantly reduced in single blocks for anodal tDCS, CYC medication and the combination of cathodal tDCS and CYC relative to sham stimulation/placebo medication. This might be due to a benefit from a general network excitability enhancement, as delivered by anodal tDCS and CYC, or by increasing task-related excitability in a relatively silent cortical network, as delivered by the combination of cathodal tDCS with CYC, which both might have helped to choose the correct key press by increasing flexibility in this task. However, these effects were relatively small.

Clearly, tDCS and CYC did not modify performance in a direction compatible with homeostatic mechanisms.

\section{General remarks}

Taken together, the results of this study are not in general accordance with a major impact of homeostatic plasticity on motor learning and reaction time, as tested in our experiments. The only result compatible with a homeostatic effect is a diminution of motor learning by combination of CYC and excitability-enhancing anodal tDCS. However, motor learning was not improved by homeostatic regulations following cathodal tDCS combined with CYC, which was hypothesized if homeostatic plasticity was functionally relevant. Also for simple reaction times, there is no clear hint that homeostatic effects improved performance. Conversely, here a pharmacologically or tDCS-induced excitability-enhancement was slightly beneficial.

This pattern of results points to the fact that a simple transferring of neurophysiological studies about homeostatic influences on LTP-like plasticity to behavioral mechanisms seems not appropriate. On the neurophysiological level, preconditioning with tDCS determined the direction of plasticity induced by repetitive transcranial stimulation homeostatically (Lang et al. 2004, Siebner et al. 2004). In a previous study, we explored the impact of a preconditioning tDCS on associative plasticity, as induced by paired associative stimulation (PAS), which might be more closely linked to learning processes. Here, excitabilityenhancing tDCS boosted the efficacy of the subsequent PAS protocol, while excitabilitydiminishing tDCS resulted in reversed effects (Nitsche et al., 2007). Clearly the results of that study are discrepant with those of the present study, and both of them didn't show uniform 
results. Thus it might be argued that the plasticity-inducing protocols used in the neurophysiological studies do not mimic learning processes closely enough, and that the regulation of homeostatic mechanisms depends on the characteristics of induced plasticity.

Moreover, the results of the present study show that an excitability modulation prior to performance of a motor task might be less suited to improve motor learning than tDCS administered during learning or motor behavior. In a former study, we applied anodal or cathodal tDCS during the same tasks as in the current study. Here, excitability-enhancing anodal tDCS improved both, motor learning in the SRTT and simple motor behavior in the RTT (Nitsche et al. 2003). The reason for this divergent result might be that during tDCS not only NMDA-receptors, but also calcium channels are modulated, while the after-effects of tDCS should be accomplished by modifications of NMDA-receptors alone (Nitsche et al. 2003c). Since intracellular calcium concentration is important for LTP induction (Canepari et al. 2007), enhanced transmembrane calcium conduction, as probably accomplished during anodal tDCS, might improve learning processes. Therefore, the results of this study are in favour for administering tDCS during, and not before, learning to optimize performance.

CYC, a partial NMDA receptor-agonist, has been promoted as a putative cognitive enhancer in recent years. Indeed this drug has been demonstrated to improve cognition in Alzheimer's patients in some pilot studies (Tsai et al. 1998, 1999). In our study, however, CYC alone in healthy subjects was not effective on motor learning, but deteriorated performance when combined with tDCS. Our results argue against a relevant positive effect of CYC on learning in healthy humans. There might be a slight positive effect on simple motor behavior though, as shown by the reduced error count in the RTT under CYC, but this was relatively discrete. Since CYC as applied in our study was shown to modulate neuroplasticity prominently in healthy subjects before, it is improbable that this minor effect was caused by an insufficient dosage of the drug (Nitsche et al. 2004).

Taken together, the results of the actual study put into question a major role of homeostatic plasticity in improving cognition in healthy human subjects. On the contrary, they are in favour for a dissociation of neurophysiologically induced metaplasticity and behavioral consequences. Since it might be that homeostatic mechanisms differ for specific kinds of neuroplasticity induced, more studies are needed, which should explore this topic systematically. 


\section{References}

Abbott LF, Nelson SB. Synaptic plasticity: taming the beast. Nat Neurosci. 2000 Nov;3 Suppl:1178-83.

Abraham WC, Tate WP. Metaplasticity: a new vista across the field of synaptic plasticity. Prog Neurobiol. 1997 Jul;52(4):303-23.

Bienenstock EL, Cooper LN, Munro PW. Theory for the development of neuron selectivity: orientation specificity and binocular interaction in visual cortex. J Neurosci. 1982 Jan;2(1):3248.

Canepari M, Djurisic M, Zecevic D. Dendritic signals from rat hippocampal CA1 pyramidal neurons during coincident pre- and post-synaptic activity: a combined voltage- and calciumimaging study.J Physiol. 2007 Feb 1; [Epub ahead of print]

Cohen, J. (1977). Statistical power analysis for the social sciences (2nd ed.). New York: Academic Press.

Gandiga PC, Hummel FC, Cohen LG. Transcranial DC stimulation (tDCS): a tool for doubleblind sham-controlled clinical studies in brain stimulation. Clin Neurophysiol. 2006 Apr;117(4):845-50.

Iyer MB, Schleper N, Wassermann EM. Priming stimulation enhances the depressant effect of low-frequency repetitive transcranial magnetic stimulation. J Neurosci. 2003 Nov 26;23(34):10867-72.

Lang N, Siebner HR, Ernst D, Nitsche MA, Paulus W, Lemon RN, Rothwell JC. Preconditioning with transcranial direct current stimulation sensitizes the motor cortex to rapid-rate transcranial magnetic stimulation and controls the direction of after-effects. Biol Psychiatry. 2004 Nov 1;56(9):634-9.

Liebetanz, D, Nitsche, MA, Tergau, F, Paulus, W. Pharmacological approach to synaptic and membrane mechanisms of DC-induced neuroplasticity in man. Brain 2002, 125, 2238-47

Muellbacher, W., Ziemann, U., Wissel, J., Dang, N., Kofler, M., Facchini, S., Boroojerdi, B., Poewe, W. \& Hallett, M. (2002). Early consolidation in human primary motor cortex. Nature, 415, 640-4. 
Nitsche, M.A. \& Paulus, W. (2000). Excitability changes induced in the human motor cortex by weak transcranial direct current stimulation. J Physiol, 527, 633-639.

Nitsche, M.A. \& Paulus, W. (2001). Sustained excitability elevations induced by transcranial DC motor cortex stimulation in humans. Neurology, 57, 1899-1901.

Nitsche, MA, Fricke, K, Henschke, U, Schlitterlau, A, Liebetanz, D, Lang, N, Henning, S, Tergau, F, Paulus, W. Pharmacological modulation of cortical excitability shifts induced by transcranial DC stimulation. J Physiol, 2003c, 553, 293-301.

Nitsche MA, Jaussi W, Liebetanz D, Lang N, Tergau F, Paulus W. Consolidation of externally induced human motor cortical neuroplasticity by d-cycloserine.

Neuropsychopharmacology, 2004, 29, 1573-8

Nitsche, MA, Nitsche, MS, Klein, CC, Tergau, F, Rothwell, JC, Paulus W. Level of action of cathodal DC polarisation induced inhibition of the human motor cortex. Clin Neurophysiol 2003b, 114, 600-604

Nitsche MA, Roth A, Kuo M-F, Fischer A-K, Antal A, Liebetanz D, Lang N, Paulus W, Tergau F. Modulation of associative plasticity by general network excitability in the human motor cortex. J Neurosci., 2007 Apr 4;27(14):3807-12

Nitsche, MA, Schauenburg, A, Lang, N, Liebetanz, D, Exner, C, Paulus, W, Tergau, F. Facilitation of implicit motor learning by weak transcranial direct current stimulation of the primary motor cortex in the human. J Cog Neurosci, 2003a, 15: 619-626

Nissen, M.J. \& Bullemer, P. (1987). Attentional requirements of learning: evidence from performance measures. Cogn Psychol, 19, 1-32.

Rioult-Pedotti MS, Friedman D, Donoghue JP (2000). Learning-induced LTP in neocortex. Science 290: 533-536.

Siebner HR, Lang N, Rizzo V, Nitsche MA, Paulus W, Lemon RN, Rothwell JC. Preconditioning of low-frequency repetitive transcranial magnetic stimulation with transcranial direct current stimulation: evidence for homeostatic plasticity in the human motor cortex. J Neurosci. 2004 Mar 31;24(13):3379-85. 
Stefan K, Wycislo M, Gentner R, Schramm A, Naumann M, Reiners K, Classen J. Temporary occlusion of associative motor cortical plasticity by prior dynamic motor training. Cereb Cortex. 2006 Mar;16(3):376-85

Tsai GE, Falk WE, Gunther J, Coyle JT. Improved cognition in Alzheimer's disease with short-term D-cycloserine treatment. Am J Psychiatry. 1999 Mar;156(3):467-9.

Tsai GE, Falk WE, Gunther J. A preliminary study of D-cycloserine treatment in Alzheimer's disease. J Neuropsychiatry Clin Neurosci. 1998 Spring;10(2):224-6.

Thomas JW, Hood WF, Monahan JB, Contreras PC, O'Donohue TL (1998). Glycine modulation of the phencyclidine binding site in mammalian brain. Brain Res 442: 396-398.

Turrigiano GG, Nelson SB. Hebb and homeostasis in neuronal plasticity. Curr Opin Neurobiol. 2000 Jun;10(3):358-64

van Berckel BN, Lipsch C, Timp S, Gispen-de Wied C, Wynne H, van Ree JM, Kahn RS (1997). Behavioral and neuroendocrine effects of the partial NMDA agonist D-cycloserine in healthy subjects. Neuropsychopharmacology 16: 317-324.

Ziemann U, Ilic TV, Pauli C, Meintzschel F, Ruge D. Learning modifies subsequent induction of long-term potentiation-like and long-term depression-like plasticity in human motor cortex. J Neurosci. 2004 Feb 18;24(7):1666-72 


\section{Table 1: Results of the ANOVAs performed for the SRTT and RTT experiment}

For the SRTT (reaction time, absolute values), the main effect of block and some interactions are significant. For the baseline-standardized reaction time, additionally the main effect of tDCS polarity is significant. With regard to the RTT, the main effect of block is significant for absolute reaction time and error count. For error count, the interactions between block x tDCS polarity and the 4-way interaction between all independent variables are also significant. The asterisks mark significances (critical p-value 0.05). $\mathrm{df}=$ degrees of freedom, $\mathrm{F}=\mathrm{F}$-value, $\mathrm{p}=$ probability.

\begin{tabular}{|c|c|c|c|}
\hline Experiment 1 (SRTT) absolute reaction time & $\mathrm{df}$ & $\mathrm{F}$ & $p$ \\
\hline Block & 13 & 140.106 & $<0.001^{*}$ \\
\hline tDCS vs sham & 1 & 1.889 & 0.176 \\
\hline Medication & 1 & 1.372 & 0.247 \\
\hline tDCS polarity & 13 & 0.340 & 0.563 \\
\hline Block $x$ tDCS vs sham & 1 & 1.223 & 0.244 \\
\hline Block $x$ medication & 13 & 1.844 & $0.023^{*}$ \\
\hline Block $x$ tDCS polarity & 13 & 2.294 & $0.003^{*}$ \\
\hline tDCS vs sham $x$ medication & 1 & 0.148 & 0.702 \\
\hline tDCS vs sham $x$ tDCS polarity & 1 & 0.307 & 0.582 \\
\hline Medication $x$ tDCS polarity & 1 & 0.724 & 0.399 \\
\hline Block $x$ tDCS vs sham $x$ medication & 13 & 0.468 & 0.962 \\
\hline Block $x$ tDCS vs sham $x$ tDCS polarity & 13 & 1.814 & $0.026^{*}$ \\
\hline Block $x$ medication $x$ tDCS polarity & 13 & 0.321 & 0.995 \\
\hline tDCS vs sham $x$ medication $x$ tDCS polarity & 1 & 0.001 & 0.973 \\
\hline Block $x$ tDCS vs sham $x$ medication $x$ tDCS polarity & 13 & 0.507 & 0.945 \\
\hline \multicolumn{4}{|l|}{ Experiment 1 (SRTT) standardised reaction time } \\
\hline Block & 13 & 97.854 & $<0.001^{*}$ \\
\hline tDCS vs sham & 1 & 1.815 & 0.184 \\
\hline Medication & 1 & 1.919 & 0.173 \\
\hline tDCS polarity & 13 & 2.841 & $0.001^{*}$ \\
\hline Block $x$ tDCS vs sham & 1 & 0.005 & 0.944 \\
\hline Block $x$ medication & 13 & 1.271 & 0.226 \\
\hline Block $x$ tDCS polarity & 13 & 0.718 & 0.746 \\
\hline tDCS vs sham $x$ medication & 1 & 0.041 & 0.841 \\
\hline tDCS vs sham $x$ tDCS polarity & 1 & 1.190 & 0.281 \\
\hline Medication $x$ tDCS polarity & 1 & 0.009 & 0.923 \\
\hline Block $x$ tDCS vs sham $x$ medication & 13 & 0.733 & 0.731 \\
\hline Block $x$ tDCS vs sham $x$ tDCS polarity & 13 & 2.097 & $0.013^{*}$ \\
\hline Block $x$ medication $x$ tDCS polarity & 13 & 0.647 & 0.815 \\
\hline tDCS vs sham $x$ medication $x$ tDCS polarity & 1 & 0.031 & 0.860 \\
\hline Block $x$ tDCS vs sham $x$ medication $x$ tDCS polarity & 13 & 0.650 & 0.812 \\
\hline \multicolumn{4}{|l|}{ Experiment $2(R T)$ absolute reaction time } \\
\hline Block & 7 & 3.45 & $0.002^{*}$ \\
\hline tDCS vs sham & 1 & 0.009 & 0.926 \\
\hline Medication & 1 & 0.430 & 0.517 \\
\hline
\end{tabular}




\begin{tabular}{|c|c|c|c|}
\hline tDCS polarity & 1 & 1.519 & 0.227 \\
\hline Block $x$ tDCS vs sham & 7 & 1.272 & 0.265 \\
\hline Block $x$ medication & 7 & 0.898 & 0.509 \\
\hline Block $x$ tDCS polarity & 7 & 0.991 & 0.438 \\
\hline tDCS vs sham $x$ medication & 1 & 0.100 & 0.754 \\
\hline tDCS vs sham $x$ tDCS polarity & 1 & 0.438 & 0.513 \\
\hline Medication $\mathrm{x}$ tDCS polarity & 1 & 1.136 & 0.295 \\
\hline Block $x$ tDCS vs sham $x$ medication & 7 & 0.396 & 0.904 \\
\hline Block $x$ tDCS vs sham $x$ tDCS polarity & 7 & 0.845 & 0.551 \\
\hline Block $x$ medication $x$ tDCS polarity & 7 & 1.827 & 0.084 \\
\hline tDCS vs sham $x$ medication $x$ tDCS polarity & 1 & 0.013 & 0.908 \\
\hline Block $x$ tDCS vs sham $x$ medication $x$ tDCS polarity & 7 & 1.049 & 0.398 \\
\hline \multicolumn{4}{|l|}{ Experiment 2 (Reaction time) Errors } \\
\hline Block & 7 & 4.477 & $<0.001^{x}$ \\
\hline tDCS vs sham & 1 & 0.709 & 0.406 \\
\hline Medication & 1 & 5.545 & $0.025^{\star}$ \\
\hline tDCS polarity & 1 & 2.092 & 0.158 \\
\hline Block $x$ tDCS vs sham & 7 & 1.453 & 0.186 \\
\hline Block $\mathrm{x}$ medication & 7 & 1.480 & 0.176 \\
\hline Block $x$ tDCS polarity & 7 & 2.100 & $0.045^{\star}$ \\
\hline tDCS vs sham $x$ medication & 1 & 3.239 & 0.082 \\
\hline tDCS vs sham $x$ tDCS polarity & 1 & 0.054 & 0.818 \\
\hline Medication $x$ tDCS polarity & 1 & 0.085 & 0.773 \\
\hline Block $x$ tDCS vs sham $x$ medication & 7 & 0.725 & 0.651 \\
\hline Block $x$ tDCS vs sham $x$ tDCS polarity & 7 & 0.197 & 0.986 \\
\hline Block $x$ medication $x$ tDCS polarity & 7 & 0.694 & 0.677 \\
\hline tDCS vs sham $x$ medication $x$ tDCS polarity & 1 & 2.834 & 0.103 \\
\hline Block $x$ tDCS vs sham $x$ medication $x$ tDCS polarity & 7 & 2.185 & $0.037^{*}$ \\
\hline
\end{tabular}




\section{Figure 1: absolute reaction time (SRTT)}

Depicted are the mean reaction times (ms) for each medication/tDCS combination during learning (block 1-8) and in the recall conditions performed 2h (blocks 2a-c), 4h (blocks 4a-c) and 24h (blocks 24a-c) after sequence learning. In block 1, 6, 2a, 4a, and 24a random stimuli, and in the remaining blocks the sequence is presented. The results show that subjects are getting faster during learning and recall in each condition, but that the reaction time reduction is significantly less accentuated in the anodal tDCS/CYC condition as compared to the sham tDCS/placebo medication condition, especially for later blocks. Asterisks indicate significant deviations between sham tDCS/placebo medication and the other conditions within the respective blocks (paired, two-tailed t-tests, $\mathrm{p}<0.05$ ). Vertical bars depict standard error of mean (S.E.). 


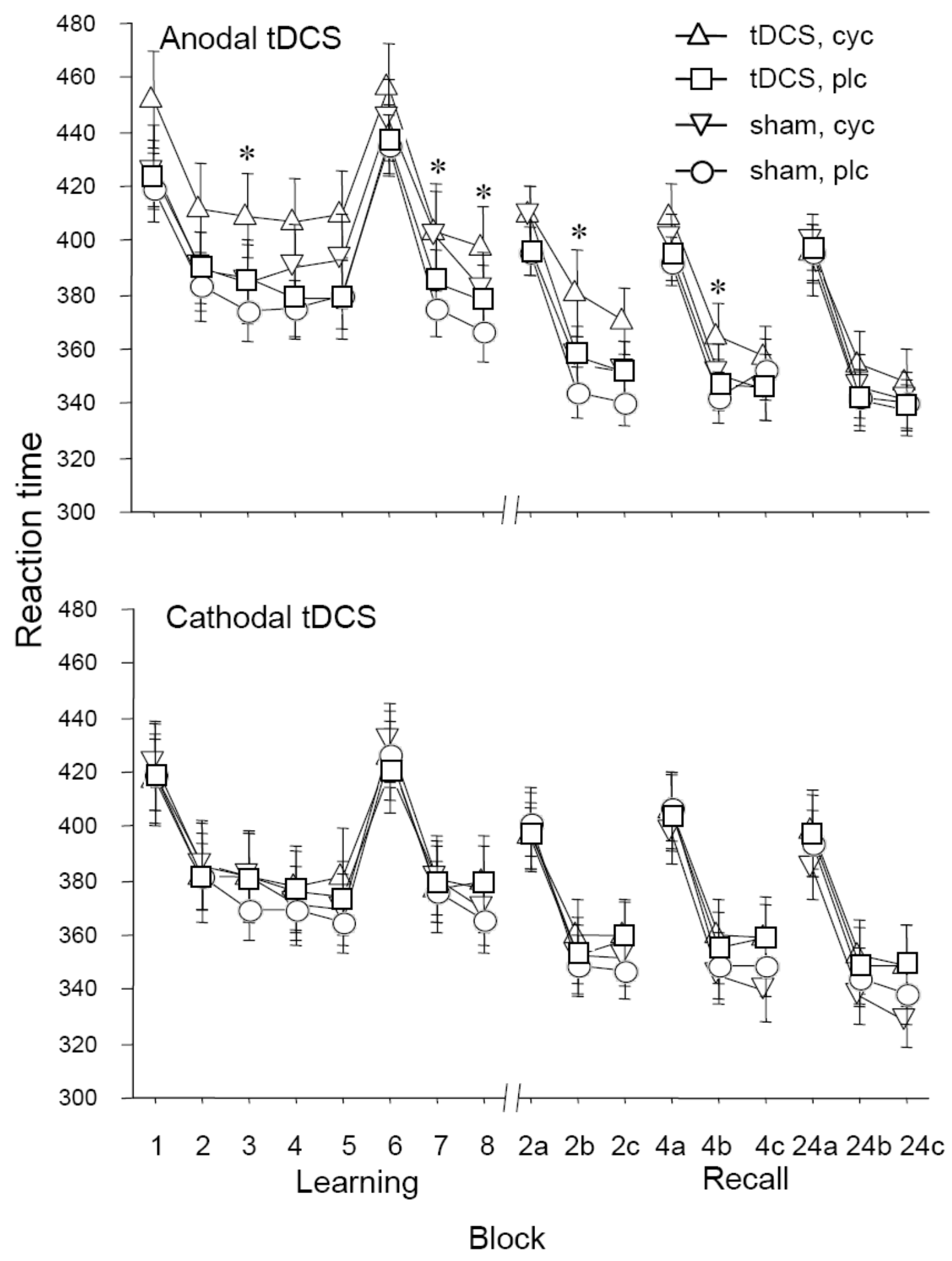




\section{Figure 2: baseline-standardised reaction time (SRTT)}

Depicted are the baseline-standardised mean reaction times for each medication/tDCS combination during learning (block 1-8) and in the recall condtions performed $2 \mathrm{~h}$ (blocks $2 \mathrm{~b}-$ c), 4h (blocks 4b-c) and 24h (blocks 24b-c) after learning. The sequence is displayed in block 2-5, 7-8, 2b-c, 4b-c, and 24b-c. In the remaining blocks, random stimuli are presented. The results show that subjects are getting faster during learning and recall in each condition, but that - similarly to absolute RT - RT reduction is significantly less accentuated in the anodal tDCS/CYC condition as compared to the sham tDCS/placebo medication condition, especially for later blocks. Moreover, also cathodal tDCS compromises learning, when combined with CYC. This effect is specific for sequence learning, because it is not apparent in the random sequence 6. Asterisks indicate significant deviations between sham tDCS/placebo medication and the other conditions within the respective blocks (paired, two-tailed t-tests, $\mathrm{p}<0.05$ ). The hash symbol represents a significant interaction between cathodal tDCS/CYC on the one hand and sham tDCS/placebo stimulation on the other with regard to the sequence block 5 and the random block 6 (interactive t-test, $\mathrm{p}<0.05$ ). Vertical bars depict standard error of mean (S.E.). 


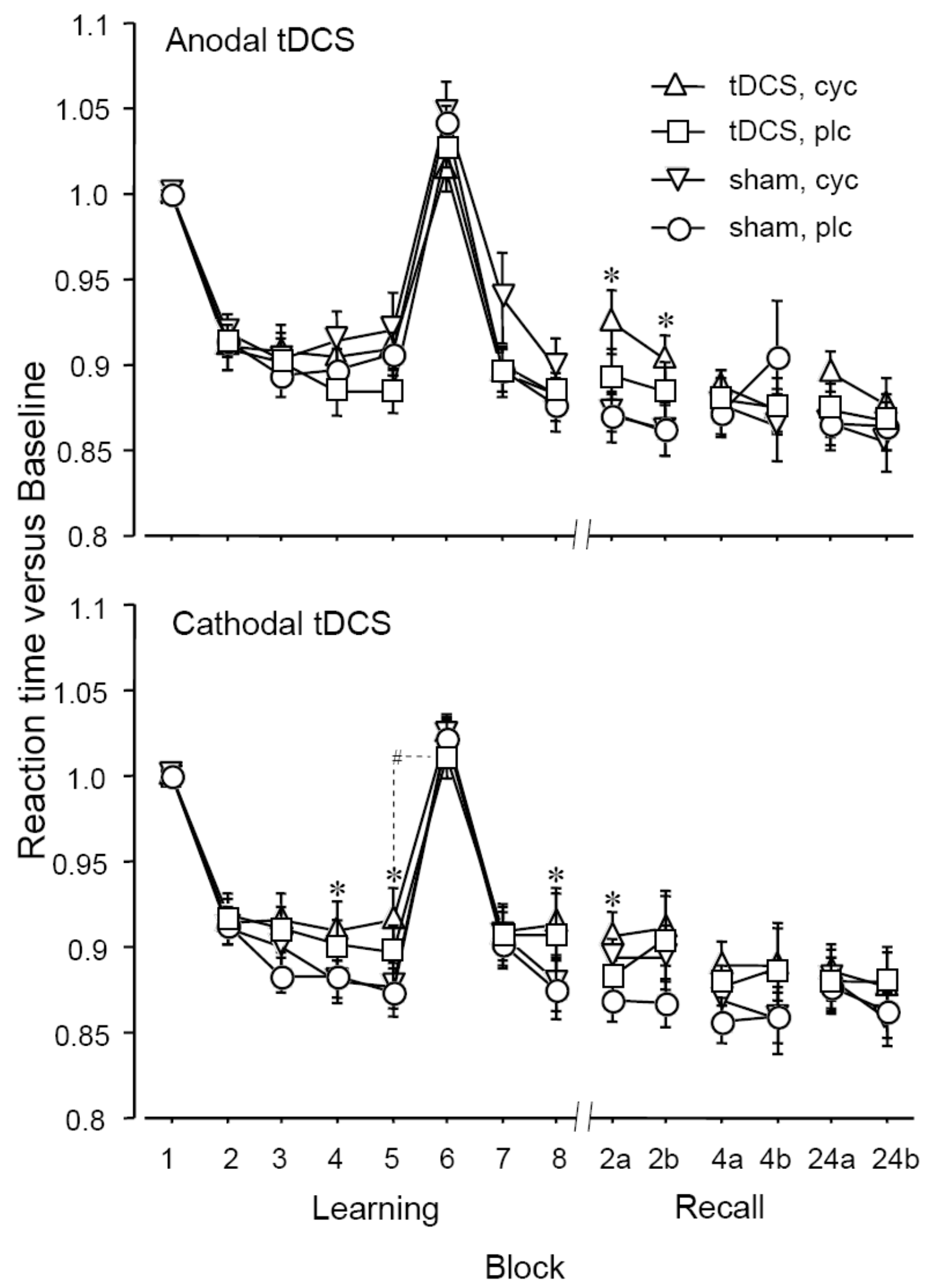




\section{Figure 3: absolute reaction time (RTT)}

Depicted are the mean absolute RTs (ms) for each medication/tDCS combination during performance of the random sequences. The results show no clear trend for a reduction of RT during the course of the experiment, but a non-significant trend for diminished RT under CYC in the cathodal tDCS subjects group. This trend however was not replicated for the anodal tDCS group. Vertical bars depict standard error of mean (S.E.). 


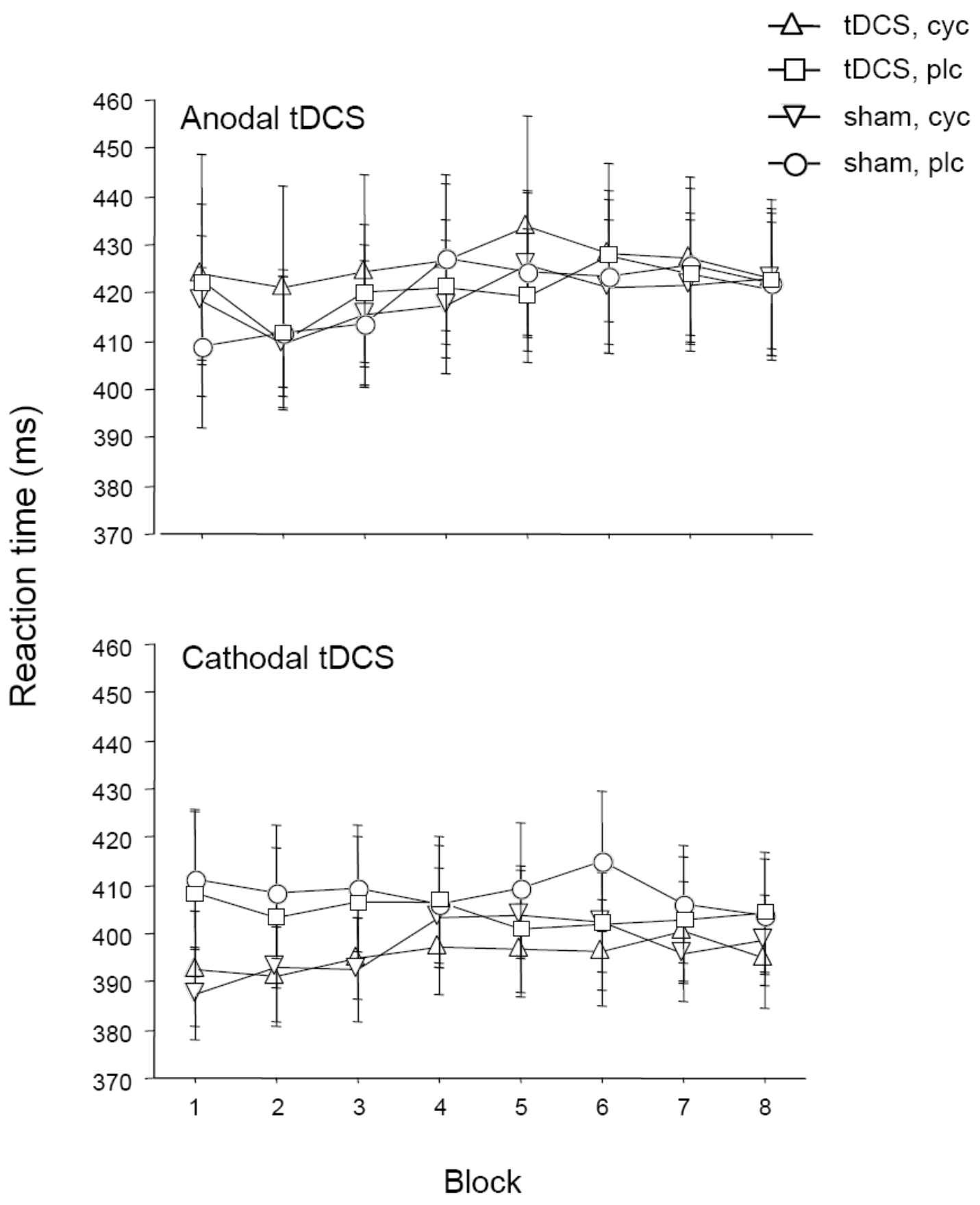




\section{Figure 4: Error counts (RTT)}

Depicted are the mean error counts for each medication/tDCS combination during performance of the random sequences. The results show no clear trend for a reduction of errors during the course of the experiment. However, in the anodal tDCS group CYC and anodal stimulation, but not the combination of both, reduce errors in single blocks, while in the cathodal tDCS group the combination of tDCS and CYC reduces errors in one block. These results are in favour for a slight positive effect of CYC on performance (paired, twotailed t-tests, $\mathrm{p}<0.05)$. Vertical bars depict standard error of mean (S.E.). 


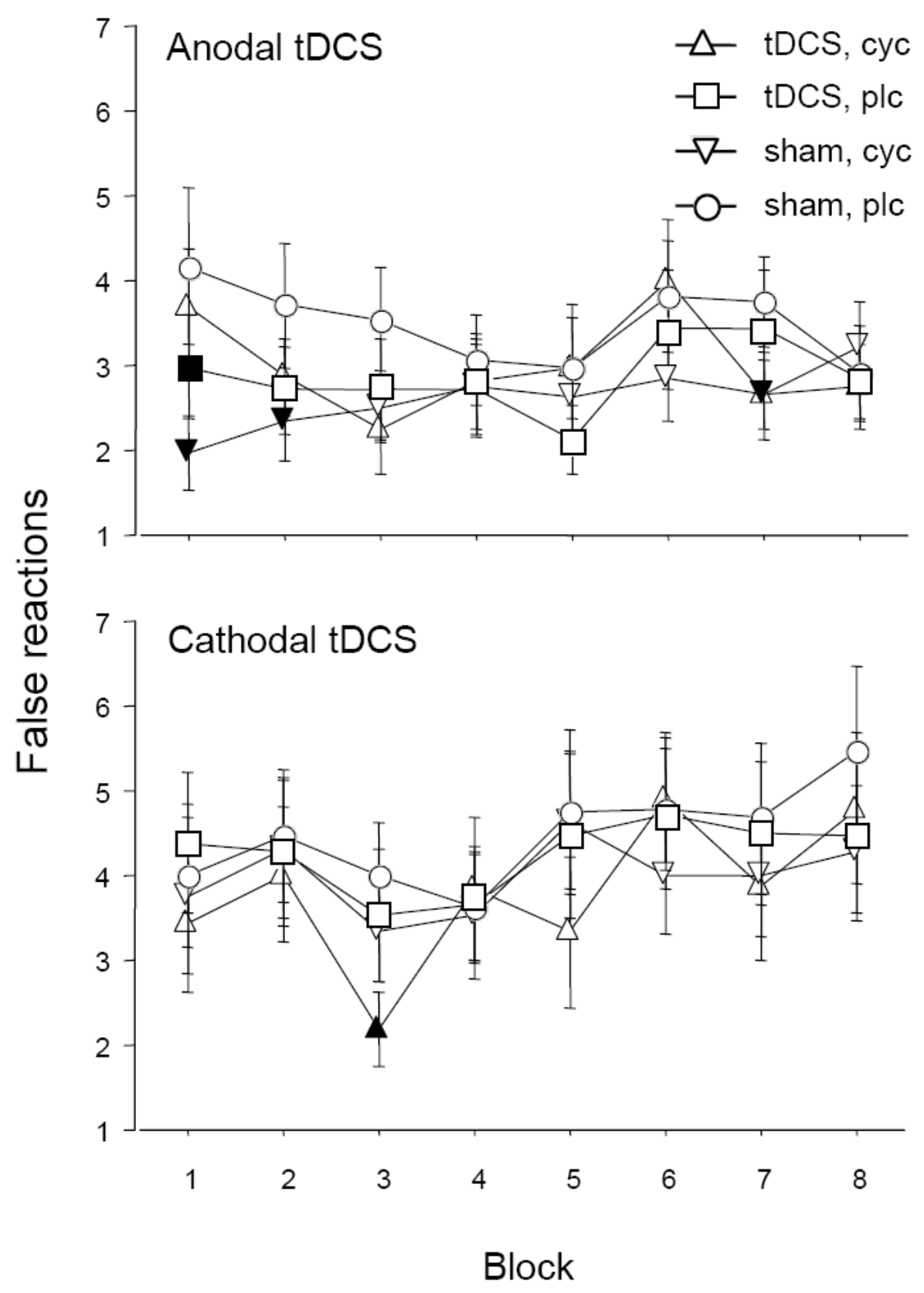




\section{Chapter 3}

\section{Concluding remarks}

The studies included in this thesis address issues which are crucial for the elucidation of the mechanisms underlying neuroplasticity. The distinct evolvement of cortical plastic changes between women and men suggests gender as an intrinsic factor predisposing the sex differences of neurological pathophysiology and cognitive functions associated with neuroplasticity. It also implies the necessity of cautious manipulation and interpretation of plasticity research in the human brain. As revealed by pharmacological interventions, the differential plasticity modulation by DA and ACh, two of the most involved neurotransmitters in the brain's cognitive systems, is in accordance with a focusing mechanism. This might explain the multiple and diverse actions of these neurochemicals in cognitive function within neural networks. The knowledge gained from these experiments is also important for the pharmacotherapeutics of these neurotransmitters in order to preserve the cognitive functions in patients suffering from neuropsychiatric disorders. The last part of this work dealt with homeostatic plasticity, which stablize neuronal networks. Here the modification of global cortical plasticity showed no major influence of homeoplastic regulation in complex motor learning process. It is surprising that the modulation of motor learning at the behavioural level doesn't reflect the direct neurophysiological demonstration of homeoplastic mechanisms by the combination of focal and global neuroplasticity induction protocols. However it also suggests that learning-related neuroplasticity in the motor cortex might be regulated by more tangled modulatory mechanisms in addition to the homeostatic rules, which is of great interest as an object for further studies.

Taken together, an improved knowledge of the mechanisms of neuroplasticity and the awareness of the constrains of its use, both of which we learned from this work, will strengthen the possibility to guide the plastic potential of the brain, and open a broader field of new therapeutic and research perspectives. 


\section{References}

Abbott LF, Nelson SB (2000) Synaptic plasticity: taming the beast. Nat Neurosci 3 Suppl:1178-1183.

Abraham WC (1999) Metaplasticity: Key Element in Memory and Learning? News Physiol Sci 14:85.

Abraham WC, Bear MF (1996) Metaplasticity: the plasticity of synaptic plasticity. Trends Neurosci 19:126-130.

Abraham WC, Tate WP (1997) Metaplasticity: a new vista across the field of synaptic plasticity. Prog Neurobiol 52:303-323.

Antal A, Nitsche MA, Paulus W (2006) Transcranial direct current stimulation and the visual cortex. Brain Res Bull 68:459-463.

Antal A, Nitsche MA, Kincses TZ, Kruse W, Hoffmann KP, Paulus W (2004) Facilitation of visuo-motor learning by transcranial direct current stimulation of the motor and extrastriate visual areas in humans. Eur $\mathrm{J}$ Neurosci 19:2888-2892.

Bindman LJ, Lippold OCJ, Redfearn JWT (1964) The action of brief polarizing currents on the cerebral cortex of the rat (1) during current flow and (2) in the production of long-lasting after-effects. J Physiol (Lond) 172:369-382.

Buonomano DV, Merzenich MM (1998) Cortical plasticity: From synapses to maps. Annual Review Of Neuroscience 21:149-186.

Butefisch CM (2006) Neurobiological bases of rehabilitation. Neurol Sci 27 Suppl 1:S18-23.

Cahill L (2005) His brain, her brain. Sci Am 292:40-47.

Carney MWP (1969) Negative polarisation of the brain in the treatment of manic states. I J Med Sc 2:133-135.

Chen R (2004) Interactions between inhibitory and excitatory circuits in the human motor cortex. Exp Brain Res 154:1-10.

Cooke SF, Bliss TV (2006) Plasticity in the human central nervous system. Brain 129:1659-1673.

Cools R (2006) Dopaminergic modulation of cognitive function-implications for L-DOPA treatment in Parkinson's disease. Neuroscience And Biobehavioral Reviews 30:1-23. 
Costain R, Redfearn JWT, Lippold OCJ (1964) A controlled trial of the therapeutic effects of polarization of the brain in depressive illness. British Journal of Psychiatry 110:786-799.

Dan Y, Poo MM (2004) Spike timing-dependent plasticity of neural circuits. Neuron 44:23-30.

Doyon J, Benali H (2005) Reorganization and plasticity in the adult brain during learning of motor skills. Curr Opin Neurobiol 15:161-167.

Duffau H (2006) Brain plasticity: from pathophysiological mechanisms to therapeutic applications. J Clin Neurosci 13:885-897.

Fitzgerald PB, Fountain S, Daskalakis ZJ (2006) A comprehensive review of the effects of rTMS on motor cortical excitability and inhibition. Clin Neurophysiol 117:2584-2596.

Floel A, Hummel F, Breitenstein C, Knecht S, Cohen LG (2005a) Dopaminergic effects on encoding of a motor memory in chronic stroke. Neurology 65:472-474.

Floel A, Breitenstein C, Hummel F, Celnik P, Gingert C, Sawaki L, Knecht S, Cohen LG (2005b) Dopaminergic influences on formation of a motor memory. Ann Neurol 58:121-130.

Fregni F, Boggio PS, Nitsche M, Bermpohl F, Antal A, Feredoes E, Marcolin MA, Rigonatti SP, Silva MT, Paulus W, Pascual-Leone A (2005) Anodal transcranial direct current stimulation of prefrontal cortex enhances working memory. Exp Brain Res.

Gandolfo F, Li C, Benda BJ, Schioppa CP, Bizzi E (2000) Cortical correlates of learning in monkeys adapting to a new dynamical environment. Proc Natl Acad Sci U S A 97:2259-2263.

Gartside IB (1968) Mechanisms of sustained increases of firing rate of neurones in the rat cerebral cortex after polarization: Role of protein synthesis. Nature 220:383-384.

Gu Q (2002) Neuromodulatory transmitter systems in the cortex and their role in cortical plasticity. Neuroscience 111:815-835.

Hallett M (2001) Plasticity of the human motor cortex and recovery from stroke. Brain Res Brain Res Rev 36:169-174.

Hasselmo ME (2006) The role of acetylcholine in learning and memory. Curr Opin Neurobiol 16:710-715.

Hasselmo ME, Bower JM (1992) Cholinergic suppression specific to intrinsic not afferent fiber synapses in rat piriform (olfactory) cortex. J Neurophysiol 67:1222-1229.

Hasselmo ME, Barkai E (1995) Cholinergic modulation of activity-dependent 
synaptic plasticity in the piriform cortex and associative memory function in a network biophysical simulation. J Neurosci 15:6592-6604.

Hasselmo ME, Giocomo LM (2006) Cholinergic modulation of cortical function. J Mol Neurosci 30:133-135.

Hattori Y, Moriwaki A, Hori Y (1990) Biphasic effects of polarizing current on adenosine-sensitive generation of cyclic AMP in rat cerebral cortex. Neurosci Lett 116:320-324.

Hess G, Donoghue JP (1994) Long-term potentiation of horizontal connections provides a mechanism to reorganize cortical motor maps. J Neurophysiol 71:2543-2547.

Ilic TV, Ziemann U (2005) Exploring motor cortical plasticity using transcranial magnetic stimulation in humans. Ann N Y Acad Sci 1048:175-184.

Islam N, Moriwaki A, Hattori Y, Hayashi Y, Lu YF, Hori Y (1995) c-Fos expression mediated by N-methyl-D-aspartate receptors following anodal polarization in the rat brain. Exp Neurol 133:25-31.

Jay TM (2003) Dopamine: a potential substrate for synaptic plasticity and memory mechanisms. Progress In Neurobiology 69:375-390.

Kimura D (1999) Sex and Cognition. Cambridge, MA: MIT Press.

Kincses TZ, Antal A, Nitsche MA, Bartfai O, Paulus W (2004) Facilitation of probabilistic classification learning by transcranial direct current stimulation of the prefrontal cortex in the human. Neuropsychologia 42:113-117.

Knecht S, Breitenstein C, Bushuven S, Wailke S, Kamping S, Floel A, Zwitserlood P, Ringelstein EB (2004) Levodopa: faster and better word learning in normal humans. Ann Neurol 56:20-26.

Lang N, Siebner HR, Ernst D, Nitsche MA, Paulus W, Lemon RN, Rothwell JC (2004) Preconditioning with transcranial direct current stimulation sensitizes the motor cortex to rapid-rate transcranial magnetic stimulation and controls the direction of after-effects. Biol Psychiatry 56:634-639.

Li CS, Padoa-Schioppa C, Bizzi E (2001) Neuronal correlates of motor performance and motor learning in the primary motor cortex of monkeys adapting to an external force field. Neuron 30:593-607.

Liebetanz D, Nitsche MA, Tergau F, Paulus W (2002) Pharmacological approach to the mechanisms of transcranial DC-stimulation-induced after-effects of human motor cortex excitability. Brain 125:2238-2247.

Lucas-Meunier E, Fossier P, Baux G, Amar M (2003) Cholinergic modulation of the cortical neuronal network. Pflugers Arch 446:17-29.

Malenka RC, Bear MF (2004) LTP and LTD: an embarrassment of riches. Neuron 44:5-21. 
Martin SJ, Grimwood PD, Morris RG (2000) Synaptic plasticity and memory: an evaluation of the hypothesis. Annu Rev Neurosci 23:649-711.

Matsunaga K, Nitsche MA, Tsuji S, Rothwell JC (2004) Effect of transcranial DC sensorimotor cortex stimulation on somatosensory evoked potentials in humans. Clin Neurophysiol 115:456-460.

Muellbacher W, Ziemann U, Wissel J, Dang N, Kofler M, Facchini S, Boroojerdi B, Poewe W, Hallett M (2002) Early consolidation in human primary motor cortex. Nature 415:640-644.

Nitsche MA, Paulus W (2000) Excitability changes induced in the human motor cortex by weak transcranial direct current stimulation. J Physiol $527 \mathrm{Pt}$ 3:633-639.

Nitsche MA, Paulus W (2001) Sustained excitability elevations induced by transcranial DC motor cortex stimulation in humans. Neurology 57:1899-1901.

Nitsche MA, Nitsche MS, Klein CC, Tergau F, Rothwell JC, Paulus W (2003a) Level of action of cathodal DC polarisation induced inhibition of the human motor cortex. Clin Neurophysiol 114:600-604.

Nitsche MA, Liebetanz D, Antal A, Lang N, Tergau F, Paulus W (2003b) Modulation of cortical excitability by weak direct current stimulation--technical, safety and functional aspects. Suppl Clin Neurophysiol 56:255-276.

Nitsche MA, Schauenburg A, Lang N, Liebetanz D, Exner C, Paulus W, Tergau F (2003c) Facilitation of implicit motor learning by weak transcranial direct current stimulation of the primary motor cortex in the human. J Cogn Neurosci 15:619-626.

Nitsche MA, Lampe C, Antal A, Liebetanz D, Lang N, Tergau F, Paulus W (2006) Dopaminergic modulation of long-lasting direct current-induced cortical excitability changes in the human motor cortex. European Journal of Neuroscience 23:1651-1657.

Nitsche MA, Fricke K, Henschke U, Schlitterlau A, Liebetanz D, Lang N, Henning S, Tergau F, Paulus W (2003d) Pharmacological modulation of cortical excitability shifts induced by transcranial direct current stimulation in humans. J Physiol 553:293-301.

Nudo RJ, Wise BM, SiFuentes F, Milliken GW (1996) Neural substrates for the effects of rehabilitative training on motor recovery after ischemic infarct. Science 272:1791-1794.

O'Donnell P (2003) Dopamine gating of forebrain neural ensembles. European Journal Of Neuroscience 17:429-435. 
Pascual-Leone A, Walsh V, Rothwell J (2000) Transcranial magnetic stimulation in cognitive neuroscience--virtual lesion, chronometry, and functional connectivity. Curr Opin Neurobiol 10:232-237.

Pascual-Leone A, Amedi A, Fregni F, Merabet LB (2005) The plastic human brain cortex. Annu Rev Neurosci 28:377-401.

Pascual-Leone A, Davey, N., Rothwell, JC., Wassermann, E., Puri, BK. (2002) Handbook of Transcranial Magnetic Stimulation, 1st Edition. New York: A Hodder Arnold Publication.

Purpura DP, McMurtry JG (1965) Intracellular activities and evoked potential changes during polarization of motor cortex. J Neurophysiol 28:166-185.

Rasmusson DD (2000) The role of acetylcholine in cortical synaptic plasticity. Behav Brain Res 115:205-218.

Rioult-Pedotti MS, Friedman D, Donoghue JP (2000) Learning-induced LTP in neocortex. Science 290:533-536.

Rossini PM, Rossi S (2007) Transcranial magnetic stimulation: diagnostic, therapeutic, and research potential. Neurology 68:484-488.

Rothwell JC (1993) Evoked potentials, magnetic stimulation studies, and event-related potentials. Curr Opin Neurol 6:715-723.

Sanes JN, Donoghue JP (2000) Plasticity and primary motor cortex. Annu Rev Neurosci 23:393-415.

Sarter M, Hasselmo ME, Bruno JP, Givens B (2005) Unraveling the attentional functions of cortical cholinergic inputs: interactions between signal-driven and cognitive modulation of signal detection. Brain Res Brain Res Rev 48:98-111.

Seamans JK, Yang CR (2004) The principal features and mechanisms of dopamine modulation in the prefrontal cortex. Prog Neurobiol 74:1-58.

Siebner HR, Lang N, Rizzo V, Nitsche MA, Paulus W, Lemon RN, Rothwell JC (2004) Preconditioning of low-frequency repetitive transcranial magnetic stimulation with transcranial direct current stimulation: evidence for homeostatic plasticity in the human motor cortex. J Neurosci 24:3379-3385.

Stefan K, Kunesch E, Cohen LG, Benecke R, Classen J (2000) Induction of plasticity in the human motor cortex by paired associative stimulation. Brain 123 Pt 3:572-584.

Stefan K, Kunesch E, Benecke R, Cohen LG, Classen J (2002) Mechanisms of enhancement of human motor cortex excitability induced by interventional paired associative stimulation. J Physiol 543:699-708.

Stefan K, Wycislo M, Gentner R, Schramm A, Naumann M, Reiners K, Classen J (2006) Temporary occlusion of associative motor cortical plasticity by prior 
dynamic motor training. Cerebral Cortex 16:376-385.

Terao Y, Ugawa Y (2002) Basic mechanisms of TMS. J Clin Neurophysiol 19:322-343.

Turrigiano GG, Nelson SB (2000) Hebb and homeostasis in neuronal plasticity. Curr Opin Neurobiol 10:358-364.

Wolters A, Sandbrink F, Schlottmann A, Kunesch E, Stefan K, Cohen LG, Benecke R, Classen J (2003) A temporally asymmetric Hebbian rule governing plasticity in the human motor cortex. J Neurophysiol 89:2339-2345.

Ziemann U, Rothwell JC (2000) I-waves in motor cortex. J Clin Neurophysiol 17:397-405.

Ziemann U, Iliac TV, Pauli C, Meintzschel F, Ruge D (2004) Learning modifies subsequent induction of long-term potentiation-like and long-term depression-like plasticity in human motor cortex. J Neurosci 24:1666-1672. 


\section{Curriculum Vitae}

\section{Min-Fang Kuo}

\section{Education}

03/06-03/07 Research fellow in Sobell Department of Motor Neuroscience and Movement Disorders, Institute of Neurology, UCL, London, UK

Fall, 2004 - PhD program of Center for Systems Neuroscience (ZNV), Göttingen, Germany

Fall, 2004 - Göttingen/London European Graduate School "Neuroplasticity: From Molecules to Systems", Göttingen, Germany

2002-2004 International Graduate Program 'Medical Neurosciences', Charite, Humboldt Universität, Berlin, Germany. MSc. Medical Neurosciences

Master thesis: 'The underlying mechanism of the visual responses induced by transcranial magnetic stimulation'

1995-2002 Department of Medicine, National Cheng Kung University (NCKU), Tainan, Taiwan.

M.D

1999-2001: Clerkship in NCKU Hospital

2001-2002: Internship in NCKU Hospital

Exams : $1^{\text {st }}$ National Examination: July, 1999

$2^{\text {nd }}$ National Examination: July, 2002

$\rightarrow$ Medical Doctor, license to practice medical

\section{$\underline{\text { Publications }}$}

Kuo MF, Unger M, Antal A, Liebetanz D, Lang N, Tergau F, Paulus W, Nitsche 
MA. No Major influence of homeostatic plasticity in complex motor learning in humans. (in submission)

Kuo MF, Fregni F, Paulus W, Nitsche MA. Focusing effect of acetylcholine on neuroplasticity in the human motor cortex. (in submission)

Kuo MF, Paulus W, Nitsche MA. Boosting focally-induced brain plasticity by dopamine. Cerebral Cortex (in press)

Nitsche MA, Roth A, Kuo MF, Fischer AK, Liebetanz D, Lang N, Tergau F, Paulus W. Timing-dependent modulation of associative plasticity by general network excitability in the human motor cortex. J. Neurosci. 2007 Apr 4;27(14):3807-12

Kuo MF, Paulus W, Nitsche MA. Sex differences in cortical neuroplasticity in humans. Neuroreport. 2006 Nov 6;17(16):1703-7

\section{Conference Activities, Presentations}

May 2007 Dopamine 50 years’ congress (Göteborg, Sweden)

Boosting synapse-specific brain functions by dopamine. MF Kuo, W

Paulus, MA Nitsche. (poster)

March $2007 \quad 7^{\text {th }}$ Meeting of the German Neuroscience Society

- 31st Göttingen Neurobiology Conference (Göttingen, Germany)

Homeostatic plasticity in human motor cortex: a study combining transcranial direct current stimulation and paired associative stimulation.

MF Kuo, A Roth, AK Fischer, D Liebetanz, N Lang, F Tergau, W

Paulus, MA Nitsche. (poster)

October $2006 \quad 10^{\text {th }}$ International Congress of Parkinson's Disease and Movement Disorders (Kyoto, Japan)

Neurophysiological evidence for a dopaminergic focusing effect on human cortical network plasticity. MF Kuo, W Paulus, MA Nitsche. (poster)

July $2006 \quad 5^{\text {th }}$ Forum of European Neuroscience (Vienna, Austria)

Dopaminergic modulation of neuroplasticity in human motor cortex: a study with transcranial direct current stimulation. MF Kuo, W Paulus,

MA Nitsche. (poster)

March $2006 \quad 50^{\text {th }}$ Annual meeting of German Society for Clinical

Neurophysiology (Bad Neuheim, Germany)

Gender differences of cortical neuroplasticity in humans, as revealed by 
transcranial direct current stimulation. MF Kuo, W Paulus, MA Nitsche. (oral presentation)

September 2005 Summer School of European Graduate School "Neuroplasticity: From Molecules to Systems" (London, UK)

Cholinergic modulation of motor cortical plasticity induced by transcranial direct current stimulation. MF Kuo, MA Nitsche, W Paulus. (poster)

Göttingen 29.05.2007 
